Article

\title{
Synthesis of Bis(1,2,3-Triazole) Functionalized Quinoline-2,4-Diones ${ }^{\dagger}$
}

\author{
David Milićević ${ }^{1}$, Roman Kimmel ${ }^{1}$, Martin Gazvoda ${ }^{2}$, Damijana Urankar ${ }^{2}$, Stanislav Kafka ${ }^{1, *}$ \\ and Janez Košmrlj 2,*iD \\ 1 Department of Chemistry, Faculty of Technology, Tomas Bata University in Zlin, 76001 Zlin, Czech Republic; \\ david.milicevic@gmail.com (D.M.); r.kimmel@centrum.cz (R.K.) \\ 2 Faculty of Chemistry and Chemical Technology, University of Ljubljana, SI-1000 Ljubljana, Slovenia; \\ martin.gazvoda@fkkt.uni-lj.si (M.G.); damijana.urankar@fkkt.uni-lj.si (D.U.) \\ * Correspondence: kafka@utb.cz (S.K.); janez.kosmrlj@fkkt.uni-lj.si (J.K.); \\ Tel.: +420-57-603-1115 (S.K.); +386-1-479-8558 (J.K.) \\ + Dedicated to Professor Oldřich Paleta on his 80th birthday.
}

Received: 25 June 2018; Accepted: 4 September 2018; Published: 10 September 2018

check for updates

\begin{abstract}
Derivatives of 3-(1H-1,2,3-triazol-1-yl)quinoline-2,4(1H,3H)-dione unsubstituted on quinolone nitrogen atom, which are available by the previously described four step synthesis starting from aniline, were exploited as intermediates in obtaining the title compounds. The procedure involves the introduction of propargyl group onto the quinolone nitrogen atom of mentioned intermediates by the reaction of them with propargyl bromide in $N, N$-dimethylformamide (DMF) in presence of a potassium carbonate and the subsequent formation of a second triazole ring by copper catalyzed cyclisation reaction with azido compounds. The products were characterized by ${ }^{1} \mathrm{H},{ }^{13} \mathrm{C}$ and ${ }^{15} \mathrm{~N}$ NMR spectroscopy. The corresponding resonances were assigned on the basis of the standard $1 \mathrm{D}$ and gradient selected $2 \mathrm{D}$ NMR experiments $\left({ }^{1} \mathrm{H}-{ }^{1} \mathrm{H}\right.$ gs-COSY, ${ }^{1} \mathrm{H}-{ }^{13} \mathrm{C}$ gs-HSQC, ${ }^{1} \mathrm{H}-{ }^{13} \mathrm{C}$ gs-HMBC) with ${ }^{1} \mathrm{H}-{ }^{15} \mathrm{~N}$ gs-HMBC as a practical tool to determine ${ }^{15} \mathrm{~N}$ NMR chemical shifts at the natural abundance level of ${ }^{15} \mathrm{~N}$ isotope.
\end{abstract}

Keywords: click chemistry; azido group; quinoline-2,4(1H,3H)-diones; propargyl group; bis(1,2,3-triazole)

\section{Introduction}

The 1,4-disubstituted-1,2,3-triazole heterocyclic motif has become an exceedingly popular structure finding applications in a broad range of areas including materials, biomaterials, metallopharmaceuticals, supramolecular chemistry, chemical sensing and catalysis, to name just a few [1]. In coordination and organometallic chemistry, for example, it became an important ligand scaffold, not only because of simplicity and reliability in its preparation, but also due to a variety of coordination modes offering [2-6]. Owing to the discovery of copper(I)-catalyzed 1,3-cycloaddition of terminal alkynes with organic azides, the CuAAC click reaction, the preparation of 1,4-disubstituted-1,2,3-triazole is facilitated in mild and modular fashion [7,8]. Although this "click triazole" has become a part of a broad range of molecules, its association with quinoline-2,4-diones remains largely underdeveloped. Apart from our recent publication on 3-(1H-1,2,3-triazol-1-yl)quinoline-2,4(1H,3H)-dione derivatives (1, Figure 1) [9], to the best of our knowledge, other 1,2,3-triazole functionalized quinoline-2,4-diones are unprecedented.

As part of our endeavor in quinoline-2,4-dione chemistry [10] as well as functional click triazoles [11,12] and their applications [13], we became interested in the synthesis of bis(1,2,3-triazole) functionalized quinoline-2,4-diones 2 that may potentially serve as functional scaffolds in coordination 
chemistry, molecular sensing and biochemistry. It is noteworthy that many compounds with the quinoline-2,4-dione structure were isolated from fungi, bacteria and plants, possessing broad range of interesting biological activities in vitro and in vivo [10]. Herein we report an approach to quinoline-2,4-diones unsymmetrically substituted with two click triazoles, an extensive ${ }^{1} \mathrm{H},{ }^{13} \mathrm{C}$, and ${ }^{15} \mathrm{~N}$ NMR spectral analyses, and a preliminary investigation of their chelating properties towards arene-ruthenium.

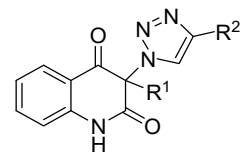

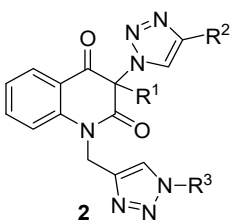

$2 N=N^{\prime}$

Figure 1. A general structure of 1,2,3-triazole quinoline-2,4-diones 1 (left) and the bis(1,2,3-triazole) counterparts 2 (right).

\section{Results and Discussion}

We reasoned that the desired bis(1,2,3-triazole) functionalized quinoline-2,4-diones 2 could be obtained via previously described 3-(1H-1,2,3-triazol-1-yl)quinoline-2,4(1H,3H)-dione derivatives 1 as synthetic intermediates (Scheme 1). The latter were prepared in a four-step reaction sequence starting from aniline, which upon treatment with diethyl 2-methylmalonate and diethyl 2-phenylmalonate initially afforded the corresponding 4-hydroxyquinolin-2(1H)-ones 3a and 3b [14]. Chlorination with sulfuryl chloride into 3-methyl- and 3-phenyl-3-chloroquinolin-2,4(1H,3H)-diones 4a [15] and 4b [16], followed by the nucleophilic displacement of the chlorine atoms with sodium azide, gave 3-methyland 3-phenyl- substituted 3-azidoquinoline-2,4(1H,3H)-diones 5a and 5b [16]. Then we began with copper-catalyzed azide-alkyne cycloaddition reaction (CuAAC).

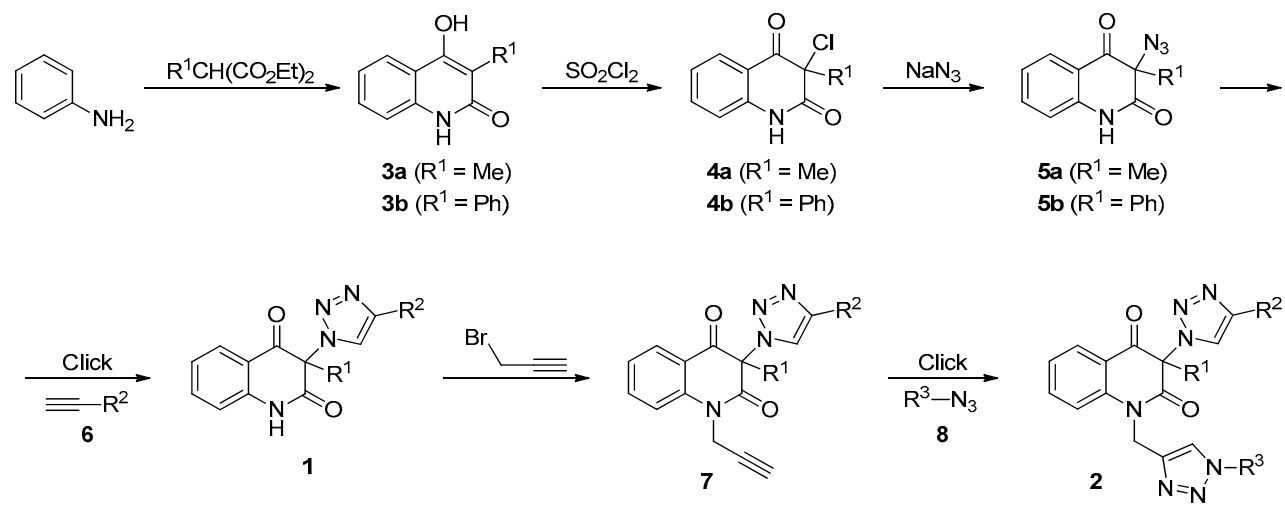

Scheme 1. Preparation of bis(1,2,3-triazole) functionalized quinoline-2,4-diones 2.

Although a large variety of reaction conditions have been developed for the CuAAC reaction [17,18], our previous work in this field has shown that for 3-azidoquinoline-2,4(1H,3H)-diones a combination of copper(II) sulfate pentahydrate and elemental copper $\left(\mathrm{CuSO}_{4} / \mathrm{Cu}^{0}\right)$ in dimethyl sulfoxide (DMSO) provided results that were superior to other combinations. Adopting those previous results in this work some additional optimizations of the reaction conditions were carried out with 5 a and phenylacetylene (6a) as the model substrates. Screening through the reaction solvents indicated that $\mathrm{N}, \mathrm{N}$-dimethylformamide (DMF) is even more efficient than DMSO, providing the desired target compound 1a in shorter reaction times. The influence of the amount of granular copper to the course of the reaction between 5a and equimolar amount of $6 \mathbf{a}$ in DMF was also briefly investigated. While keeping the loading of $\mathrm{CuSO}_{4} \cdot 5 \mathrm{H}_{2} \mathrm{O}$ constant at $10 \mathrm{~mol} \%$ relative to $5 \mathrm{a}$, the amount of the elemental copper was varied from $380 \mathrm{~mol} \%$ to $100 \mathrm{~mol} \%$. The results are summarized in Table 1. 
Table 1. The Effect of Granular Copper to the Conversion of $5 \mathbf{a}^{\text {into }} \mathbf{1} \mathbf{a}^{\mathrm{a}}$.

\begin{tabular}{cccc} 
& $\mathbf{C u}^{\mathbf{0}}$ (mmol) & Reaction Time (h) & Yield $^{\mathbf{b}} \mathbf{( \% )}$ \\
\hline Entry & 3.8 & 0.75 & 98 \\
2 & 3 & 0.75 & 91 \\
3 & 2 & 1 & 89 \\
4 & 1 & 25 & $82^{\mathrm{c}}$ \\
\hline
\end{tabular}

\footnotetext{
a Reaction conditions: $5 \mathbf{a}(1 \mathrm{mmol})$, phenylacetylene $(1 \mathrm{mmol})$, and $\mathrm{CuSO}_{4} \cdot 5 \mathrm{H}_{2} \mathrm{O}(0.1 \mathrm{mmol}), \mathrm{DMF}(4 \mathrm{~mL}), \mathrm{rt}$. The reaction time was determined by thin-layer chromatography (TLC) monitoring of the reaction mixture.

${ }^{b}$ Refers to the yield of isolated pure product. ${ }^{c}$ Complete consumption of $\mathbf{5 a}$ was not reached.
}

Based on the above, in a general procedure, a mixture of 3-azidoquinoline-2,4 $(1 \mathrm{H}, 3 \mathrm{H})$-dione $(5,1.0 \mathrm{mmol})$, a slight excess of terminal alkyne $6(1.05 \mathrm{mmol}), \mathrm{CuSO}_{4} \cdot 5 \mathrm{H}_{2} \mathrm{O}(0.12 \mathrm{mmol})$, and granular copper $(2.0 \mathrm{mmol})$ in DMF $(2.3 \mathrm{~mL})$ was stirred at room temperature, in the presence of air. In addition to phenylacetylene (6a), propargyl alcohol $(\mathbf{6 b})$ was selected as the acetylene partner. The reactions were completed within $30 \mathrm{~min}$. As indicated in Table 2, the products 1 were obtained in excellent yields. By using a more standard $\mathrm{CuSO}_{4} \cdot 5 \mathrm{H}_{2} \mathrm{O}$ / L-ascorbic acid catalyst in $\mathrm{CH}_{2} \mathrm{Cl}_{2}$ / water biphasic system, the cycloaddition between $\mathbf{5 a}$ and $\mathbf{6 a}$ required substantially longer reaction time $(48 \mathrm{~h})$ to achieve a similar yield of the product $1 \mathrm{a}$ as compared to the above $\mathrm{CuSO}_{4} / \mathrm{Cu}^{0} / \mathrm{DMF}$ conditions (Entries 1 and 2).

Table 2. Preparation of compounds 1.

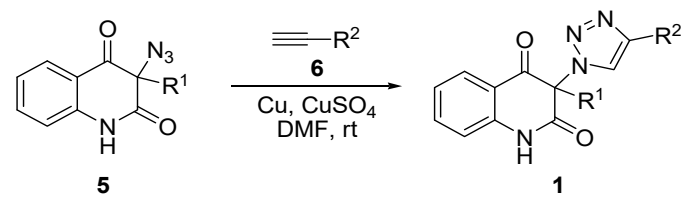

\begin{tabular}{ccccccc}
\hline Entry & Azide $\mathbf{5}$ & $\mathbf{R}^{\mathbf{1}}$ & Acetylene $\mathbf{6}$ & $\mathbf{R}^{\mathbf{2}}$ & Product $\mathbf{1}$ & Yield $^{\mathbf{a}}$ \\
\hline 1 & $\mathbf{5 a}$ & $\mathrm{Me}$ & $\mathbf{6 a}$ & $\mathrm{Ph}$ & $\mathbf{1 a}$ & 95 \\
2 & $\mathbf{5 a}$ & $\mathrm{Me}$ & $\mathbf{6 a}$ & $\mathrm{Ph}$ & $\mathbf{1 a}$ & $83^{\mathrm{b}}$ \\
3 & $\mathbf{5 b}$ & $\mathrm{Ph}$ & $\mathbf{6 a}$ & $\mathrm{Ph}$ & $\mathbf{1 b}$ & 86 \\
4 & $\mathbf{5 a}$ & $\mathrm{Me}$ & $\mathbf{6 b}$ & $\mathrm{CH}_{2} \mathrm{OH}$ & $\mathbf{1 c}$ & 99 \\
5 & $\mathbf{5 b}$ & $\mathrm{Ph}$ & $\mathbf{6 b}$ & $\mathrm{CH}_{2} \mathrm{OH}$ & $\mathbf{1 d}$ & 98 \\
\hline
\end{tabular}

a Refers to percent yield of pure (by TLC and IR) isolated product. ${ }^{\mathrm{b}}$ Employing $\mathrm{CuSO}_{4} \cdot 5 \mathrm{H}_{2} \mathrm{O} / \mathrm{L}$-ascorbic acid $/ \mathrm{CH}_{2} \mathrm{Cl}_{2} /$ water conditions, $48 \mathrm{~h}$ reaction time.

Prior to the introduction of propargyl group at the N1 nitrogen atom of the quinoline-2,4(1H,3H)-dione ring in $\mathbf{1}$, the primary hydroxyl groups at $\mathbf{1} \mathbf{c}$ and $\mathbf{1} \mathbf{d}$ were protected by acetylation by using acetic anhydride in pyridine as shown in Scheme 2. The corresponding acetates 1e and 1 f were obtained in $84-85 \%$ yields.

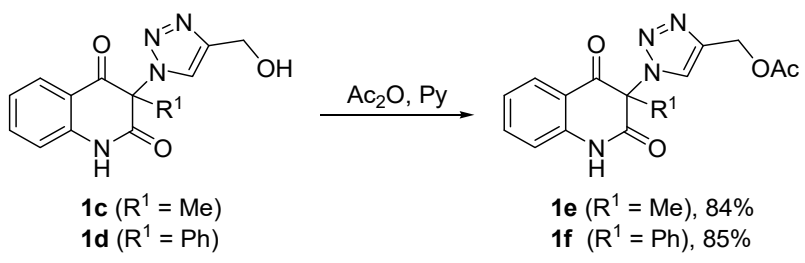

Scheme 2. Preparation of compounds 1e and 1f. 
Alkylation of compounds $\mathbf{1 a}, \mathbf{b}, \mathbf{e}, \mathbf{f}$ with propargyl group was carried out by using 1.5 equivalent of propargyl bromide (6c) and 3 equivalents of potassium carbonate in DMF. These reactions proceeded smoothly within $45 \mathrm{~min}$ at room temperature. The yields are given in Table 3.

Table 3. Preparation of compounds 7.

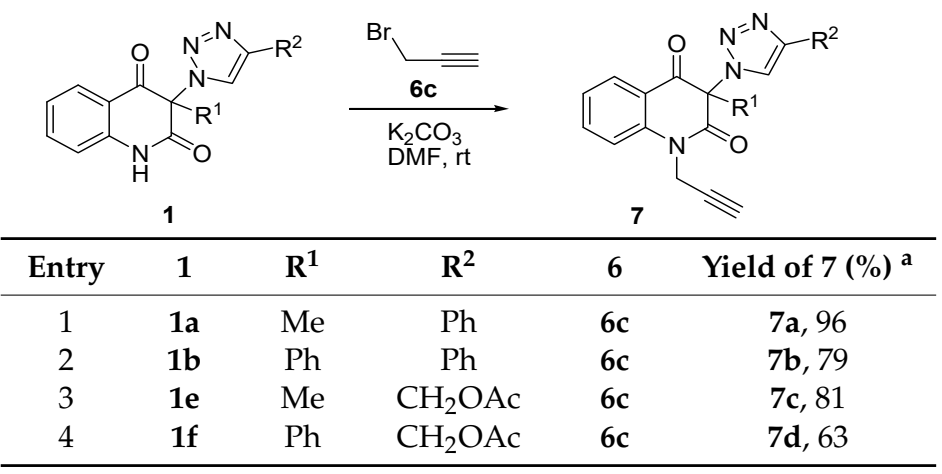

a Refers to percent yield of pure (by TLC and IR) isolated product.

Although $N$-alkylation of the lactam group usually takes place preferentially in quinoline-2,4(1H,3H)-diones [10], the competitive O-alkylation has been documented in similar systems [19]. The N1 position of thus introduced propargyl group in 7 was confirmed by 2D NMR spectroscopy in particular by the presence of the long-range correlations between the propargyl methylene protons and carbon atoms C-8a and C-2 in the ${ }^{1} \mathrm{H}_{-}{ }^{13} \mathrm{C} \mathrm{HMBC}$ spectra (in 7a,c,d) as well as $\mathrm{N} 1$ nitrogen atom in the ${ }^{1} \mathrm{H}-{ }^{15} \mathrm{~N} g s-\mathrm{HMBC}$ spectrum (in 7a).

As the last step of the reaction sequence shown in Scheme 1, monotriazoles 7 were submitted to a second cycloaddition with selected azides $\mathbf{8}$ to give the expected bis-triazoles $\mathbf{2}$. Benzyl azide (8a), azidobenzene $(\mathbf{8 b})$ and tetrazolo[1,5-a]pyridine (8c) were selected as the reaction partners. Whereas benzyl azide (8a) and azidobenzene $(\mathbf{8 b})$ readily reacted into the desired products $\mathbf{2 a}, \mathbf{b}, \mathbf{d}, \mathbf{e}, \mathbf{g}, \mathbf{h}, \mathbf{j}, \mathbf{k}$ at room temperature, tetrazolo[1,5-a]pyridine (8c), a synthetic equivalent for 2-azidopyridine $\left(8 \mathbf{c}^{\prime}\right)$, required harsher reaction conditions (Table 4). This can be explained by the tetrazolyl form in which compound $8 \mathrm{c}$ exists predominantly at room temperature [11]. As the proportion of the azido isomer increases at elevated temperature, the reactions with $8 \mathrm{c}$ were conducted at $100^{\circ} \mathrm{C}$, to afford compounds

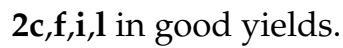

In this case too, some standard click catalyst/solvent combinations were briefly evaluated. The cycloaddition between acetylene $7 \mathrm{c}$ and benzyl azide (8a) with $\mathrm{CuSO}_{4} \cdot 5 \mathrm{H}_{2} \mathrm{O} / \mathrm{Na}$-ascorbate (or L-ascorbic acid) pair in $\mathrm{CH}_{2} \mathrm{Cl}_{2}$ /water and $t$ - $\mathrm{BuOH} /$ water solvent systems required prolonged reaction times, providing lower yields of the product $2 \mathrm{~d}$ as compared to the $\mathrm{CuSO}_{4} / \mathrm{Cu}^{0} / \mathrm{DMF}$ conditions (compare Entries 4-7). In the case of $t-\mathrm{BuOH} /$ water the presence of water in the reaction mixture turned the reactants and products into a gummy material that stuck to the reaction vessel and the magnetic stirring bar, impeding the reaction from going to completion, as already noticed for click reactions with highly hydrophobic reagents [20].

In principle, the "click-propargylation-click" reaction sequence at 3-azidoquinoline-2,4-diones 5 could be altered, providing the target bis-(1,2,3-triazole) functionalized products 2 via bifunctional azidoethynyl quinoline-2,4-dione intermediate 9 as shown in Scheme 3. This would allow orthogonal sequential synthetic strategies for accessing bis(1,2,3-triazole) functionalized materials [21]. We briefly explored this possibility by treating 3-azido-1-propargylquinoline-2,4-dione derivative 9a with phenylacetylene (6a) or benzyl azide (8a) under the above mentioned $\mathrm{CuSO}_{4} / \mathrm{Cu}^{0} / \mathrm{DMF}$ conditions. The corresponding monotriazoles $7 \mathbf{a}(16 \%)$ and 10a (42\%), respectively, were obtained in moderate yields. 
Table 4. Preparation of compounds 2.<smiles>[R]c1cn(C2([R])C(=O)c3ccccc3N(CC#C)C2=O)nn1</smiles>
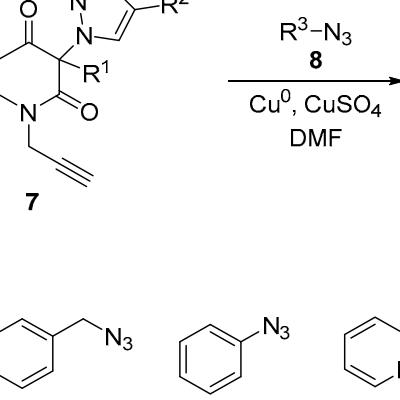

$8 \mathbf{a}$

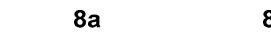

\begin{tabular}{ccc}
\hline Entry & $\mathbf{2}$ & $\mathbf{R}^{\mathbf{1}}$ \\
\hline 1 & $\mathrm{a}$ & $\mathrm{Me}$
\end{tabular}

2 b $\quad \mathrm{Me}$

3 c $\mathrm{Me}$

4 d $\mathrm{Me}$

5 d $\mathrm{Me}$

6 d $\mathrm{Me}$

7 d $\mathrm{Me}$

8 e $\mathrm{Me}$

9 f $\mathrm{Me}$

$10 \quad$ g $\quad \mathrm{Ph}$

11 h $\mathrm{Ph}$

12 i $\mathrm{Ph}$

13

14

15

$\mathrm{Ph}$

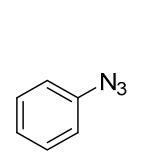

$8 b$
Bn

$\mathrm{Ph}$

2-Py

$\mathrm{CH}_{2} \mathrm{OAc}$
$\mathrm{CH}_{2} \mathrm{OAc}$

$\mathrm{Bn}$

$\mathrm{CH}_{2} \mathrm{OAc}$

$\mathrm{CH}_{2} \mathrm{OAc}$

$\mathrm{CH}_{2} \mathrm{OAc}$

$\mathrm{CH}_{2} \mathrm{OAc}$

Bn

$\mathrm{CH}_{2} \mathrm{OAC}$<smiles>[R]c1cn(C2([R])C(=O)c3ccccc3N(Cc3cn([R])nn3)C2=O)nn1</smiles>

2

$8 c \quad 8 c^{\prime}$

$8 c^{\prime}$

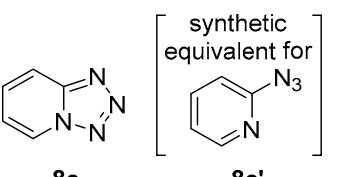

$\begin{array}{ccc}\left({ }^{\circ} \mathrm{C}\right) & \text { Time (h) } & \text { Yield }^{\mathbf{a}}(\mathbf{\%}) \\ 23 & 1 & 97\end{array}$

23

1

99

100

0.5

0.5

23

4

93

Bn 23

96

Bn 23

48

$85^{b}$

Bn 23

$81^{\mathrm{C}}$

Ph 23

$45^{\mathrm{d}}$

-Py 100

$\mathrm{Ph} \quad \mathrm{Bn} \quad 23$

$\mathrm{Ph} \quad \mathrm{Ph} \quad 23$

Ph 2-Py 100

23

Ph 23

2

92

1

85

$1 \quad 92$

194

0.75

2

0.5

0.5

94

57

97

$\mathrm{CH}_{2} \mathrm{OAc} \quad 2-\mathrm{Py} \quad 100$

93

85

a Refers to percent yield of pure (by TLC and IR) isolated product. ${ }^{b}$ Employing $\mathrm{CH}_{2} \mathrm{Cl}_{2} /$ water $/ \mathrm{CuSO}_{4} \cdot 5 \mathrm{H}_{2} \mathrm{O} /$ Na-ascorbate conditions. ${ }^{c}$ Employing $t$ - $\mathrm{BuOH} /$ water $/ \mathrm{CH}_{3} \mathrm{CN} / \mathrm{CuSO} \cdot \cdot 5 \mathrm{H}_{2} \mathrm{O} / \mathrm{Na}$-ascorbate conditions. ${ }^{\mathrm{d}}$ Employing $t$ - $\mathrm{BuOH} /$ water $/ \mathrm{CuSO}_{4} \cdot 5 \mathrm{H}_{2} \mathrm{O} / \mathrm{L}$-ascorbic acid conditions.
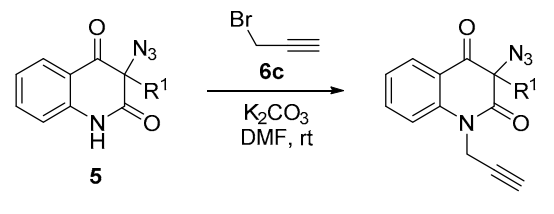

$\left(9 a ; R^{1}=M e, 94 \%\right)$

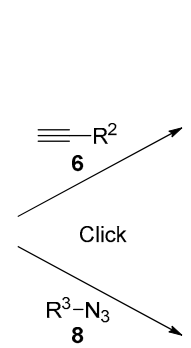

$\frac{\mathrm{R}^{3}-\mathrm{N}_{3}}{8}$

$$
\text { (7a; }
$$

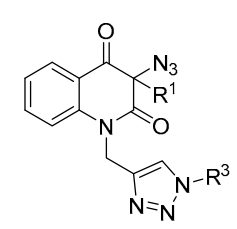

10

$\left(10 a ; R^{1}=M e, R^{3}=B n, 42 \%\right)$
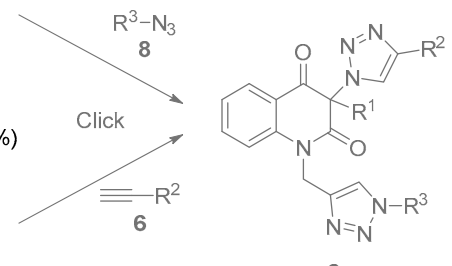

2

Scheme 3. An alternative approach to bis(1,2,3-triazole) functionalized quinoline-2,4-diones 2 through a "propargylation-click-click" reaction sequence.

The compounds $\mathbf{2 a}-\mathbf{1}$ were characterized by ${ }^{1} \mathrm{H},{ }^{13} \mathrm{C}$ and, with the exception of $\mathbf{2 a}, \mathbf{g}, \mathbf{h}$, also by ${ }^{15} \mathrm{~N}$ NMR spectroscopy. The corresponding resonances were assigned on the basis of gradient-selected 2D NMR experiments including ${ }^{1} \mathrm{H}-{ }^{1} \mathrm{H} g s-\mathrm{COSY},{ }^{1} \mathrm{H}-{ }^{13} \mathrm{C} g s-\mathrm{HSQC},{ }^{1} \mathrm{H}-{ }^{13} \mathrm{C} g s-\mathrm{HMBC}$ and ${ }^{1} \mathrm{H}-{ }^{15} \mathrm{~N}$ gs-HMBC. For the atom numbering scheme, see Figure 2. Some characteristic spectral features are discussed below. 

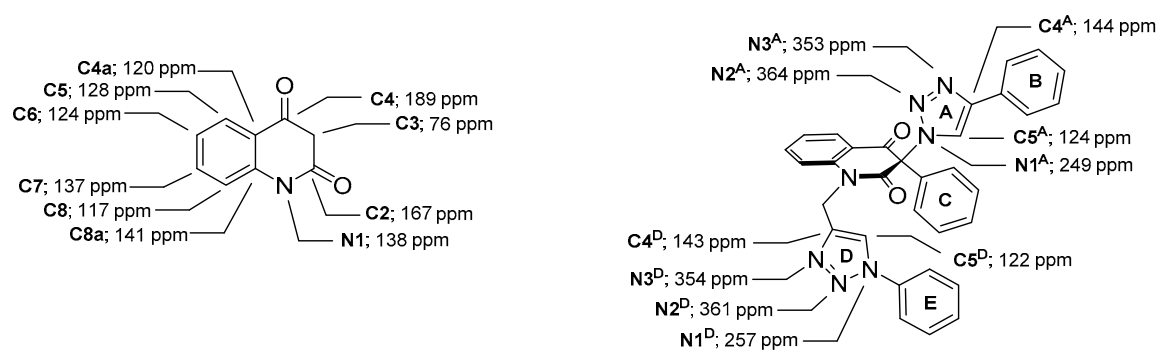

Figure 2. Selected ring and atom numbering along with the chemical shift data (mean values rounded up to whole numbers are provided).

The ${ }^{13} \mathrm{C}$ and ${ }^{15} \mathrm{~N}$ chemical shifts for triazole rings $\mathbf{A}$ and $\mathbf{D}$ (Tables 5 and 6 ) are in a good agreement with those reported previously [11].

To preliminarily assess the applicability of bis-triazole compounds $\mathbf{2}$ as ligands, we decided to examine their coordination abilities to arene-ruthenium. NMR experiment was designed in which compound $\mathbf{2 b}$ and equimolar amount of ruthenium (0.5 equiv of $\left[\mathrm{RuCl}(\mu-\mathrm{Cl})\left(\eta^{6}-p \text {-cymene) }\right]_{2}\right)$ were mixed in $\mathrm{CDCl}_{3}$ in NMR tube at room temperature. $\mathrm{CDCl}_{3}$ was selected as the reaction solvent in place of the coordinative DMSO- $d_{6}$ to avoid possible interference with the metal center (Scheme 4). The reaction mixture was monitored by time dependent ${ }^{1} \mathrm{H}$ NMR spectroscopy indicating an instant change in the resonances for $\mathbf{2} \mathbf{b}$ and $p$-cymene ligands upon mixing to form a new set of resonances that remained unchanged over several days. As shown in Figures 3 and 4, both proton and carbon NMR resonances were severely broadened suggesting the presence of a dynamic process in the solution, presumably an equilibrium with the starting ligand, which can result from a relatively weak ligand-to-metal interaction. Unfortunately, broad NMR resonances prevented an unambiguous structure determination of the product [Ru-Cym]-2b through the 2D NMR techniques due to overlap as well as lack of several indicative crosspeaks in the spectra, especially in ${ }^{1} \mathrm{H}-{ }^{15} \mathrm{~N} g s-\mathrm{HMBC}$. Nevertheless, the analysis of the available NMR data tentatively suggested the coordination of both 1,2,3-triazole rings to the $\mathrm{Ru}-\mathrm{Cym}$ unit as indicated in Scheme 4. Although the coordination properties of the 1,2,3-triazole nitrogen atom N2 are weak, some of us have previously shown that such chelates can be greatly stabilized through an assistance of auxiliary ligand [22].

Table 5. Selected ${ }^{1} \mathrm{H},{ }^{13} \mathrm{C}$ and ${ }^{15} \mathrm{~N}$ NMR chemical shifts in ppm for compounds $\mathbf{1}$ and 7.

\begin{tabular}{|c|c|c|c|c|c|c|c|c|}
\hline & $1 a$ & $1 b$ & 1c & 1d & $7 a$ & $7 \mathrm{~b}$ & $7 c$ & $7 d$ \\
\hline \multicolumn{9}{|c|}{ Quinolone } \\
\hline N1 & - & - & - & - & - & - & 134.4 & - \\
\hline $\mathrm{C} 2$ & 168.5 & 166.8 & 168.7 & 166.8 & 167.7 & 165.8 & 167.8 & 165.8 \\
\hline $\mathrm{C} 3$ & 72.2 & 80.0 & 71.9 & 79.7 & 72.6 & 79.6 & 72.8 & 80.0 \\
\hline $\mathrm{C} 4$ & 190.7 & 188.9 & 190.8 & 189.0 & 189.7 & 187.5 & 189.6 & 187.7 \\
\hline $\mathrm{C} 4 \mathrm{a}$ & 117.4 & 119.2 & 117.5 & 119.2 & 119.0 & 121.0 & 119.2 & 120.9 \\
\hline C5 & 127.7 & 127.6 & 127.6 & 127.5 & 128.2 & 129.2 & 128.0 & 127.8 \\
\hline C6 & 123.5 & 123.5 & 123.3 & 123.4 & 124.2 & 124.6 & 124.0 & 124.2 \\
\hline $\mathrm{C} 7$ & 137.3 & 137.0 & 137.1 & 136.9 & 137.3 & 136.9 & 137.1 & 136.7 \\
\hline C8 & 117.0 & 116.7 & 116.9 & 116.7 & 116.7 & 115.8 & 116.6 & 116.3 \\
\hline $\mathrm{C} 8 \mathrm{a}$ & 141.6 & 140.5 & 141.6 & 140.6 & 140.8 & 140.6 & 140.7 & 140.0 \\
\hline \multicolumn{9}{|c|}{ Ring A } \\
\hline $\mathrm{N} 1^{\mathrm{A}}$ & - & - & - & - & - & - & 247.9 & - \\
\hline $\mathrm{N} 2^{\mathrm{A}}$ & - & - & - & - & - & - & 363.4 & - \\
\hline $\mathrm{N}^{\mathrm{A}}$ & - & - & - & - & - & - & 354.0 & - \\
\hline $\mathrm{C} 4^{\mathrm{A}}$ & 145.8 & 145.3 & 147.4 & 146.8 & 145.9 & 146.0 & 141.5 & 140.9 \\
\hline$C 5^{A}$ & 122.4 & 123.4 & 123.7 & 124.8 & 122.5 & 122.3 & 126.0 & 127.1 \\
\hline $\mathrm{H} 5^{\mathrm{A}}$ & 8.89 & 8.49 & 8.26 & 7.77 & 8.89 & 7.26 & 8.46 & 8.15 \\
\hline
\end{tabular}


Table 6. Selected ${ }^{1} \mathrm{H},{ }^{13} \mathrm{C}$ and ${ }^{15} \mathrm{~N}$ NMR chemical shifts in ppm for compounds 2.

\begin{tabular}{|c|c|c|c|c|c|c|c|c|c|c|c|c|}
\hline & $2 a$ & $2 b$ & $2 c$ & $2 d$ & $2 e$ & $2 f$ & $2 g$ & $2 \mathrm{~h}$ & $2 \mathrm{i}$ & $2 j$ & $2 k$ & 21 \\
\hline \multicolumn{13}{|c|}{ Quinolone } \\
\hline N1 & - & 136.3 & 135.8 & 138.7 & 138.7 & 135.3 & - & - & 137.5 & 140.4 & 140.4 & 138.9 \\
\hline C2 & 168.2 & 168.3 & 168.5 & 168.2 & 168.3 & 168.6 & 166.2 & 166.4 & 166.6 & 166.6 & 166.9 & 166.6 \\
\hline C3 & 72.8 & 73.0 & 73.0 & 71.6 & 71.5 & 73.3 & 80.1 & 80.3 & 80.4 & 79.6 & 79.6 & 79.7 \\
\hline $\mathrm{C} 4$ & 190.0 & 190.0 & 189.9 & 189.4 & 189.4 & 189.9 & 188.2 & 188.2 & 188.1 & 187.9 & 187.9 & 187.9 \\
\hline $\mathrm{C} 4 \mathrm{a}$ & 119.1 & 119.2 & 119.3 & 119.2 & 119.2 & 119.4 & 120.9 & 120.9 & 120.9 & 120.9 & 120.9 & 121.0 \\
\hline C5 & 128.1 & 128.1 & 128.1 & 129.3 & 129.4 & 127.9 & 127.9 & 127.9 & 127.9 & 129.0 & 129.1 & 129.1 \\
\hline C6 & 123.9 & 124.0 & 123.9 & 124.6 & 124.7 & 123.8 & 124.0 & 124.1 & 124.0 & 124.6 & 124.7 & 124.6 \\
\hline C7 & 137.2 & 137.3 & 137.2 & 137.8 & 137.8 & 137.0 & 136.8 & 136.8 & 136.8 & 137.2 & 137.4 & 137.2 \\
\hline $\mathrm{C} 8$ & 116.7 & 116.8 & 116.6 & 116.9 & 116.8 & 116.5 & 116.7 & 116.7 & 116.5 & 116.8 & 116.7 & 116.6 \\
\hline $\mathrm{C} 8 \mathrm{a}$ & 141.5 & 141.6 & 141.4 & 141.7 & 141.7 & 141.3 & 140.8 & 140.7 & 140.5 & 141.1 & 140.9 & 141.2 \\
\hline \multicolumn{13}{|l|}{ Ring A } \\
\hline $\mathrm{N} 1^{\mathrm{A}}$ & - & 248.9 & 248.9 & 248.4 & 248.8 & 247.6 & - & - & 248.7 & 249.8 & 249.9 & 249.7 \\
\hline $\mathrm{N} 2^{\mathrm{A}}$ & - & 363.2 & 363.4 & 361.6 & - & 363.7 & - & - & 367.4 & 365.1 & - & - \\
\hline $\mathrm{N} 3^{\mathrm{A}}$ & - & 347.1 & 347.1 & 355.2 & 355.5 & 353.4 & - & - & 347.2 & 356.9 & 357.2 & 357.1 \\
\hline $\mathrm{C} 4^{\mathrm{A}}$ & 145.9 & 146.0 & 146.0 & 142.3 & 142.3 & 141.6 & 145.4 & 145.4 & 145.4 & 140.9 & 140.9 & 140.9 \\
\hline$C 5^{\mathrm{A}}$ & 122.5 & 122.5 & 122.5 & 124.2 & 124.1 & 126.1 & 123.4 & 123.5 & 123.4 & 126.4 & 126.4 & 126.4 \\
\hline $\mathrm{H} 5^{\mathrm{A}}$ & 8.87 & 8.87 & 8.87 & 7.78 & 7.86 & 8.47 & 8.51 & 8.54 & 8.58 & 7.08 & 7.14 & 7.13 \\
\hline \multicolumn{13}{|c|}{ Ring D } \\
\hline $\mathrm{N} 1^{\mathrm{D}}$ & - & 255.7 & 260.5 & 250.4 & 256.3 & 260.0 & - & - & 260.4 & 250.4 & 256.3 & 261.2 \\
\hline $\mathrm{N} 2^{\mathrm{D}}$ & - & 358.1 & 358.6 & 362.6 & - & 361.9 & - & - & - & 362.9 & - & - \\
\hline$N 3^{\mathrm{D}}$ & - & 353.4 & 356.9 & 350.0 & 351.9 & 356.5 & - & - & 357.7 & 350.5 & 352.9 & 355.8 \\
\hline $\mathrm{C} 4^{\mathrm{D}}$ & 142.2 & 143.3 & 143.2 & 142.9 & 143.2 & 143.2 & 141.9 & 142.9 & 143.0 & 142.9 & 143.2 & 143.0 \\
\hline$C 5^{\mathrm{D}}$ & 123.8 & 121.8 & 120.6 & 123.5 & 121.7 & 120.6 & 124.2 & 122.3 & 120.8 & 123.5 & 121.8 & 121.0 \\
\hline $\mathrm{H} 5^{\mathrm{D}}$ & 8.16 & 8.75 & 8.82 & 7.55 & 8.10 & 8.82 & 8.24 & 8.83 & 8.81 & 7.58 & 8.05 & 8.63 \\
\hline
\end{tabular}

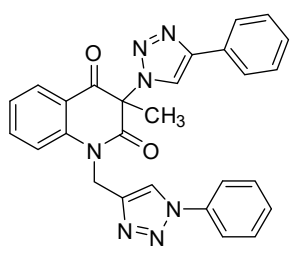

2b

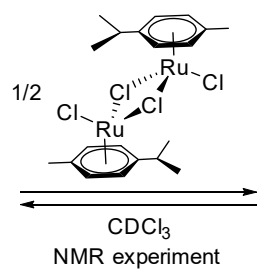

NMR experiment

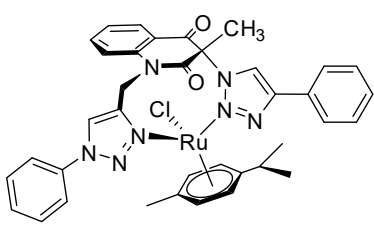

[Ru-Cym]-2b

Scheme 4. Reaction of $\mathbf{2 b}$ with $\left[\mathrm{RuCl}(\mu-\mathrm{Cl})\left(\eta^{6}-p \text {-cymene }\right)\right]_{2}$ with tentatively proposed structure of the [Ru-Cym]-2b complex.

b)

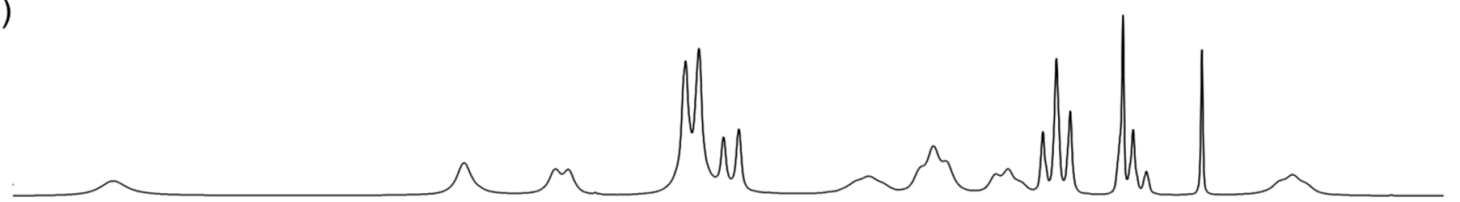

a)

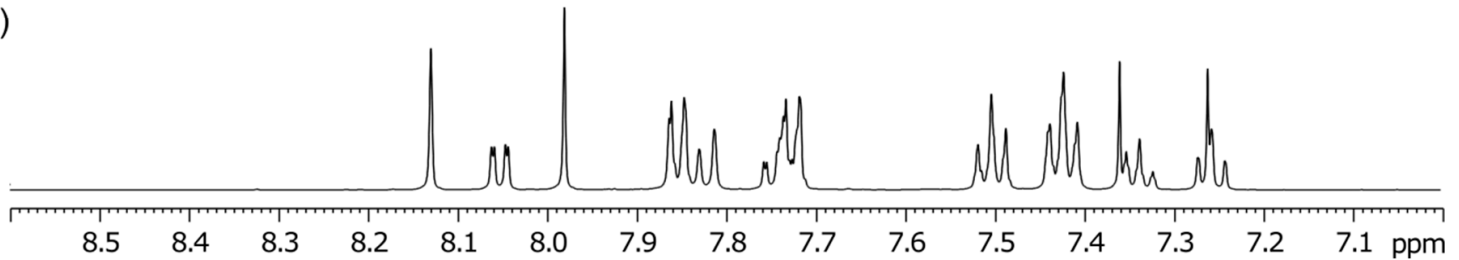

Figure 3. Aromatic region of ${ }^{1} \mathrm{H}$ NMR spectra of: (a) $\mathbf{2} \mathbf{b}$ in $\mathrm{CDCl}_{3}$, and (b) a mixture of $\mathbf{2} \mathbf{b}(42 \mathrm{mM})$ and $\left[\mathrm{RuCl}(\mu-\mathrm{Cl})\left(\eta^{6}-p \text {-cymene }\right)\right]_{2}(21 \mathrm{mM})$ in $\mathrm{CDCl}_{3}$ immediately after dissolution. 
b)

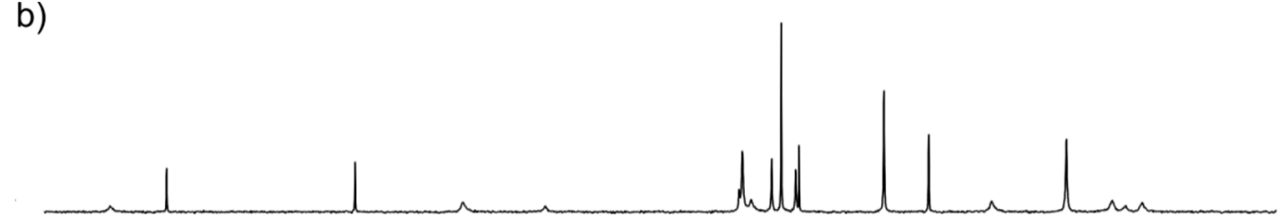

a)

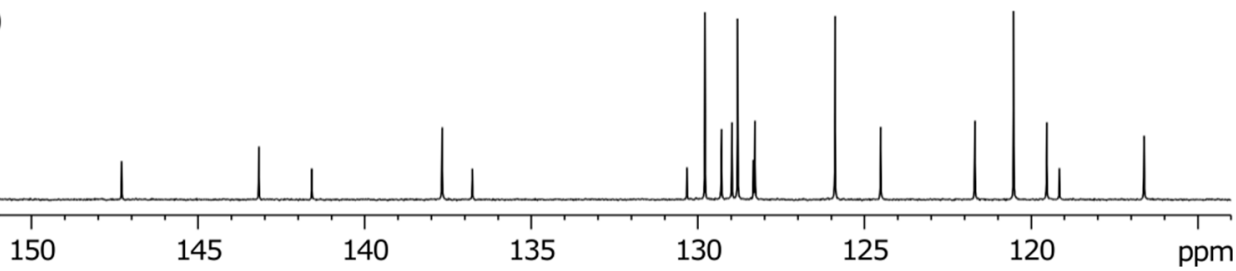

Figure 4. Aromatic region of ${ }^{13} \mathrm{C}$ NMR spectra of: (a) $\mathbf{2} \mathbf{b}$ in $\mathrm{CDCl}_{3}$, and (b) a mixture of $\mathbf{2 b}(42 \mathrm{mM})$ and $\left[\mathrm{RuCl}(\mu-\mathrm{Cl})\left(\eta^{6}-p \text {-cymene }\right)\right]_{2}(21 \mathrm{mM})$ in $\mathrm{CDCl}_{3}$.

Attempts to unambiguously determine the structure of $[\mathrm{Ru}-\mathrm{Cym}]-\mathbf{2 b}$ by variable temperature NMR techniques, as well as to grow crystals suitable for X-ray, failed. All of the above also applies to compounds $2 \mathrm{~g}$ and $\mathbf{2 h}$ that were also preliminarily tested in $[\mathrm{Ru}-\mathrm{Cym}]$ coordination.

\section{Materials and Methods}

\subsection{General Experimental Methods}

The reagents and solvents were used as obtained from the commercial sources. Compounds $\mathbf{3 a}$ [14], $\mathbf{3 b}$ [14], and $\mathbf{5 b}$ [15], as well as benzyl azide (8a) [23], azidobenzene (8b) [11], and tetrazolo[1,5-a]pyridine (8c) [24] were prepared as described in the literature. Column chromatography was carried out on Fluka Silica gel 60 (particle size $0.063-0.2 \mathrm{~mm}$, activity acc. Brockmann and Schodder 2-3). Melting points were determined on the microscope hot stage, Kofler, PolyTherm, manufacturer Helmut Hund GmbH, Wetzlar and are uncorrected. TLC was carried out on pre-coated TLC sheets ALUGRAM ${ }^{\circledR}$ SIL G/UV ${ }_{254}$ for TLC, MACHEREY-NAGEL. NMR spectra were recorded with a Bruker Avance III $500 \mathrm{MHz}$ NMR instrument operating at $500 \mathrm{MHz}\left({ }^{1} \mathrm{H}\right), 126 \mathrm{MHz}$ $\left({ }^{13} \mathrm{C}\right)$ and $51 \mathrm{MHz}\left({ }^{15} \mathrm{~N}\right)$ at $300 \mathrm{~K}$. Proton spectra were referenced to TMS as internal standard, in some cases to the residual signal of DMSO- $d_{5}$ (at $\delta 2.50 \mathrm{ppm}$ ) or $\mathrm{CHCl}_{3}$ (at $\delta 7.26 \mathrm{ppm}$ ). Carbon chemical shifts were determined relative to the ${ }^{13} \mathrm{C}$ signal of DMSO- $d_{6}$ (39.52 ppm) or $\mathrm{CDCl}_{3}(77.16 \mathrm{ppm})$. ${ }^{15} \mathrm{~N}$ chemical shifts were extracted from ${ }^{1} \mathrm{H}_{-}{ }^{15} \mathrm{~N}$ gs-HMBC spectra (with $20 \mathrm{~Hz}$ digital resolution in the indirect dimension and the parameters adjusted for a long-range ${ }^{1} \mathrm{H}-{ }^{15} \mathrm{~N}$ coupling constant of $5 \mathrm{~Hz}$ ) determined with respect to external nitromethane and are corrected to external ammonia by addition of $380.5 \mathrm{ppm}$. Nitrogen chemical shifts are reported to one decimal place as measured of the spectrum, however, the data should not be considered to be more accurate than $\pm 0.5 \mathrm{ppm}$ because of the digital resolution limits of the experiment. Chemical shifts are given on the $\delta$ scale (ppm). Coupling constants $(J)$ are given in Hz. Multiplicities are indicated as follows: s (singlet), d (doublet), t (triplet), q (quartet), $\mathrm{m}$ (multiplet) or br (broadened). Infrared spectra were recorded on FT-IR spectrometer Alpha (Bruker Optik GmbH Ettlingen, Ettlingen, Germany) using samples in potassium bromide disks and only the strongest/structurally most important peaks are listed. Electron impact mass spectra (EI) were recorded on a Shimadzu QP-2010 instrument at $70 \mathrm{eV}$. HRMS spectra were recorded with Agilent 6224 Accurate Mass TOF LC/MS system with electrospray ionization (ESI). Elemental analyses (C, H, N) were performed with FlashEA1112 Automatic Elemental Analyser (Thermo Fisher Scientific Inc., Waltham, MA, USA). 


\subsection{General Procedure for the Synthesis of 3-Chloroquinoline-2,4(1H,3H)-Diones 4 (Scheme 1)}

The 3-Chloroquinoline-2,4(1H,3H)-diones $4 \mathbf{a}$ [15] and $4 \mathbf{b}$ [16], were prepared from 4-hydroxyquinolin-2(1H)-ones $\mathbf{3 a}$ [14] and 3b [14], respectively, according to the procedures described in the literature.

3-Chloro-3-methylquinoline-2,4(1H,3H)-dione (4a). Compound 4a (19.71 g, $94.0 \mathrm{mmol}, 94 \%)$ was prepared from 3a (17.52 g, $100 \mathrm{mmol})$. Yellow crystals, m.p. 178-181 ${ }^{\circ} \mathrm{C}$ (benzene), m.p. [15] 172 ${ }^{\circ} \mathrm{C}$ (acetic acid-water); $R_{\mathrm{f}}=0.52\left(30 \%\right.$ ethyl acetate in chloroform); ${ }^{1} \mathrm{H} \mathrm{NMR}\left(500 \mathrm{MHz}, \mathrm{CDCl}_{3}\right) \delta 1.99(\mathrm{~s}$, $3 \mathrm{H}), 7.06(\mathrm{~d}, 1 \mathrm{H}, J=8.0 \mathrm{~Hz}), 7.22(\mathrm{dd}, 1 \mathrm{H}, J=7.6,7.6 \mathrm{~Hz}), 7.59-7.66(\mathrm{~m}, 1 \mathrm{H}), 8.02(\mathrm{~d}, 1 \mathrm{H}, J=7.7 \mathrm{~Hz})$, $9.41(\mathrm{~s}, 1 \mathrm{H}) ;{ }^{13} \mathrm{C}$ NMR $\left(126 \mathrm{MHz}, \mathrm{CDCl}_{3}\right) \delta 21.2,62.8,116.7,118.1,124.5,129.1,136.8,139.6,169.2,188.4$; IR $\left(\mathrm{cm}^{-1}\right): v$ 3203, 3072, 3004, 2940, 1709, 1674, 1614, 1600, 1486, 1439, 1379, 1239, 770, 440; MS (EI) m/z (\%): $212\left(4,[\mathrm{M}+3]^{+}\right), 211\left(33,\left[\mathrm{M}\left({ }^{37} \mathrm{Cl}\right)\right]^{+}\right), 210\left(17,[\mathrm{M}+1]^{+}\right), 209\left(100,\left[\mathrm{M}\left({ }^{35} \mathrm{Cl}\right)\right]^{+}\right), 208(18), 175(15)$, 174 (36), 146 (68), 128 (17), 120 (18), 119 (59), 92 (32), 91 (15); HRMS (ESI+): $m / z$ calcd for $\mathrm{C}_{10} \mathrm{H}_{9} \mathrm{ClNO}_{2}{ }^{+}$ $[\mathrm{M}+\mathrm{H}]^{+}$210.0316, found 210.0313. Anal. Calcd for $\mathrm{C}_{10} \mathrm{H}_{8} \mathrm{ClNO}_{2}$ (209.63): C, 57.30; H, 3.85; N, 6.68\%. Found: C, 57.18; H, 3.83; N, 6.61\%.

3-Chloro-3-phenylquinoline-2,4(1H,3H)-dione (4b). Compound 4b (26.08 g, $96.0 \mathrm{mmol}, 96 \%)$ was prepared from $3 b(23.73 \mathrm{~g}, 100 \mathrm{mmol})$. Pale yellow needles, m.p. $182-185{ }^{\circ} \mathrm{C}$ (benzene), m.p. [16] $178-180{ }^{\circ} \mathrm{C}$ (ethanol); $R_{\mathrm{f}}=0.57$ (30\% ethyl acetate in chloroform). ${ }^{1} \mathrm{H} \mathrm{NMR}\left(500 \mathrm{MHz}, \mathrm{CDCl}_{3}\right) \delta 7.04$ $(\mathrm{d}, 1 \mathrm{H}, J=8.0 \mathrm{~Hz}, \mathrm{H}-8), 7.18(\mathrm{ddd}, 1 \mathrm{H}, J=7.8,7.4,0.7 \mathrm{~Hz}, \mathrm{H}-6), 7.33-7.39\left(\mathrm{~m}, 3 \mathrm{H}, \mathrm{H}-3^{\mathrm{C}}, \mathrm{H}-4^{\mathrm{C}}, \mathrm{H}-5^{\mathrm{C}}\right)$, 7.51-7.54 (m, 2H, H-2 $\left.{ }^{\mathrm{C}}, \mathrm{H}-6^{\mathrm{C}}\right), 7.55(\mathrm{ddd}, 1 \mathrm{H}, \mathrm{J}=7.3,6.5,1.5 \mathrm{~Hz}, \mathrm{H}-7), 7.97$ (dd, 1H, J = 7.8, 1.2 Hz, H-5), 9.82 (s, 1H, H-1); ${ }^{13} \mathrm{C}$ NMR (126 MHz, $\left.\mathrm{CDCl}_{3}\right) \delta 74.9$ (C-3), 116.9 (C-8), 118.7 (C-4a), 124.7 (C-6),

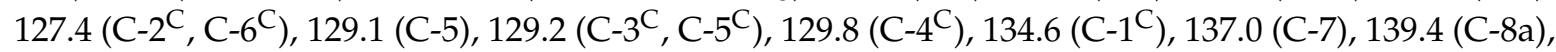
168.8 (C-2), 187.9 (C-4); IR (cm $\left.{ }^{-1}\right): v$ 3201, 3138, 3082, 2992, 2926, 1716, 1680, 1613, 1595, 1485, 1365, 755, 743, 690; MS (EI) m/z (\%): $273\left(7,\left[\mathrm{M}\left({ }^{37} \mathrm{Cl}\right)\right]^{+}\right), 271\left(21,\left[\mathrm{M}\left({ }^{35} \mathrm{Cl}\right)\right]^{+}\right), 238(12), 237(80), 236(100)$, 218 (10), 120 (63), 119 (19), 92 (34), 89 (10), 77 (12), 76 (10), 65 (14), 63 (10); HRMS (ESI+): $m / z$ calcd for $\mathrm{C}_{15} \mathrm{H}_{11} \mathrm{ClNO}_{2}{ }^{+}[\mathrm{M}+\mathrm{H}]^{+}$272.0473, found 272.0480. Anal. Calcd for $\mathrm{C}_{15} \mathrm{H}_{10} \mathrm{ClNO}_{2}$ (271.70): C, 66.31; H, 3.71; N, 5.16\%. Found: C, 66.07; H, 3.62; N, 5.29\%.

\subsection{General Procedure for the Synthesis of 3-Azidoquinoline-2,4(1H,3H)-Diones 5 (Scheme 1)}

To a stirred solution of the 3-chloroquinoline-2,4(1H,3H)-dione 4 (40 mmol) in DMF $(200 \mathrm{~mL})$, sodium azide $(3.90 \mathrm{~g}, 60 \mathrm{mmol})$ was added in small portions during $10 \mathrm{~min}$. The reaction mixture was stirred in darkness for additional $2 \mathrm{~h}$ and then poured into ice-water $(1.5 \mathrm{~L})$. The precipitated solid was filtered, washed with water and dried at $50{ }^{\circ} \mathrm{C}$ in darkness, which afforded product 5 , pure according to TLC and ${ }^{1} \mathrm{H}$ NMR spectrum, which was crystallized from benzene.

3-Azido-3-methylquinoline-2,4(1H,3H)-dione (5a). Compound 5a (8.47 g, $39.2 \mathrm{mmol}, 98 \%)$ was prepared from $4 \mathbf{a}(8.39 \mathrm{~g}, 40.0 \mathrm{mmol})$. Colorless needles, m.p. $158-161{ }^{\circ} \mathrm{C}$ (benzene, $87 \%$ yield of recrystallization); $R_{\mathrm{f}}=0.30$ (30\% ethyl acetate in chloroform). ${ }^{1} \mathrm{H}$ NMR $\left(500 \mathrm{MHz}, \mathrm{CDCl}_{3}\right) \delta 1.86(\mathrm{~s}$, $\left.3 \mathrm{H}, \mathrm{CH}_{3}\right), 7.11(\mathrm{~d}, 1 \mathrm{H}, J=8.0 \mathrm{~Hz}, \mathrm{H}-8), 7.22(\mathrm{dd}, 1 \mathrm{H}, J=7.4,7.4 \mathrm{~Hz}, \mathrm{H}-6), 7.60-7.67$ (m, 1H, H-7), 7.98 (d, $1 \mathrm{H}, J=7.3 \mathrm{~Hz}, \mathrm{H}-5), 9.86(\mathrm{~s}, 1 \mathrm{H}, \mathrm{H}-1) ;{ }^{13} \mathrm{C} \mathrm{NMR}\left(126 \mathrm{MHz}, \mathrm{CDCl}_{3}\right) \delta 23.6\left(\mathrm{CH}_{3}\right), 70.0(\mathrm{C}-3), 116.9(\mathrm{C}-8)$, 118.0 (C-4a), 124.6 (C-6), 128.6 (C-5), 137.2 (C-7), 140.0 (C-8a), 171.6 (C-2), 191.7 (C-4); IR (cm $\left.{ }^{-1}\right): v 3202$, $3078,3005,2936,2108,1708,1682,1614,1598,1485,1392,1284,1156,755,612 ;$ MS (EI) $m / z(\%): 217$ (0.24, $\left.[\mathrm{M}+1]^{+}\right), 216\left(2,[\mathrm{M}]^{+}\right), 147(15), 120(11), 119(100), 92$ (35), 91 (11), 64 (12); HRMS (ESI+): $\mathrm{m} / z$ calcd for $\mathrm{C}_{10} \mathrm{H}_{9} \mathrm{~N}_{4} \mathrm{O}_{2}{ }^{+}[\mathrm{M}+\mathrm{H}]^{+}$217.0720, found 217.0724. Anal. Calcd for $\mathrm{C}_{10} \mathrm{H}_{8} \mathrm{~N}_{4} \mathrm{O}_{2}$ (216.20): C, 55.55; $\mathrm{H}$, 3.73; N, 25.91\%. Found: C, 55.44; H, 3.72; N, 25.98\%.

3-Azido-3-phenylquinoline-2,4(1H,3H)-dione (5b). Compound 5b (10.90 g, $39.2 \mathrm{mmol}, 98 \%)$ was prepared from $4 \mathrm{~b}(10.87 \mathrm{~g}, 40.0 \mathrm{mmol})$. Colorless needles, m.p. $186-189{ }^{\circ} \mathrm{C}$ (benzene, $96 \%$ yield of recrystallization); m.p. [9] 173-181 ${ }^{\circ} \mathrm{C}$ (benzene); $R_{\mathrm{f}}=0.33$ (38\% ethyl acetate in petroleum ether); ${ }^{1} \mathrm{H}$ NMR $\left(500 \mathrm{MHz}, \mathrm{CDCl}_{3}\right) \delta 6.98(\mathrm{~d}, 1 \mathrm{H}, J=8.1 \mathrm{~Hz}, \mathrm{H}-8), 7.16(\mathrm{dd}, 1 \mathrm{H}, J=7.6,7.6 \mathrm{~Hz}, \mathrm{H}-6), 7.38-7.43$ $\left(\mathrm{m}, 3 \mathrm{H}, \mathrm{H}-3^{\mathrm{C}}, \mathrm{H}-4^{\mathrm{C}}, \mathrm{H}-5^{\mathrm{C}}\right), 7.48-7.53\left(\mathrm{~m}, 2 \mathrm{H}, \mathrm{H}-2^{\mathrm{C}}, \mathrm{H}-6^{\mathrm{C}}\right), 7.54(\mathrm{ddd}, 1 \mathrm{H}, J=7.7,7.7,1.6 \mathrm{~Hz}, \mathrm{H}-7)$, 
$7.93(\mathrm{dd}, 1 \mathrm{H}, J=7.8,1.6 \mathrm{~Hz}, \mathrm{H}-5), 9.30(\mathrm{~s}, 1 \mathrm{H}, \mathrm{H}-1) ;{ }^{13} \mathrm{C} \mathrm{NMR}\left(126 \mathrm{MHz}, \mathrm{CDCl}_{3}\right) \delta 78.0(\mathrm{C}-3), 116.7$ (C-8),

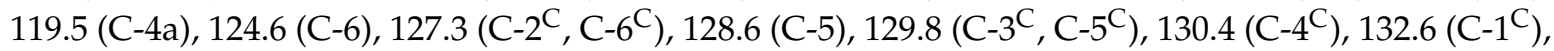
136.9 (C-7), 139.4 (C-8a), 170.2 (C-2), 189.9 (C-4); ${ }^{15} \mathrm{~N}$ NMR (51 MHz, CDCl 3 ) $\delta 133.4(\mathrm{~N}-1)$; IR (cm $\left.{ }^{-1}\right)$ : $v$ 3244, 2105, 1718, 1705, 1685, 1611, 1484, 1356, 1256, 877, 773, 744, 702, 611, 525; MS (EI) $\mathrm{m} / z$ (\%): 250 (7, $\left.\left[\mathrm{M}-\mathrm{N}_{2}\right]^{+}\right), 236\left(8,\left[\mathrm{M}-\mathrm{N}_{3}\right]^{+}\right), 147(28), 120(14), 119$ (100), 104 (15), 92 (32), 77 (10), 76 (10), 64 (14); HRMS (ESI+): $m / z$ calcd for $\mathrm{C}_{15} \mathrm{H}_{11} \mathrm{~N}_{2} \mathrm{O}_{2}{ }^{+}\left[\mathrm{M}-\mathrm{N}_{2}+\mathrm{H}\right]^{+}$251.0815, found 251.0818. HRMS (ESI-): $m / z$ calcd for $\mathrm{C}_{15} \mathrm{H}_{9} \mathrm{~N}_{4} \mathrm{O}_{2}{ }^{-}[\mathrm{M}-\mathrm{H}]^{-}$277.0731, found 277.0732; calcd for $\mathrm{C}_{15} \mathrm{H}_{9} \mathrm{~N}_{2} \mathrm{O}_{2}{ }^{-}\left[\mathrm{M}-\mathrm{N}_{2}-\mathrm{H}\right]^{-}$ 249.0670, found 249.0671. Anal. Calcd for $\mathrm{C}_{15} \mathrm{H}_{10} \mathrm{~N}_{4} \mathrm{O}_{2}$ (278.27): C, 64.74; $\mathrm{H}, 3.62 ; \mathrm{N}, 20.13 \%$. Found: $\mathrm{C}$, $64.54 ; \mathrm{H}, 3.56 ; \mathrm{N}, 20.38 \%$.

\subsection{General Procedure for the Synthesis of 3-(1H-1,2,3-Triazol-1-yl)Quinoline-2,4(1H,3H)-Diones 1a-d by} Employing $\mathrm{CuSO}_{4} / \mathrm{Cu}^{0} / \mathrm{DMF}$ Conditions (Table 2, Entries 1 and 3-5)

A solution of phenylacetylene (6a) $(1.287 \mathrm{~g}, 12.6 \mathrm{mmol})$ or propargyl alcohol $(6 \mathbf{b})$ (706 $\mathrm{mg}, 12.6 \mathrm{mmol})$ in DMF $(4 \mathrm{~mL})$ was added dropwise to a vigorously stirred mixture of 3-azidoquinoline-2,4(1H,3H)-dione 5 ( $12 \mathrm{mmol}), \mathrm{CuSO}_{4} \cdot 5 \mathrm{H}_{2} \mathrm{O}(300 \mathrm{mg}, 1.2 \mathrm{mmol})$, granular copper $(1.5 \mathrm{~g}, 24 \mathrm{mmol})$ and DMF $(24 \mathrm{~mL})$. The reaction mixture was stirred in darkness for $30 \mathrm{~min}$. Afterward, $\left(\mathrm{NH}_{4}\right)_{2} \mathrm{CO}_{3}(3.5 \mathrm{~g}, 36 \mathrm{mmol})$ and water $(12 \mathrm{~mL})$ were added to the resulting brown-black suspension and the stirring was continued for $10 \mathrm{~min}$. The reaction mixture was subjected to column chromatography with silica gel $(15 \mathrm{~g}$, column diameter of $1 \mathrm{~cm})$ as a stationary phase, and $10 \%$ ethanol in chloroform as a mobile phase. Combined fractions containing yellow eluate were washed with saturated aqueous $\mathrm{NH}_{4} \mathrm{Cl}(5 \times 50 \mathrm{~mL})$, dried $\left(\mathrm{Na}_{2} \mathrm{SO}_{4}\right)$, and concentrated under reduced pressure to afford pure products $\mathbf{1 a}-\mathbf{d}$, which were recrystallized from ethanol for analyses.

3-Methyl-3-(4-phenyl-1H-1,2,3-triazol-1-yl)quinoline-2,4(1H,3H)-dione (1a). Colorless solid, m.p. $217-219{ }^{\circ} \mathrm{C}$ (ethanol); $R_{\mathrm{f}}=0.35$ (30\% ethyl acetate in chloroform); ${ }^{1} \mathrm{H}$ NMR $\left(500 \mathrm{MHz}\right.$, DMSO- $\left.d_{6}\right) \delta$ $2.15\left(\mathrm{~s}, 3 \mathrm{H}, \mathrm{CH}_{3}\right), 7.22-7.27(\mathrm{~m}, 2 \mathrm{H}, \mathrm{H}-6, \mathrm{H}-8), 7.33-7.39\left(\mathrm{~m}, 1 \mathrm{H}, \mathrm{H}-4^{\mathrm{B}}\right), 7.45-7.50\left(\mathrm{~m}, 2 \mathrm{H}, \mathrm{H}-3^{\mathrm{B}}, \mathrm{H}-5^{\mathrm{B}}\right)$, 7.73-7.79 (m, 1H, H-7), 7.83-7.90 (m, 3H, H-5, H-2 $\left.{ }^{\mathrm{B}}, \mathrm{H}^{6} \mathrm{6}^{\mathrm{B}}\right), 8.89$ (s, 1H, H-5 $\left.{ }^{\mathrm{A}}\right), 11.48(\mathrm{~s}, 1 \mathrm{H}, \mathrm{H}-1){ }^{13} \mathrm{C}$ NMR $\left(126 \mathrm{MHz}\right.$, DMSO- $\left.d_{6}\right) \delta 23.1\left(\mathrm{CH}_{3}\right), 72.2(\mathrm{C}-3), 117.0(\mathrm{C}-8), 117.4(\mathrm{C}-4 \mathrm{a}), 122.4\left(\mathrm{C}-5^{\mathrm{A}}\right), 123.5(\mathrm{C}-6)$, $125.1\left(\mathrm{C}-2^{\mathrm{B}}, \mathrm{C}-6^{\mathrm{B}}\right), 127.7(\mathrm{C}-5), 128.0\left(\mathrm{C}-4^{\mathrm{B}}\right), 129.0\left(\mathrm{C}-3^{\mathrm{B}}, \mathrm{C}-5^{\mathrm{B}}\right), 130.6\left(\mathrm{C}-1^{\mathrm{B}}\right), 137.3(\mathrm{C}-7), 141.6(\mathrm{C}-8 \mathrm{a})$, $145.8\left(\mathrm{C}-4^{\mathrm{A}}\right), 168.5(\mathrm{C}-2), 190.7(\mathrm{C}-4)$; IR $\left(\mathrm{cm}^{-1}\right): v$ 3137, 2911, 1714, 1683, 1612, 1483, 1430, 1386, 1355, 1238, 1023, 808, 759, 690, 594; MS (EI) $m / z(\%): 319\left(2,[\mathrm{M}+1]^{+}\right), 318\left(8,[\mathrm{M}]^{+}\right), 117(14), 116(100)$, 102 (12), 89 (14); HRMS (ESI+): $m / z$ calcd for $\mathrm{C}_{18} \mathrm{H}_{15} \mathrm{~N}_{4} \mathrm{O}_{2}{ }^{+}[\mathrm{M}+\mathrm{H}]^{+} 319.1190$, found 319.1188. Anal. Calcd for $\mathrm{C}_{18} \mathrm{H}_{14} \mathrm{~N}_{4} \mathrm{O}_{2}$ (318.33): C, 67.91; H, 4.43; N, 17.60\%. Found: C, 67.80; H, 4.47; N, $17.89 \%$.

3-Phenyl-3-(4-phenyl-1H-1,2,3-triazol-1-yl)quinoline-2,4(1H,3H)-dione (1b). Colorless solid, m.p. 280-283 ${ }^{\circ} \mathrm{C}$ (ethanol); m.p. [9] $274-277{ }^{\circ} \mathrm{C}$ (ethanol); $R_{\mathrm{f}}=0.37$ (30\% ethyl acetate in chloroform); ${ }^{1} \mathrm{H}$ NMR $\left(500 \mathrm{MHz}\right.$, DMSO- $\left.d_{6}\right) \delta 7.12(\mathrm{~d}, 1 \mathrm{H}, J=8.1 \mathrm{~Hz}, \mathrm{H}-8), 7.16-7.20(\mathrm{~m}, 1 \mathrm{H}, \mathrm{H}-6), 7.31-7.37(\mathrm{~m}, 1 \mathrm{H}$, $\left.\mathrm{H}-4^{\mathrm{B}}\right), 7.40-7.47\left(\mathrm{~m}, 4 \mathrm{H}, \mathrm{H}-3^{\mathrm{B}}, \mathrm{H}-5^{\mathrm{B}}, \mathrm{H}-2^{\mathrm{C}}, \mathrm{H}^{-6} 6^{\mathrm{C}}\right), 7.49-7.55\left(\mathrm{~m}, 3 \mathrm{H}, \mathrm{H}-3^{\mathrm{C}}, \mathrm{H}-4^{\mathrm{C}}, \mathrm{H}-5^{\mathrm{C}}\right), 7.62-7.67(\mathrm{~m}$, $1 \mathrm{H}, \mathrm{H}-7), 7.80-7.84\left(\mathrm{~m}, 2 \mathrm{H}, \mathrm{H}-2^{\mathrm{B}}, \mathrm{H}-6^{\mathrm{B}}\right), 7.86(\mathrm{dd}, 1 \mathrm{H}, J=7.8,1.4 \mathrm{~Hz}, \mathrm{H}-5), 8.49\left(\mathrm{~s}, 1 \mathrm{H}, \mathrm{H}-5^{\mathrm{A}}\right), 11.68(\mathrm{~s}$, $1 \mathrm{H}, \mathrm{H}-1) ;{ }^{13} \mathrm{C}$ NMR $\left(126 \mathrm{MHz}, \mathrm{DMSO}-d_{6}\right) \delta 80.0(\mathrm{C}-3), 116.7$ (C-8), $119.2(\mathrm{C}-4 \mathrm{a}), 123.4\left(\mathrm{C}-5^{\mathrm{A}}\right), 123.5$ $(\mathrm{C}-6), 125.2\left(\mathrm{C}-2^{\mathrm{B}}, \mathrm{C}-6^{\mathrm{B}}\right), 127.6(\mathrm{C}-5), 128.0\left(\mathrm{C}-4^{\mathrm{B}}\right), 128.9\left(\mathrm{C}-2^{\mathrm{C}}, \mathrm{C}-6^{\mathrm{C}}\right), 129.0\left(\mathrm{C}-3^{\mathrm{B}}, \mathrm{C}-5^{\mathrm{B}}\right), 129.6\left(\mathrm{C}-3^{\mathrm{C}}\right.$, C-5 $), 129.9\left(\mathrm{C}-1^{\mathrm{C}}\right), 130.5\left(\mathrm{C}-1^{\mathrm{B}}\right), 130.6\left(\mathrm{C}-4^{\mathrm{C}}\right), 137.0(\mathrm{C}-7), 140.5(\mathrm{C}-8 \mathrm{a}), 145.3\left(\mathrm{C}-4^{\mathrm{A}}\right), 166.8(\mathrm{C}-2), 188.9$ (C-4); IR ( $\left.\mathrm{cm}^{-1}\right): v$ 3275, 3169, 1721, 1690, 1613, 1595, 1486, 1452, 1353, 854, 771, 756, 699, 666, 607; MS (EI) m/z (\%): $381\left(2,[\mathrm{M}+1]^{+}\right), 380\left(8,[\mathrm{M}]^{+}\right), 247$ (13), 237 (15), 236 (56), 220 (13), 218 (13), 120 (11), 117 (10), 116 (100), 102 (15), 92 (10), 89 (15), 77 (14); HRMS (ESI+): $m / z$ calcd for $\mathrm{C}_{23} \mathrm{H}_{17} \mathrm{~N}_{4} \mathrm{O}_{2}{ }^{+}[\mathrm{M}+\mathrm{H}]^{+}$ 381.1346, found 381.1341. Anal. Calcd for $\mathrm{C}_{23} \mathrm{H}_{16} \mathrm{~N}_{4} \mathrm{O}_{2}$ (380.40): $\mathrm{C}, 72.62 ; \mathrm{H}, 4.24 ; \mathrm{N}, 14.73 \%$. Found: $\mathrm{C}$, $72.40 ; \mathrm{H}, 4.23 ; \mathrm{N}, 14.90 \%$.

3-(4-(Hydroxymethyl)-1H-1,2,3-triazol-1-yl)-3-methylquinoline-2,4(1H,3H)-dione (1c). Colorless solid, m.p. $188-189{ }^{\circ} \mathrm{C}$ (ethanol); $R_{\mathrm{f}}=0.35$ (30\% ethyl acetate in chloroform); ${ }^{1} \mathrm{H}$ NMR $(500 \mathrm{MHz}$, DMSO- $\left.d_{6}\right) \delta 2.08\left(\mathrm{~s}, 3 \mathrm{H}, \mathrm{CH}_{3}\right), 4.55\left(\mathrm{~d}, 2 \mathrm{H}, J=5.6 \mathrm{~Hz}, \mathrm{CH}_{2}\right), 5.28(\mathrm{t}, 1 \mathrm{H}, J=5.6 \mathrm{~Hz}, \mathrm{OH}), 7.18-7.25(\mathrm{~m}$, 2H, H-6, H-8), 7.69-7.76 (m, 1H, H-7), 7.83 (dd, 1H, J = 8.1, 1.4 Hz, H-5), $8.26\left(\mathrm{~s}, 1 \mathrm{H}, \mathrm{H}-5^{\mathrm{A}}\right), 11.39(\mathrm{~s}, 1 \mathrm{H}$, 
$\mathrm{H}-1) ;{ }^{13} \mathrm{C}$ NMR $\left(126 \mathrm{MHz}, \mathrm{DMSO}-d_{6}\right) \delta 23.1\left(\mathrm{CH}_{3}\right), 55.0\left(\mathrm{CH}_{2}\right), 71.9(\mathrm{C}-3), 116.9(\mathrm{C}-8), 117.5(\mathrm{C}-4 \mathrm{a}), 123.3$ (C-6), $\left.123.7\left(\mathrm{C}-5^{\mathrm{A}}\right), 127.6(\mathrm{C}-5), 137.1(\mathrm{C}-7), 141.6(\mathrm{C}-8 \mathrm{a}), 147.4\left(\mathrm{C}-4^{\mathrm{A}}\right), 168.7(\mathrm{C}-2), 190.8(\mathrm{C}-4) ; \mathrm{IR}_{(\mathrm{cm}}^{-1}\right)$ : $v$ 3148, 2992, 2919, 1729, 1682, 1613, 1486, 1378, 1345, 1235, 1189, 1009, 751, 667, 590; MS (EI) m/z (\%): $273\left(2,[\mathrm{M}+1]^{+}\right), 272\left(13,[\mathrm{M}]^{+}\right), 185(68), 175(89), 174(45), 146(100), 128(58), 120(70), 119(75), 92(66)$, 91 (39), 77 (39), 65 (37), 55 (39), 42 (79); HRMS (ESI+): $m / z$ calcd for $\mathrm{C}_{13} \mathrm{H}_{13} \mathrm{~N}_{4} \mathrm{O}_{3}{ }^{+}[\mathrm{M}+\mathrm{H}]^{+} 273.0982$, found 273.0981. Anal. Calcd for $\mathrm{C}_{13} \mathrm{H}_{12} \mathrm{~N}_{4} \mathrm{O}_{3}$ (272.26): C, 57.35; H, 4.44; N, 20.58\%. Found: C, 57.20; $\mathrm{H}$, $4.42 ; \mathrm{N}, 20.83 \%$.

3-(4-(Hydroxymethyl)-1H-1,2,3-triazol-1-yl)-3-phenylquinoline-2,4(1H,3H)-dione dimethylformamide solvate (1d·DMF). Colorless solid, m.p. 139-143 ${ }^{\circ} \mathrm{C}$ (ethanol); m.p. [9] 116-135 ${ }^{\circ} \mathrm{C}$ (benzene); $R_{\mathrm{f}}=0.27$ (10\% ethanol in chloroform); ${ }^{1} \mathrm{H}$ NMR $\left(500 \mathrm{MHz}, \mathrm{DMSO}-\mathrm{d}_{6}\right) \delta 4.53\left(\mathrm{~d}, 2 \mathrm{H}, J=5.7 \mathrm{~Hz}, \mathrm{CH}_{2}\right), 5.22(\mathrm{t}, 1 \mathrm{H}$, $J=5.7 \mathrm{~Hz}, \mathrm{OH}), 7.09(\mathrm{~d}, 1 \mathrm{H}, J=8.1 \mathrm{~Hz}, \mathrm{H}-8), 7.13-7.18(\mathrm{~m}, 1 \mathrm{H}, \mathrm{H}-6), 7.36-7.42\left(\mathrm{~m}, 2 \mathrm{H}, \mathrm{H}-2^{\mathrm{C}}, \mathrm{H}_{-} 6^{\mathrm{C}}\right)$, 7.47-7.53 (m, 3H, H-3 $\left.{ }^{\mathrm{C}}, \mathrm{H}-4^{\mathrm{C}}, \mathrm{H}-5^{\mathrm{C}}\right), 7.59-7.65(\mathrm{~m}, 1 \mathrm{H}, \mathrm{H}-7), 7.77\left(\mathrm{~s}, 1 \mathrm{H}, \mathrm{H}-5^{\mathrm{A}}\right), 7.83(\mathrm{dd}, 1 \mathrm{H}, \mathrm{J}=7.8,1.3$ $\mathrm{Hz}, \mathrm{H}-5), 11.60$ (s, 1H, H-1); ${ }^{13} \mathrm{C}$ NMR (126 MHz, DMSO-d 6$) \delta 55.0\left(\mathrm{CH}_{2}\right), 79.7$ (C-3), $116.7(\mathrm{C}-8), 119.2$ (C-4a), $123.4(\mathrm{C}-6), 124.8\left(\mathrm{C}-5^{\mathrm{A}}\right), 127.5(\mathrm{C}-5), 128.8\left(\mathrm{C}-2^{\mathrm{C}}, \mathrm{C}-6^{\mathrm{C}}\right), 129.6\left(\mathrm{C}-3^{\mathrm{C}}, \mathrm{C}-5^{\mathrm{C}}\right), 130.2\left(\mathrm{C}-1^{\mathrm{C}}\right), 130.5$ $\left(\mathrm{C}-4^{\mathrm{C}}\right), 136.9(\mathrm{C}-7), 140.6(\mathrm{C}-8 \mathrm{a}), 146.8\left(\mathrm{C}-4^{\mathrm{A}}\right), 166.8(\mathrm{C}-2), 189.0(\mathrm{C}-4)$; IR $\left(\mathrm{cm}^{-1}\right): v 3392,3136,2926$, $1724,1692,1654,1613,1485,1438,1353,857,769,752,665,603 ; \mathrm{MS}$ (EI) $m / z(\%): 335\left(0.8,[\mathrm{M}+1]^{+}\right), 334$ $\left(4,[\mathrm{M}]^{+}\right), 305$ (37), 275 (18), 249 (30), 247 (27), 237 (50), 236 (100), 218 (35), 208 (18), 180 (20), 120 (33), 104 (23), 92 (23), 77 (34); HRMS (ESI+): $m / z$ calcd for $\mathrm{C}_{18} \mathrm{H}_{15} \mathrm{~N}_{4} \mathrm{O}_{3}{ }^{+}[\mathrm{M}+\mathrm{H}]^{+}$335.1139, found 335.1138. Anal. Calcd for $\mathrm{C}_{21} \mathrm{H}_{21} \mathrm{~N}_{5} \mathrm{O}_{4}$ (407.42): C, 61.91; H, 5.20; N, 17.19\%. Found: C, 61.89; H, 5.24; N, 17.28\%.

\subsection{Synthesis of Compound 1a by Employing $\mathrm{CuSO}_{4} \cdot 5 \mathrm{H}_{2} \mathrm{O} / \mathrm{L}-$ Ascorbic Acid/ $\mathrm{CH}_{2} \mathrm{Cl}_{2} /$ Water Conditions} (Table 2, Entry 2)

To a solution of azide $5 \mathbf{a}(162 \mathrm{mg}, 0.75 \mathrm{mmol})$ and phenylacetylene (6a) (153 mg, $1.5 \mathrm{mmol})$ in dichloromethane $(8 \mathrm{~mL})$ a solution of L-ascorbic acid $(106 \mathrm{mg}, 0.60 \mathrm{mmol})$ in water $(4 \mathrm{~mL})$, and a solution of $\mathrm{CuSO}_{4} \cdot 5 \mathrm{H}_{2} \mathrm{O}(15 \mathrm{mg}, 0.06 \mathrm{mmol})$ in water $(4 \mathrm{~mL})$ were added. The two-phase reaction mixture was stirred in darkness at room temperature for $48 \mathrm{~h}$. The reaction mixture was extracted with chloroform $(5 \times 30 \mathrm{~mL})$. The combined organic layers were dried $\left(\mathrm{Na}_{2} \mathrm{SO}_{4}\right)$, filtered, and evaporated to dryness. The residue was dissolved in chloroform (5 mL) and subjected to silica gel (30 g) column chromatography using 38\% ethyl acetate in hexane as eluent, affording compound 1a (199 mg, $63 \mathrm{mmol} \mathrm{83 \% ).}$

3.6. General Procedure for the Synthesis of (1-(2,4-Dioxo-1,2,3,4-Tetrahydroquinolin-3-yl)-1H-1,2,3-Triazol4-yl)Methyl Acetates 1e,f (Scheme 2)

Acetic anhydride $(12 \mathrm{~mL}, 12.9 \mathrm{~g}, 126 \mathrm{mmol})$ was added to a light yellow solution of compound 1c or $1 \mathbf{d}(6 \mathrm{mmol})$ in pyridine $(18 \mathrm{~mL})$ under stirring during $2 \mathrm{~min}$. The reaction mixture was stirred for $1 \mathrm{~h}$, followed by evaporation of volatiles under reduced pressure. The remaining pyridine was removed by co-distillation with ethanol $(6 \times 40 \mathrm{~mL})$. The residue was triturated with water $(300 \mathrm{~mL})$ to form a white precipitate which was collected by filtration on a sintered glass filter with suction, washed with water to neutral and dried to give acetates 1e or 1f. The crude product was recrystallized from the solvent indicated below.

(1-(3-Methyl-2,4-dioxo-1,2,3,4-tetrahydroquinolin-3-yl)-1H-1,2,3-triazol-4-yl)methyl acetate (1e). Compound 1e $(1.58 \mathrm{~g}, 5.04 \mathrm{mmol}, 84 \%)$ was prepared from 1c (1.63 g, $6.0 \mathrm{mmol})$. Pale yellow solid, m.p. $145-148{ }^{\circ} \mathrm{C}$ (ethyl acetate); $R_{\mathrm{f}}=0.33$ (5\% ethanol in chloroform); ${ }^{1} \mathrm{H}$ NMR $\left(500 \mathrm{MHz}, \mathrm{DMSO}-d_{6}\right) \delta$ $2.06\left(\mathrm{~s}, 3 \mathrm{H}, \mathrm{COCH}_{3}\right), 2.09\left(\mathrm{~s}, 3 \mathrm{H}, \mathrm{C}-3-\mathrm{CH}_{3}\right), 5.16\left(\mathrm{~s}, 2 \mathrm{H}, \mathrm{CH}_{2}\right), 7.19-7.26(\mathrm{~m}, 2 \mathrm{H}, \mathrm{H}-6, \mathrm{H}-8), 7.70-7.77(\mathrm{~m}$, 1H, H-7), $7.83(\mathrm{dd}, 1 \mathrm{H}, J=8.0,1.4 \mathrm{~Hz}, \mathrm{H}-5), 8.45\left(\mathrm{~s}, 1 \mathrm{H}, \mathrm{H}-5^{\mathrm{A}}\right), 11.40(\mathrm{~s}, 1 \mathrm{H}, \mathrm{H}-1)$; ${ }^{13} \mathrm{C} \mathrm{NMR}(126 \mathrm{MHz}$, DMSO-d $\left.d_{6}\right) \delta 20.6\left(\mathrm{COCH}_{3}\right), 23.2\left(\mathrm{C}-3-\mathrm{CH}_{3}\right), 57.1\left(\mathrm{CH}_{2}\right), 72.4(\mathrm{C}-3), 116.9(\mathrm{C}-8), 117.5(\mathrm{C}-4 \mathrm{a}), 123.3(\mathrm{C}-6)$, $125.8\left(\mathrm{C}-5^{\mathrm{A}}\right), 127.6(\mathrm{C}-5), 137.1(\mathrm{C}-7), 141.4\left(\mathrm{C}-4^{\mathrm{A}}\right), 141.6(\mathrm{C}-8 \mathrm{a}), 168.6(\mathrm{C}-2), 170.1\left(\mathrm{COCH}_{3}\right), 190.7$

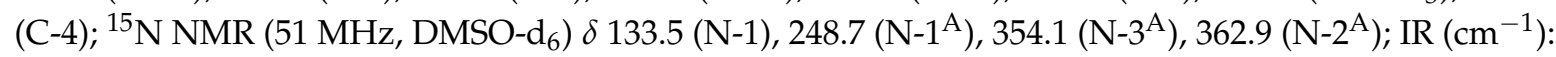
$v$ 3467, 3249, 3148, 2920, 1722, 1685, 1613, 1485, 1439, 1384, 1355, 1239, 1028, 759, 666; MS (EI) m/z (\%): 
$315\left(2,[\mathrm{M}+1]^{+}\right), 314\left(11,[\mathrm{M}]^{+}\right), 244(22), 201(22), 175(71), 174(31), 146(43), 128$ (26), $120(25), 119(27)$, 92 (24), 55 (20), 43 (100), 42 (26); HRMS (ESI+): $m / z$ calcd for $\mathrm{C}_{15} \mathrm{H}_{15} \mathrm{~N}_{4} \mathrm{O}_{4}{ }^{+}[\mathrm{M}+\mathrm{H}]^{+} 315.1088$, found 315.1087. Anal. Calcd for $\mathrm{C}_{15} \mathrm{H}_{14} \mathrm{~N}_{4} \mathrm{O}_{4}$ (314.30): C, 57.32; $\mathrm{H}, 4.49 ; \mathrm{N}, 17.83 \%$. Found: C, 57.32; $\mathrm{H}, 4.59$; $\mathrm{N}, 17.58 \%$.

(1-(2,4-Dioxo-3-phenyl-1,2,3,4-tetrahydroquinolin-3-yl)-1H-1,2,3-triazol-4-yl)methyl acetate (1f). Compound $1 \mathrm{f}(1.92 \mathrm{~g}, 5.1 \mathrm{mmol}, 85 \%)$ was prepared from $1 \mathrm{~d}(2.01 \mathrm{~g}, 6.0 \mathrm{mmol})$. Colorless crystals, m.p. $130-134{ }^{\circ} \mathrm{C}$ (ethanol, $80 \%$ yield of recrystallization); $R_{\mathrm{f}}=0.40$ (5\% ethanol in chloroform); ${ }^{1} \mathrm{H}$ NMR $\left(500 \mathrm{MHz}, \mathrm{DMSO}-d_{6}\right) \delta 2.04\left(\mathrm{~s}, 3 \mathrm{H}, \mathrm{CH}_{3}\right), 5.13\left(\mathrm{~s}, 2 \mathrm{H}, \mathrm{CH}_{2}\right), 7.09(\mathrm{~d}, 1 \mathrm{H}, J=8.1 \mathrm{~Hz}, \mathrm{H}-8), 7.13-7.18(\mathrm{~m}$,

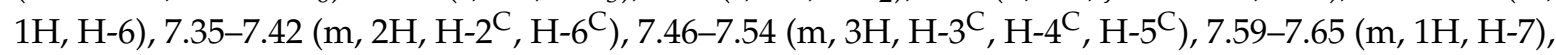
$7.83(\mathrm{dd}, 1 \mathrm{H}, J=7.8,1.3 \mathrm{~Hz}), 8.07\left(\mathrm{~s}, 1 \mathrm{H}, \mathrm{H}-5^{\mathrm{A}}\right), 11.62(\mathrm{~s}, 1 \mathrm{H}, \mathrm{H}-1) ;{ }^{13} \mathrm{C}$ NMR $\left(126 \mathrm{MHz}, \mathrm{DMSO}-d_{6}\right) \delta$ $20.7\left(\mathrm{CH}_{3}\right), 57.1\left(\mathrm{CH}_{2}\right), 79.9(\mathrm{C}-3), 116.7(\mathrm{C}-8), 119.3(\mathrm{C}-4 \mathrm{a}), 123.4(\mathrm{C}-6), 127.0\left(\mathrm{C}-5^{\mathrm{A}}\right), 127.5(\mathrm{C}-5), 128.8$ $\left(\mathrm{C}-2^{\mathrm{C}}, \mathrm{C}-6^{\mathrm{C}}\right), 129.6\left(\mathrm{C}-3^{\mathrm{C}}, \mathrm{C}-5^{\mathrm{C}}\right), 130.0\left(\mathrm{C}-1^{\mathrm{C}}\right), 130.6\left(\mathrm{C}-4^{\mathrm{C}}\right), 136.9(\mathrm{C}-7), 140.5(\mathrm{C}-8 \mathrm{a}), 140.8\left(\mathrm{C}-4^{\mathrm{A}}\right), 166.8$ (C-2), $170.1\left(\mathrm{COCH}_{3}\right), 188.8$ (C-4); IR (cm-1): $v$ 3501, 3155, 2920, 1722, 1707, 1686, 1614, 1594, 1484, 1358, 1252, 1229, 1063, 857, 759; MS (EI) m/z (\%): 377 (1, [M + 1]+), 376 (6, [M]+), 306 (16), 289 (18), 288 (54), 263 (15), 237 (50), 236 (100), 218 (34), 180 (14), 141 (14), 120 (24), 92 (14), 77 (19), 43 (16); HRMS (ESI+): $m / z$ calcd for $\mathrm{C}_{20} \mathrm{H}_{17} \mathrm{~N}_{4} \mathrm{O}_{4}{ }^{+}[\mathrm{M}+\mathrm{H}]^{+} 377.1244$, found 377.1241.

3.7. General Procedure for the Synthesis of 3-(1H-1,2,3-Triazol-1-yl)-1-(prop-2-yn-1-yl)Quinoline-2,4(1H,3H)Diones 7 (Table 3)

The mixture of the appropriate compound $\mathbf{1 a}, \mathbf{b}, \mathbf{e}, \mathbf{f}(8.00 \mathrm{mmol})$, potassium carbonate $(3.32 \mathrm{~g}$, $24 \mathrm{mmol})$, and DMF (40 mL) was stirred for $40 \mathrm{~min}$. During this time, the original yellow color of the suspension changed to orange. Afterwards, under continued stirring, an $80 \%$ solution of propargyl bromide (6c) in toluene $(1.78 \mathrm{~g}, 12 \mathrm{mmol})$ diluted with DMF $(20 \mathrm{~mL})$ was added dropwise during $1 \mathrm{~min}$ and stirring was continued for $90 \mathrm{~min}$. Then the mixture was reduced in vacuo and then toluene $(50 \mathrm{~mL})$ was added and the whole evaporated in vacuo at $50{ }^{\circ} \mathrm{C}$. This was repeated seven times in order to remove traces of DMF. The residual light brown solid was suspended in chloroform $(100 \mathrm{~mL})$ and the suspension was acidified with $0.5 \mathrm{M} \mathrm{HCl}$, whereas carbon dioxide was evolved owing to decomposition of unreacted potassium carbonate. The formed emulsion was diluted with water, organic phase was separated and aqueous phase was extracted with chloroform $(5 \times 40 \mathrm{~mL})$. The organic phases were combined, dried $\left(\mathrm{Na}_{2} \mathrm{SO}_{4}\right)$, filtered and taken down in vacuo. The residual solid TLC pure product was crystallized from a suitable solvent. The yields of prepared compounds 7 are given in Table 3.

3-Methyl-3-(4-phenyl-1H-1,2,3-triazol-1-yl)-1-(prop-2-yn-1-yl)quinoline-2,4(1H,3H)-dione (7a). Colorless crystals, m.p. $187-189{ }^{\circ} \mathrm{C}$ (benzene); $R_{\mathrm{f}}=0.63$ (30\% ethyl acetate in chloroform); ${ }^{1} \mathrm{H}$ NMR $\left(500 \mathrm{MHz}, \mathrm{DMSO}-d_{6}\right) \delta 2.16\left(\mathrm{~s}, 3 \mathrm{H}, \mathrm{CH}_{3}\right), 3.39(\mathrm{dd}, 1 \mathrm{H}, J=2.3,2.3 \mathrm{~Hz}, \mathrm{C} \equiv \mathrm{CH}), 4.90(\mathrm{dd}, 1 \mathrm{H}, J=18.1$, $2.3 \mathrm{~Hz}, \mathrm{~N}-1-\mathrm{CH} \alpha), 4.97(\mathrm{dd}, 1 \mathrm{H}, \mathrm{J}=18.1,2.3 \mathrm{~Hz}, \mathrm{~N}-1-\mathrm{CH} \beta), 7.34-7.42\left(\mathrm{~m}, 2 \mathrm{H}, \mathrm{H}-6, \mathrm{H}-4^{\mathrm{B}}\right), 7.48(\mathrm{dd}, 2 \mathrm{H}$, $\left.J=7.7,7.7 \mathrm{~Hz}, \mathrm{H}-3^{\mathrm{B}}, \mathrm{H}-5^{\mathrm{B}}\right), 7.61(\mathrm{~d}, 1 \mathrm{H}, J=8.4 \mathrm{~Hz}, \mathrm{H}-8), 7.84-7.89\left(\mathrm{~m}, 2 \mathrm{H}, \mathrm{H}-2^{\mathrm{B}},{\mathrm{H}-6^{\mathrm{B}}}^{\mathrm{B}}\right), 7.89-7.95(\mathrm{~m}$, $1 \mathrm{H}, \mathrm{H}-7), 8.00(\mathrm{dd}, J=7.7,1.5 \mathrm{~Hz}, \mathrm{H}-5), 8.89\left(\mathrm{~s}, 1 \mathrm{H}, \mathrm{H}-5^{\mathrm{A}}\right) ;{ }^{13} \mathrm{C}$ NMR $\left(126 \mathrm{MHz}, \mathrm{DMSO}-d_{6}\right) \delta 23.3\left(\mathrm{CH}_{3}\right)$, $32.7\left(\mathrm{~N}-1-\mathrm{CH}_{2}\right), 72.6(\mathrm{C}-3), 75.4(\mathrm{C} \equiv \mathrm{CH}), 78.2(\mathrm{C} \equiv \mathrm{CH}), 116.7(\mathrm{C}-8), 119.0(\mathrm{C}-4 \mathrm{a}), 122.5\left(\mathrm{C}-5^{\mathrm{A}}\right), 124.2$ (C-6), $125.1\left(\mathrm{C}-2^{\mathrm{B}}, \mathrm{C}-6^{\mathrm{B}}\right), 128.1\left(\mathrm{C}-4^{\mathrm{B}}\right), 128.2(\mathrm{C}-5), 129.0\left(\mathrm{C}-3^{\mathrm{B}}, \mathrm{C}-5^{\mathrm{B}}\right), 130.5\left(\mathrm{C}-1^{\mathrm{B}}\right), 137.3(\mathrm{C}-7), 140.8$ (C-8a), $145.9\left(\mathrm{C}-4^{\mathrm{A}}\right), 167.7$ (C-2), 189.7 (C-4); IR ( $\left.\mathrm{cm}^{-1}\right): v$ 3261, 3173, 2122, 1713, 1678, 1601, 1469, 1427, 1381, 1368, 1353, 1306, 1189, 769, 754; MS (EI) $m / z(\%): 357\left(2,[\mathrm{M}+1]^{+}\right), 356\left(8,[\mathrm{M}]^{+}\right), 259(10), 128(11)$, 117 (16), 116 (100), 102 (17), 90 (11), 89 (16), 77 (10), 76 (10); HRMS (ESI+): $m / z$ calcd for $\mathrm{C}_{21} \mathrm{H}_{17} \mathrm{~N}_{4} \mathrm{O}_{2}{ }^{+}$ $[\mathrm{M}+\mathrm{H}]^{+}$357.1346, found 357.1342. Anal. Calcd for $\mathrm{C}_{21} \mathrm{H}_{16} \mathrm{~N}_{4} \mathrm{O}_{2}$ (356.38): C, 70.77; $\mathrm{H}, 4.53 ; \mathrm{N}, 15.72 \%$. Found: C, $70.81 ; \mathrm{H}, 4.58 ; \mathrm{N}, 15.82 \%$.

3-Phenyl-3-(4-phenyl-1H-1,2,3-triazol-1-yl)-1-(prop-2-yn-1-yl)quinoline-2,4(1H,3H)-dione (7b). Colorless crystals, m.p. $232-234{ }^{\circ} \mathrm{C}$ (ethanol); $R_{\mathrm{f}}=0.69$ (30\% ethyl acetate in chloroform); ${ }^{1} \mathrm{H}$ NMR $\left(500 \mathrm{MHz}, \mathrm{CDCl}_{3}\right) \delta 2.34(\mathrm{dd}, 1 \mathrm{H}, J=2.4,2.4 \mathrm{~Hz}), 4.51(\mathrm{dd}, 1 \mathrm{H}, J=17.8,2.3 \mathrm{~Hz}), 5.37(\mathrm{dd}, 1 \mathrm{H}, J=17.8$, $2.3 \mathrm{~Hz}), 7.23(\mathrm{dd}, 1 \mathrm{H}, J=7.6,7.6 \mathrm{~Hz}), 7.26(\mathrm{~s}, 1 \mathrm{H}), 7.27-7.31(\mathrm{~m}, 1 \mathrm{H}), 7.32-7.40(\mathrm{~m}, 3 \mathrm{H}), 7.43-7.51(\mathrm{~m}$, $3 \mathrm{H}), 7.51-7.56(\mathrm{~m}, 2 \mathrm{H}), 7.62-7.69(\mathrm{~m}, 1 \mathrm{H}), 7.73-7.81(\mathrm{~m}, 2 \mathrm{H}), 8.05(\mathrm{dd}, 1 \mathrm{H}, J=7.7,1.4 \mathrm{~Hz}) ;{ }^{13} \mathrm{C}$ NMR 
$\left(126 \mathrm{MHz} \mathrm{CDCl}_{3}\right) \delta 33.6,73.6,76.9,79.6,115.8,121.0,122.3,124.6,126.0,128.1,128.8,129.0,129.2,130.0$, 130.1, 130.7, 131.3, 136.9, 140.6, 146.0, 165.8, 187.5; IR $\left(\mathrm{cm}^{-1}\right): v$ 3197, 2983, 2118, 1716, 1680, 1603, 1468, 1448, 1304, 1039, 870, 760, 752, 694; MS (EI) m/z (\%): $419\left(13,[\mathrm{M}+1]^{+}\right), 418\left(75,[\mathrm{M}]^{+}\right), 390(43)$, 287 (22), 286 (31), 285 (89), 276 (25), 275 (100), 274 (28), 259 (70), 248 (46), 235 (53), 145 (52), 116 (95), 44 (99); HRMS (ESI+): $m / z$ calcd for $\mathrm{C}_{26} \mathrm{H}_{19} \mathrm{~N}_{4} \mathrm{O}_{2}{ }^{+}[\mathrm{M}+\mathrm{H}]^{+}$419.1503, found 419.1502. Anal. Calcd for $\mathrm{C}_{26} \mathrm{H}_{18} \mathrm{~N}_{4} \mathrm{O}_{2}$ : C, 74.63; H, 4.34; N, 13.39\%. Found: C, 74.45; H, 4.40; N, 13.43\%.

(1-(3-Methyl-2,4-dioxo-1-(prop-2-yn-1-yl)-1,2,3,4-tetrahydroquinolin-3-yl)-1H-1,2,3-triazol-4-yl)methyl acetate (7c). Colorless crystals, m.p. $159-161{ }^{\circ} \mathrm{C}$ (ethyl acetate); $R_{\mathrm{f}}=0.29$ (30\% ethyl acetate in chloroform); ${ }^{1} \mathrm{H}$ NMR (500 MHz, DMSO-d 6 ) $\delta 2.06$ (s, 3H, COCH 3 ), $2.10\left(\mathrm{~s}, 3 \mathrm{H}, \mathrm{C}-3-\mathrm{CH}_{3}\right), 3.37$ (dd, $1 \mathrm{H}, J=2.4,2.4 \mathrm{~Hz}, \mathrm{C} \equiv \mathrm{CH}), 4.84(\mathrm{dd}, 1 \mathrm{H}, J=18.1,2.4 \mathrm{~Hz}, \mathrm{~N}-1-\mathrm{CH} \alpha), 4.95(\mathrm{dd}, 1 \mathrm{H}, J=18.1,2.4 \mathrm{~Hz}$, $\mathrm{N}-1-\mathrm{CH} \beta), 5.17$ (s, 2H, OCH 2$), 7.37(\mathrm{dd}, 1 \mathrm{H}, J=7.5,7.5 \mathrm{~Hz}, \mathrm{H}-6), 7.58$ (d, 1H, J = 8.4 Hz, H-8), 7.87-7.93 $(\mathrm{m}, 1 \mathrm{H}, \mathrm{H}-7), 7.96(\mathrm{dd}, 1 \mathrm{H}, J=7.7,1.5 \mathrm{~Hz}, \mathrm{H}-5), 8.46\left(\mathrm{~s}, 1 \mathrm{H}, \mathrm{H}-5^{\mathrm{A}}\right) ;{ }^{13} \mathrm{C} \mathrm{NMR}\left(126 \mathrm{MHz}, \mathrm{DMSO}-d_{6}\right) \delta$ $20.6\left(\mathrm{COCH}_{3}\right), 23.4\left(\mathrm{C}-3-\mathrm{CH}_{3}\right), 32.6\left(\mathrm{~N}-1-\mathrm{CH}_{2}\right), 57.1\left(\mathrm{OCH}_{2}\right), 72.8(\mathrm{C}-3), 75.3(\mathrm{C} \equiv \mathrm{CH}), 78.2(\mathrm{C} \equiv \mathrm{CH})$, 116.6 (C-8), 119.2 (C-4a), 124.0 (C-6), $126.0\left(\mathrm{C}-5^{\mathrm{A}}\right), 128.0(\mathrm{C}-5), 137.1(\mathrm{C}-7), 140.7(\mathrm{C}-8 \mathrm{a}), 141.5\left(\mathrm{C}-4^{\mathrm{A}}\right)$, $167.8(\mathrm{C}-2), 170.1\left(\mathrm{COCH}_{3}\right), 189.63(\mathrm{C}-4) ;{ }^{15} \mathrm{~N}$ NMR $\left(51 \mathrm{MHz}, \mathrm{DMSO}-d_{6}\right) \delta 134.4(\mathrm{~N} 1), 247.9\left(\mathrm{~N}-1^{\mathrm{A}}\right)$, $354.0\left(\mathrm{~N}-3^{\mathrm{A}}\right), 363.4\left(\mathrm{~N}-2^{\mathrm{A}}\right)$; IR $\left(\mathrm{cm}^{-1}\right): v$ 3256, 3152, 2122, 1721, 1687, 1604, 1471, 1383, 1306, 1246, 1194, 1053, 1008, 756; MS (EI) $m / z(\%): 353\left(3,[\mathrm{M}+1]^{+}\right), 352\left(12,[\mathrm{M}]^{+}\right), 213(69), 212(34), 184(19), 156(32)$, 146 (17), 130 (19), 129 (21), 128 (22), 77 (17), 57 (16), 55 (23), 43 (100), 42 (17); HRMS (ESI+): $\mathrm{m} / z$ calcd for $\mathrm{C}_{18} \mathrm{H}_{17} \mathrm{~N}_{4} \mathrm{O}_{4}{ }^{+}\left([\mathrm{M}+\mathrm{H}]^{+}\right)$: 353.1244, found 353.1246. Anal. Calcd for $\mathrm{C}_{18} \mathrm{H}_{16} \mathrm{~N}_{4} \mathrm{O}_{4}$ (352.34): C, 61.36; H, 4.58; N, 15.90\%. Found: C, 61.27; H, 4.64; N, 15.87\%.

(1-(2,4-Dioxo-3-phenyl-1-(prop-2-yn-1-yl)-1,2,3,4-tetrahydroquinolin-3-yl)-1H-1,2,3-triazol-4-yl)methyl acetate (7d). Colorless crystals, m.p. $210-214{ }^{\circ} \mathrm{C} ; R_{\mathrm{f}}=0.66$ (5\% ethanol in chloroform); ${ }^{1} \mathrm{H}$ NMR $\left(500 \mathrm{MHz}, \mathrm{DMSO}-d_{6}\right) \delta 2.05\left(\mathrm{~s}, 3 \mathrm{H}, \mathrm{COCH}_{3}\right), 3.41(\mathrm{dd}, 1 \mathrm{H}, J=2.4,2.3 \mathrm{~Hz}, \mathrm{C} \equiv \mathrm{CH}), 4.80(\mathrm{dd}, 1 \mathrm{H}, J=18.0$, $2.3 \mathrm{~Hz}, \mathrm{~N}-1-\mathrm{CH} \alpha), 5.09-5.20\left(\mathrm{~m}, 3 \mathrm{H}, \mathrm{N}-1-\mathrm{CH} \beta, \mathrm{OCH}_{2}\right), 7.24-7.32$ (m, 3H, H-6, H-2 $\left.{ }^{\mathrm{C}}, \mathrm{H}^{-6}{ }^{\mathrm{C}}\right), 7.41-7.51$ $\left(\mathrm{m}, 4 \mathrm{H}, \mathrm{H}-8, \mathrm{H}-3^{\mathrm{C}}, \mathrm{H}-4^{\mathrm{C}}, \mathrm{H}-5^{\mathrm{C}}\right), 7.73-7.79$ (m, 1H, H-7), $7.92(\mathrm{dd}, 1 \mathrm{H}, J=7.7,1.5 \mathrm{~Hz}, \mathrm{H}-5), 8.15$ (s, 1H, H-5 $\left.{ }^{\mathrm{A}}\right) ;{ }^{13} \mathrm{C}$ NMR $\left(126 \mathrm{MHz}, \mathrm{DMSO}-d_{6}\right) \delta 20.6\left(\mathrm{COCH}_{3}\right), 33.1\left(\mathrm{~N}-1-\mathrm{CH}_{2}\right), 57.1\left(\mathrm{OCH}_{2}\right), 75.5(\mathrm{C} \equiv \mathrm{CH})$, $77.9(\mathrm{C} \equiv \mathrm{CH}), 80.0(\mathrm{C}-3), 116.3(\mathrm{C}-8), 120.9(\mathrm{C}-4 \mathrm{a}), 124.2(\mathrm{C}-6), 127.1\left(\mathrm{C}-5^{\mathrm{A}}\right), 127.8(\mathrm{C}-5), 128.6\left(\mathrm{C}-2^{\mathrm{C}}\right.$, C-6 $\left.{ }^{\mathrm{C}}\right), 129.5\left(\mathrm{C}-3^{\mathrm{C}}, \mathrm{C}-5^{\mathrm{C}}\right), 129.9\left(\mathrm{C}-1^{\mathrm{C}}\right), 130.7\left(\mathrm{C}-4^{\mathrm{C}}\right), 136.7(\mathrm{C}-7), 140.0(\mathrm{C}-8 \mathrm{a}), 140.9\left(\mathrm{C}-4^{\mathrm{A}}\right), 165.8(\mathrm{C}-2)$, $170.1\left(\mathrm{COCH}_{3}\right), 187.7(\mathrm{C}-4)$; IR (cm $\left.{ }^{-1}\right): v$ 3227, 3152, 2116, 1736, 1715, 1683, 1602, 1467, 1379, 1303, 1251, 1036, 764, 747, 694; MS (EI) m/z (\%): $415\left(2,[\mathrm{M}+1]^{+}\right), 414\left(7,[\mathrm{M}]^{+}\right), 313(26), 275(72), 274(63), 246(28)$, 235 (31), 218 (29), 217 (30), 156 (26), 130 (29), 105 (22), 104 (29), 103 (22), 43 (100); HRMS (ESI+): m/z calcd for $\mathrm{C}_{23} \mathrm{H}_{19} \mathrm{~N}_{4} \mathrm{O}_{4}{ }^{+}[\mathrm{M}+\mathrm{H}]^{+}$415.1401, found 415.1403. Anal. Calcd for $\mathrm{C}_{23} \mathrm{H}_{18} \mathrm{~N}_{4} \mathrm{O}_{4}$ : C, 66.66; $\mathrm{H}$, 4.38 ; N, 13.52\%. Found: C, 66.45; H, 4.39; N, 13.35\%.

\subsection{General Procedure for the Synthesis of Bis-Triazoles $2 a, b, d, e, g, h, j, k$ by Employing $\mathrm{CuSO}_{4} / \mathrm{Cu}^{0} / \mathrm{DMF}$ Conditions (Table 4, Entries 1, 2, 4, 8, 10,11, 13 and 14)}

A solution of azidobenzene $(\mathbf{8 b}, 197 \mathrm{mg}, 1.65 \mathrm{mmol})$ or (azidomethyl)benzene (8a, $220 \mathrm{mg}$, $1.65 \mathrm{mmol})$ in DMF $(4 \mathrm{~mL})$ was added to a vigorously stirred mixture of the appropriate $\mathrm{N}$-propargylquinoline-2,4(1H,3H)-dione $7(1.5 \mathrm{mmol}), \mathrm{CuSO}_{4} \cdot 5 \mathrm{H}_{2} \mathrm{O}(38 \mathrm{mg}, 0.15 \mathrm{mmol})$ and granular copper $(191 \mathrm{mg}, 3.05 \mathrm{mmol})$ in DMF $(5 \mathrm{~mL})$. The reaction mixture was stirred in darkness at room temperature for the time given in Table 4 . The color of the mixture became brown-black. Then, $\left(\mathrm{NH}_{4}\right)_{2} \mathrm{CO}_{3}(432 \mathrm{mg}, 4.5 \mathrm{mmol})$ and water $(2 \mathrm{~mL})$ were added to the reaction mixture and the stirring was continued for $10 \mathrm{~min}$. The reaction mixture was poured into a narrow $(1 \mathrm{~cm}$ in diameter) column of silica gel $(15 \mathrm{~g})$. The organic portion was eluted with 10\% ethanol in chloroform (approximately $150 \mathrm{~mL})$. The yellow eluate was washed with saturated aqueous $\mathrm{NH}_{4} \mathrm{Cl}(50 \mathrm{~mL})$, dried over anhydrous sodium sulfate, filtered, and the solvent was removed by rotary evaporation in vacuo. The TLC pure product thus prepared, with the exception of compounds $\mathbf{2} \mathbf{d}, \mathbf{e}, \mathbf{k}$, was crystallized from suitable solvent. The yields of prepared compounds 2 are given in Table 4. 
1-((1-Benzyl-1H-1,2,3-triazol-4-yl)methyl)-3-methyl-3-(4-phenyl-1H-1,2,3-triazol-1-yl)quinoline-2,4 (1H,3H)-dione (2a). Colorless crystals, m.p. 202-204 ${ }^{\circ} \mathrm{C}$ (ethanol); $R_{\mathrm{f}}=0.40$ (30\% ethyl acetate in chloroform); ${ }^{1} \mathrm{H}$ NMR (500 MHz, DMSO-d 6 ) $\delta 2.18\left(\mathrm{~s}, 3 \mathrm{H}, \mathrm{CH}_{3}\right), 5.24(\mathrm{~d}, 1 \mathrm{H}, J=16.2 \mathrm{~Hz}, \mathrm{~N}-1-\mathrm{CH} \alpha)$, $5.49(\mathrm{~d}, 1 \mathrm{H}, J=16.2 \mathrm{~Hz}, \mathrm{~N}-1-\mathrm{CH} \beta), 5.58\left(\mathrm{~s}, 2 \mathrm{H}, \mathrm{N}-1^{\mathrm{D}}-\mathrm{CH}_{2}\right), 7.24-7.29\left(\mathrm{~m}, 2 \mathrm{H}, \mathrm{H}-2^{\mathrm{E}}, \mathrm{H}^{-6}{ }^{\mathrm{E}}\right), 7.29-7.40$ $\left(\mathrm{m}, 5 \mathrm{H}, \mathrm{H}-6, \mathrm{H}-4^{\mathrm{B}}, \mathrm{H}-3^{\mathrm{E}}, \mathrm{H}-4^{\mathrm{E}}, \mathrm{H}-5^{\mathrm{E}}\right), 7.49\left(\mathrm{dd}, 2 \mathrm{H}, \mathrm{J}=7.7,7.7 \mathrm{~Hz}, \mathrm{H}-3^{\mathrm{B}}, \mathrm{H}-5^{\mathrm{B}}\right), 7.67(\mathrm{~d}, 1 \mathrm{H}, J=8.5 \mathrm{~Hz}$, H-8), 7.79-7.90 (m, 3H, H-7, H-2 $\left.{ }^{\mathrm{B}}, \mathrm{H}_{-}{ }^{\mathrm{B}}\right), 7.96$ (dd, 1H, J = 7.7, $\left.1.4 \mathrm{~Hz}, \mathrm{H}-5\right), 8.16\left(\mathrm{~s}, 1 \mathrm{H}, \mathrm{H}^{-} 5^{\mathrm{D}}\right), 8.87$ (s, $\left.1 \mathrm{H}, \mathrm{H}-5^{\mathrm{A}}\right) ;{ }^{13} \mathrm{C}$ NMR $\left(126 \mathrm{MHz}, \mathrm{DMSO}-d_{6}\right) \delta 23.4\left(\mathrm{CH}_{3}\right), 38.7\left(\mathrm{~N}-1-\mathrm{CH}_{2}\right), 52.8\left(\mathrm{~N}-1^{\mathrm{D}}-\mathrm{CH}_{2}\right), 72.8(\mathrm{C}-3)$, $116.7(\mathrm{C}-8), 119.1(\mathrm{C}-4 \mathrm{a}), 122.5\left(\mathrm{C}-5^{\mathrm{A}}\right), 123.8\left(\mathrm{C}-5^{\mathrm{D}}\right), 123.9(\mathrm{C}-6), 125.1\left(\mathrm{C}-2^{\mathrm{B}}, \mathrm{C}-6^{\mathrm{B}}\right), 127.9\left(\mathrm{C}-2^{\mathrm{E}}, \mathrm{C}-6^{\mathrm{E}}\right)$, $128.0\left(\mathrm{C}-4^{\mathrm{B}}\right), 128.1(\mathrm{C}-5), 128.1\left(\mathrm{C}-4^{\mathrm{E}}\right), 128.7\left(\mathrm{C}-3^{\mathrm{E}}, \mathrm{C}-5^{\mathrm{E}}\right), 129.1\left(\mathrm{C}-3^{\mathrm{B}}, \mathrm{C}-5^{\mathrm{B}}\right), 130.6\left(\mathrm{C}-1^{\mathrm{B}}\right), 136.0\left(\mathrm{C}-1^{\mathrm{E}}\right)$,

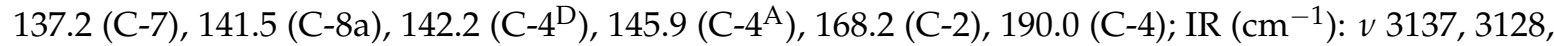
1711, 1673, 1600, 1471, 1387, 1051, 768, 761, 718, 694; MS (EI) $m / z(\%): 490\left(2,[\mathrm{M}+1]^{+}\right), 489\left(6,[\mathrm{M}]^{+}\right)$, 289 (13), 145 (17), 144 (16), 117 (11), 116 (44), 91 (100), 90 (10), 89 (12); HRMS (ESI+): $m / z$ calcd for $\mathrm{C}_{28} \mathrm{H}_{24} \mathrm{~N}_{7} \mathrm{O}_{2}{ }^{+}[\mathrm{M}+\mathrm{H}]^{+}$490.1986, found 490.1981. Anal. Calcd for $\mathrm{C}_{28} \mathrm{H}_{23} \mathrm{~N}_{7} \mathrm{O}_{2}(489.53) \mathrm{C}, 68.70 ; \mathrm{H}$, 4.74; N, 20.03. Found: C, 68.71; H, 4.78; N, 20.36.

3-Methyl-3-(4-phenyl-1H-1,2,3-triazol-1-yl)-1-((1-phenyl-1H-1,2,3-triazol-4-yl)methyl)quinoline-2,4 (1H,3H)-dione (2b). Colorless crystals, m.p. $194-197{ }^{\circ} \mathrm{C}$ (benzene); $R_{\mathrm{f}}=0.48$ (30\% ethyl acetate in chloroform); ${ }^{1} \mathrm{H}$ NMR (500 MHz, DMSO-d 6 ) $\delta 2.23\left(\mathrm{~s}, 3 \mathrm{H}, \mathrm{CH}_{3}\right), 5.33(\mathrm{~d}, 1 \mathrm{H}, \mathrm{J}=16.4 \mathrm{~Hz}, \mathrm{~N}-1-\mathrm{CH} \alpha)$, $5.62(\mathrm{~d}, 1 \mathrm{H}, \mathrm{J}=16.4 \mathrm{~Hz}, \mathrm{~N}-1-\mathrm{CH} \beta), 7.31-7.39\left(\mathrm{~m}, 2 \mathrm{H}, \mathrm{H}-6, \mathrm{H}-4^{\mathrm{B}}\right), 7.45-7.52\left(\mathrm{~m}, 3 \mathrm{H}, \mathrm{H}-3^{\mathrm{B}}, \mathrm{H}-5^{\mathrm{B}}, \mathrm{H}^{-} 4^{\mathrm{E}}\right)$, 7.55-7.62 (m, 2H, H-3 $\left.{ }^{\mathrm{E}}, \mathrm{H}-5^{\mathrm{E}}\right), 7.70(\mathrm{~d}, 1 \mathrm{H}, \mathrm{J}=8.5 \mathrm{~Hz}, \mathrm{H}-8), 7.82-7.91\left(\mathrm{~m}, 5 \mathrm{H}, \mathrm{H}-7, \mathrm{H}-2^{\mathrm{B}}, \mathrm{H}-6^{\mathrm{B}}, \mathrm{H}_{-2}{ }^{\mathrm{E}}\right.$, H-6 $\left.{ }^{\mathrm{E}}\right), 7.98(\mathrm{dd}, 1 \mathrm{H}, J=7.7,1.5 \mathrm{~Hz}, \mathrm{H}-5), 8.75\left(\mathrm{~s}, 1 \mathrm{H}, \mathrm{H}-5^{\mathrm{D}}\right), 8.87$ (s, 1H, H-5 $\left.{ }^{\mathrm{A}}\right)$; ${ }^{13} \mathrm{C} \mathrm{NMR}(126 \mathrm{MHz}$, DMSO- $\left.d_{6}\right) \delta 23.4\left(\mathrm{CH}_{3}\right), 38.7\left(\mathrm{~N}-1-\mathrm{CH}_{2}\right), 73.0(\mathrm{C}-3), 116.8(\mathrm{C}-8), 119.2(\mathrm{C}-4 \mathrm{a}), 120.2\left(\mathrm{C}-2^{\mathrm{E}}, \mathrm{C}-6^{\mathrm{E}}\right), 121.8$ $\left(\mathrm{C}-5^{\mathrm{D}}\right), 122.5\left(\mathrm{C}-5^{\mathrm{A}}\right), 124.0(\mathrm{C}-6), 125.2\left(\mathrm{C}-2^{\mathrm{B}}, \mathrm{C}^{-6^{\mathrm{B}}}\right), 128.1\left(\mathrm{C}-4^{\mathrm{B}}\right), 128.1(\mathrm{C}-5), 128.8\left(\mathrm{C}-4^{\mathrm{E}}\right), 129.1\left(\mathrm{C}-3^{\mathrm{B}}\right.$, C-5 $\left.{ }^{\mathrm{B}}\right), 129.9\left(\mathrm{C}-3^{\mathrm{E}}, \mathrm{C}-5^{\mathrm{E}}\right), 130.6\left(\mathrm{C}-1^{\mathrm{B}}\right), 136.5\left(\mathrm{C}-1^{\mathrm{E}}\right), 137.3(\mathrm{C}-7), 141.6(\mathrm{C}-8 \mathrm{a}), 143.3\left(\mathrm{C}-4^{\mathrm{D}}\right), 146.0\left(\mathrm{C}-4^{\mathrm{A}}\right)$, 168.3 (C-2), $190.0(\mathrm{C}-4) ;{ }^{15} \mathrm{~N}$ NMR (51 MHz, DMSO-d 6 ) $\delta 136.3$ (N1), $248.9\left(\mathrm{~N}-1^{\mathrm{A}}\right), 255.7$ (N-D-1), 347.1 $\left(\mathrm{N}-3^{\mathrm{A}}\right), 353.4\left(\mathrm{~N}-3^{\mathrm{D}}\right), 358.1\left(\mathrm{~N}-2^{\mathrm{D}}\right), 363.2\left(\mathrm{~N}-2^{\mathrm{A}}\right)$; IR $\left(\mathrm{cm}^{-1}\right): v$ 3275, 1721, 1690, 1613, 1485, 1353, 854, 771, 756, 698, 666, 607, 520; MS (EI) $m / z(\%): 476\left(3,[\mathrm{M}+1]^{+}\right), 475\left(8,[\mathrm{M}]^{+}\right), 289(14), 145(12), 131(11)$, 130 (100), 129 (18), 128 (11), 116 (56), 104 (12), 103 (16), 102 (12), 89 (12), 77 (69); HRMS (ESI+): m/z calcd for $\mathrm{C}_{27} \mathrm{H}_{22} \mathrm{~N}_{7} \mathrm{O}_{2}{ }^{+}[\mathrm{M}+\mathrm{H}]^{+}$476.1829, found 476.1825. Anal. Calcd for $\mathrm{C}_{27} \mathrm{H}_{21} \mathrm{~N}_{7} \mathrm{O}_{2}$ (475.50): C, 68.20; H, 4.45; N, 20.62\%. Found: C, 68.48; H, 4.53; N, 20.60\%.

(1-(1-((1-Benzyl-1H-1,2,3-triazol-4-yl)methyl)-3-methyl-2,4-dioxo-1,2,3,4-tetrahydroquinolin-3-yl)-1H1,2,3-triazol-4-yl)methyl acetate (2d). Colorless powder, m.p. $69-82{ }^{\circ} \mathrm{C} ; R_{\mathrm{f}}=0.42(30 \%$ ethyl acetate

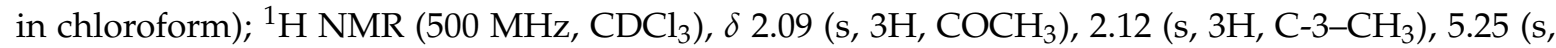
$\left.2 \mathrm{H}, \mathrm{OCH}_{2}\right), 5.33\left(\mathrm{~s}, 2 \mathrm{H}, \mathrm{N}-1-\mathrm{CH}_{2}\right), 5.45\left(\mathrm{~d}, 1 \mathrm{H}, \mathrm{J}=14.8 \mathrm{~Hz}, \mathrm{~N}-1^{\mathrm{D}}-\mathrm{CH} \alpha\right), 5.51(\mathrm{~d}, 1 \mathrm{H}, J=14.8 \mathrm{~Hz}$, N-1 $\left.{ }^{\mathrm{D}}-\mathrm{CH} \beta\right), 7.23-7.26\left(\mathrm{~m}, 3 \mathrm{H}, \mathrm{H}-6, \mathrm{H}-2^{\mathrm{E}}, \mathrm{H}-6^{\mathrm{E}}\right), 7.32-7.38\left(\mathrm{~m}, 3 \mathrm{H}, \mathrm{H}-3^{\mathrm{E}}, \mathrm{H}-4^{\mathrm{E}}, \mathrm{H}-5^{\mathrm{E}}\right), 7.55$ (s, $1 \mathrm{H}$, H-5 $\left.{ }^{\mathrm{D}}\right), 7.73(\mathrm{ddd}, 1 \mathrm{H}, J=8.7,7.1,1.6 \mathrm{~Hz}, \mathrm{H}-7), 7.78\left(\mathrm{~s}, 1 \mathrm{H}, \mathrm{H}-5^{\mathrm{A}}\right), 7.82(\mathrm{~d}, 1 \mathrm{H}, J=8.4 \mathrm{~Hz}, \mathrm{H}-8), 8.02(\mathrm{dd}$, $1 \mathrm{H}, \mathrm{J}=7.7,1.6 \mathrm{~Hz}, \mathrm{H}-5) ;{ }^{13} \mathrm{C} \mathrm{NMR}\left(126 \mathrm{MHz}_{\mathrm{CDCl}}\right) \delta 21.1\left(\mathrm{COCH}_{3}\right), 23.5\left(\mathrm{C}-3-\mathrm{CH}_{3}\right), 39.5\left(\mathrm{~N}-1-\mathrm{CH}_{2}\right)$, $54.5\left(\mathrm{~N}-1^{\mathrm{D}}-\mathrm{CH}_{2}\right), 57.7\left(\mathrm{OCH}_{2}\right), 71.6(\mathrm{C}-3), 116.9(\mathrm{C}-8), 119.2(\mathrm{C}-4 \mathrm{a}), 123.5\left(\mathrm{C}-5^{\mathrm{D}}\right), 124.2\left(\mathrm{C}-5^{\mathrm{A}}\right), 124.6$ (C-6), $128.3\left(\mathrm{C}-2^{\mathrm{E}}, \mathrm{C}-6^{\mathrm{E}}\right), 129.0\left(\mathrm{C}-4^{\mathrm{E}}\right), 129.3\left(\mathrm{C}-3^{\mathrm{E}}, \mathrm{C}-5^{\mathrm{E}}\right), 129.3(\mathrm{C}-5), 134.4\left(\mathrm{C}-1^{\mathrm{E}}\right), 137.8(\mathrm{C}-7), 141.7$ (C-8a), $142.3\left(\mathrm{C}-4^{\mathrm{A}}\right), 142.9\left(\mathrm{C}-4^{\mathrm{D}}\right), 168.2(\mathrm{C}-2), 171.1\left(\mathrm{COCH}_{3}\right), 189.4(\mathrm{C}-4) ;{ }^{15} \mathrm{~N}$ NMR $\left(51 \mathrm{MHz}, \mathrm{CDCl}_{3}\right)$ $\delta 138.7(\mathrm{~N}-1), 248.4\left(\mathrm{~N}-1^{\mathrm{A}}\right), 250.4\left(\mathrm{~N}-1^{\mathrm{D}}\right), 350.0\left(\mathrm{~N}-3^{\mathrm{D}}\right), 355.2\left(\mathrm{~N}-3^{\mathrm{A}}\right), 361.6\left(\mathrm{~N}-2^{\mathrm{A}}\right), 362.6\left(\mathrm{~N}-2^{\mathrm{D}}\right)$; IR $\left(\mathrm{cm}^{-1}\right): v$ 3143, 2930, 1739, 1717, 1679, 1602, 1470, 1384, 1243, 1186, 1050, 1028, 765, 721, 664; MS (EI) $m / z(\%): 486\left(0.3,[\mathrm{M}+1]^{+}\right), 485\left(1,[\mathrm{M}]^{+}\right), 144(18), 91(100), 43(24)$; HRMS (ESI+): $m / z$ calcd for $\mathrm{C}_{25} \mathrm{H}_{24} \mathrm{~N}_{7} \mathrm{O}_{4}{ }^{+}[\mathrm{M}+\mathrm{H}]^{+} 486.1884$, found 486.1884 .

(1-(3-Methyl-2,4-dioxo-1-((1-phenyl-1H-1,2,3-triazol-4-yl)methyl)-1,2,3,4-tetrahydroquinolin-3-yl)-1H1,2,3-triazol-4-yl)methyl acetate (2e). Colorless powder, m.p. $78-97{ }^{\circ} \mathrm{C} ; R_{\mathrm{f}}=0.25$ (30\% ethyl acetate in chloroform); ${ }^{1} \mathrm{H}$ NMR $\left(500 \mathrm{MHz}, \mathrm{CDCl}_{3}\right) \delta 2.10\left(\mathrm{~s}, 3 \mathrm{H}, \mathrm{COCH}_{3}\right), 2.20\left(\mathrm{~s}, 3 \mathrm{H}, \mathrm{C}-3-\mathrm{CH}_{3}\right), 5.27(\mathrm{~s}, 2 \mathrm{H}$, $\left.\mathrm{OCH}_{2}\right), 5.42(\mathrm{~d}, 1 \mathrm{H}, J=15.8 \mathrm{~Hz}, \mathrm{~N}-1-\mathrm{CH} \alpha), 5.52(\mathrm{~d}, 1 \mathrm{H}, J=15.8 \mathrm{~Hz}, \mathrm{~N}-1-\mathrm{CH} \beta), 7.27-7.30(\mathrm{~m}, 1 \mathrm{H}, \mathrm{H}-6)$, 7.41-7.47 (m, 1H, H-4 $\left.{ }^{\mathrm{E}}\right), 7.49-7.55\left(\mathrm{~m}, 2 \mathrm{H}, \mathrm{H}-3^{\mathrm{E}}, \mathrm{H}-5^{\mathrm{E}}\right), 7.69-7.74\left(\mathrm{~m}, 2 \mathrm{H}, \mathrm{H}-2^{\mathrm{E}}, \mathrm{H}-6^{\mathrm{E}}\right), 7.76(\mathrm{ddd}, 1 \mathrm{H}$, $J=8.1,7.7,1.6 \mathrm{~Hz}, \mathrm{H}-7), 7.85(\mathrm{~d}, 1 \mathrm{H}, J=7.3 \mathrm{~Hz}, \mathrm{H}-8), 7.86\left(\mathrm{~s}, 1 \mathrm{H}, \mathrm{H}-5^{\mathrm{A}}\right), 8.05(\mathrm{dd}, 1 \mathrm{H}, J=7.8,1.5 \mathrm{~Hz}$, 
$\mathrm{H}-5), 8.10\left(\mathrm{~s}, 1 \mathrm{H}, \mathrm{H}-5^{\mathrm{D}}\right) ;{ }^{13} \mathrm{C} \mathrm{NMR}\left(126 \mathrm{MHz}, \mathrm{CDCl}_{3}\right) \delta 21.0\left(\mathrm{COCH}_{3}\right), 23.4\left(\mathrm{C}-3-\mathrm{CH}_{3}\right), 39.5\left(\mathrm{~N}-1-\mathrm{CH}_{2}\right)$, $57.7\left(\mathrm{OCH}_{2}\right), 71.5(\mathrm{C}-3), 116.8(\mathrm{C}-8), 119.2(\mathrm{C}-4 \mathrm{a}), 120.6\left(\mathrm{C}-2^{\mathrm{E}}, \mathrm{C}-6^{\mathrm{E}}\right), 121.7\left(\mathrm{C}-5^{\mathrm{D}}\right), 124.1\left(\mathrm{C}-5^{\mathrm{A}}\right), 124.7$ (C-6), $129.1\left(\mathrm{C}-4^{\mathrm{E}}\right), 129.4(\mathrm{C}-5), 129.9\left(\mathrm{C}-3^{\mathrm{E}}, \mathrm{C}-5^{\mathrm{E}}\right), 136.9\left(\mathrm{C}-1^{\mathrm{E}}\right), 137.8(\mathrm{C}-7), 141.7(\mathrm{C}-8 \mathrm{a}), 142.3\left(\mathrm{C}-4^{\mathrm{A}}\right)$, $143.2\left(\mathrm{C}-4^{\mathrm{D}}\right), 168.3(\mathrm{C}-2), 171.1\left(\mathrm{COCH}_{3}\right), 189.4(\mathrm{C}-4) ;{ }^{15} \mathrm{~N} \mathrm{NMR}\left(51 \mathrm{MHz}, \mathrm{CDCl}_{3}\right) \delta 138.7(\mathrm{~N}-1), 248.8$ $\left(\mathrm{N}-1^{\mathrm{A}}\right), 256.3\left(\mathrm{~N}-1^{\mathrm{D}}\right), 351.9\left(\mathrm{~N}-3^{\mathrm{D}}\right), 355.5\left(\mathrm{~N}-3^{\mathrm{A}}\right)$; IR $\left(\mathrm{cm}^{-1}\right): v 3145,2926,1740,1717,1681,1601,1470$, 1384, 1242, 1184, 1046, 761, 691, 664; MS (EI) $m / z$ (\%): $\left.472(0.9 \text {, [M + 1] }]^{+}\right), 471\left(3,[\mathrm{M}]^{+}\right), 303(20), 302$ (17), 131 (13), 130 (100), 129 (14), 77 (44), 43 (25); HRMS (ESI+): $m / z$ calcd for $\mathrm{C}_{24} \mathrm{H}_{22} \mathrm{~N}_{7} \mathrm{O}_{4}{ }^{+}[\mathrm{M}+\mathrm{H}]^{+}$ 472.1728 , found 472.1726 .

1-((1-Benzyl-1H-1,2,3-triazol-4-yl)methyl)-3-phenyl-3-(4-phenyl-1H-1,2,3-triazol-1-yl)quinoline-2,4 (1H,3H)-dione (2g). Colorless crystals, m.p. $142-145{ }^{\circ} \mathrm{C}$ (ethanol); $R_{\mathrm{f}}=0.42$ (30\% ethyl acetate in chloroform); ${ }^{1} \mathrm{H}$ NMR (500 MHz, DMSO- $\left.d_{6}\right) \delta 5.15(\mathrm{~d}, 1 \mathrm{H}, J=15.8 \mathrm{~Hz}, \mathrm{~N}-1-\mathrm{CH} \alpha), 5.62(\mathrm{~s}, 2 \mathrm{H}$, $\left.\mathrm{N}-1^{\mathrm{D}}-\mathrm{CH}_{2}\right), 5.63(\mathrm{~d}, 1 \mathrm{H}, J=15.8 \mathrm{~Hz}, \mathrm{~N}-1-\mathrm{CH} \beta), 7.22-7.50\left(\mathrm{~m}, 14 \mathrm{H}, \mathrm{H}-6, \mathrm{H}-3^{\mathrm{B}}, \mathrm{H}-4^{\mathrm{B}}, \mathrm{H}^{-} 5^{\mathrm{B}}, \mathrm{H}-2^{\mathrm{C}}, \mathrm{H}-3^{\mathrm{C}}\right.$, $\left.\mathrm{H}-4^{\mathrm{C}}, \mathrm{H}-5^{\mathrm{C}}, \mathrm{H}-6^{\mathrm{C}}, \mathrm{H}-2^{\mathrm{E}}, \mathrm{H}-3^{\mathrm{E}}, \mathrm{H}-4^{\mathrm{E}}, \mathrm{H}-5^{\mathrm{E}}, \mathrm{H}-6^{\mathrm{E}}\right), 7.68(\mathrm{~d}, 1 \mathrm{H}, J=7.8 \mathrm{~Hz}, \mathrm{H}-8), 7.73$ (ddd, $1 \mathrm{H}, J=8.5$, 7.1, 1.7 Hz, H-7), 7.80-7.83 (m, 2H, H-2 $\left.{ }^{\mathrm{B}}, \mathrm{H}-6^{\mathrm{B}}\right), 7.92(\mathrm{dd}, 1 \mathrm{H}, J=7.7,1.5 \mathrm{~Hz}, \mathrm{H}-5), 8.24\left(\mathrm{~s}, 1 \mathrm{H}, \mathrm{H}-5^{\mathrm{D}}\right)$, $8.51\left(\mathrm{~s}, 1 \mathrm{H}, \mathrm{H}-5^{\mathrm{A}}\right) ;{ }^{13} \mathrm{C}$ NMR $\left(126 \mathrm{MHz}, \mathrm{DMSO}-d_{6}\right) \delta 39.2\left(\mathrm{~N}-1-\mathrm{CH}_{2}\right), 52.8\left(\mathrm{~N}-1^{\mathrm{D}}-\mathrm{CH}_{2}\right), 80.1(\mathrm{C}-3), 116.7$ (C-8), 120.9 (C-4a), $123.4\left(\mathrm{C}-5^{\mathrm{A}}\right), 124.0(\mathrm{C}-6), 124.2\left(\mathrm{C}-5^{\mathrm{D}}\right), 125.2\left(\mathrm{C}-2^{\mathrm{B}}, \mathrm{C}-6^{\mathrm{B}}\right), 127.9(\mathrm{C}-5), 128.0\left(\mathrm{C}-4^{\mathrm{C}}\right.$, $\left.\mathrm{C}-2^{\mathrm{E}}, \mathrm{C}-6^{\mathrm{E}}\right), 128.2\left(\mathrm{C}-4^{\mathrm{B}}\right), 128.7\left(\mathrm{C}-1^{\mathrm{C}}\right), 128.8\left(\mathrm{C}-3^{\mathrm{E}}, \mathrm{C}-5^{\mathrm{E}}\right), 129.0\left(\mathrm{C}-3^{\mathrm{B}}, \mathrm{C}-5^{\mathrm{B}}\right), 129.4\left(\mathrm{C}-2^{\mathrm{C}}, \mathrm{C}-6^{\mathrm{C}}\right), 129.8$ $\left(\mathrm{C}-1^{\mathrm{B}}\right), 130.5\left(\mathrm{C}-3^{\mathrm{C}}, \mathrm{C}-5^{\mathrm{C}}, \mathrm{C}-4^{\mathrm{E}}\right), 136.0\left(\mathrm{C}-1^{\mathrm{E}}\right), 136.8(\mathrm{C}-7), 140.8(\mathrm{C}-8 \mathrm{a}), 141.9\left(\mathrm{C}-4^{\mathrm{D}}\right), 145.4\left(\mathrm{C}-4^{\mathrm{A}}\right), 166.2$ (C-2), 188.2 (C-4); IR (cm ${ }^{-1}$ ): $v$ 3434, 3138, 3062, 1716, 1678, 1601, 1468, 1375, 1307, 1035, 870, 761, 724, 695; MS (EI) $m / z(\%): 552\left(1,[\mathrm{M}+1]^{+}\right), 551\left(3,[\mathrm{M}]^{+}\right), 289(23), 236(11), 145$ (18), 144 (17), 116 (31), 104 (10), 91 (100), 89 (11), 77 (16); HRMS (ESI+): $m / z$ calcd for $\mathrm{C}_{33} \mathrm{H}_{26} \mathrm{~N}_{7} \mathrm{O}_{2}{ }^{+}[\mathrm{M}+\mathrm{H}]^{+} 552.2142$, found 552.2133. Anal. Calcd for $\mathrm{C}_{33} \mathrm{H}_{25} \mathrm{~N}_{7} \mathrm{O}_{2}$ (551.60): $\mathrm{C}, 71.86 ; \mathrm{H}, 4.57 ; \mathrm{N}, 17.78 \%$. Found: $\mathrm{C}, 71.58 ; \mathrm{H}, 4.58$; $\mathrm{N}, 17.73 \%$.

3-Phenyl-3-(4-phenyl-1H-1,2,3-triazol-1-yl)-1-((1-phenyl-1H-1,2,3-triazol-4-yl)methyl)quinoline-2,4 (1H,3H)-dione (2h). Colorless crystals, m.p. $152-157{ }^{\circ} \mathrm{C}$ (ethanol); $R_{\mathrm{f}}=0.54$ (30\% ethyl acetate in chloroform); ${ }^{1} \mathrm{H}$ NMR (500 MHz, DMSO- $\left.d_{6}\right) \delta 5.33(\mathrm{~d}, 1 \mathrm{H}, J=16.1 \mathrm{~Hz}, \mathrm{~N}-1-\mathrm{CH} \alpha), 5.71(\mathrm{~d}, 1 \mathrm{H}, J=16.1$ $\mathrm{Hz}, \mathrm{N}-1-\mathrm{CH} \beta), 7.25-7.29$ (m, 1H, H-6), 7.32-7.48 (m, 8H, H-3 ${ }^{\mathrm{B}}, \mathrm{H}-4^{\mathrm{B}}, \mathrm{H}-5^{\mathrm{B}}, \mathrm{H}-2^{\mathrm{C}}, \mathrm{H}-3^{\mathrm{C}}, \mathrm{H}-4^{\mathrm{C}}, \mathrm{H}-5^{\mathrm{C}}$, $\left.\mathrm{H}-6^{\mathrm{C}}\right), 7.48-7.53\left(\mathrm{~m}, 1 \mathrm{H}, \mathrm{H}-4^{\mathrm{E}}\right), 7.58-7.64\left(\mathrm{~m}, 2 \mathrm{H}, \mathrm{H}-3^{\mathrm{E}}, \mathrm{H}-5^{\mathrm{E}}\right), 7.70(\mathrm{~d}, 1 \mathrm{H}, J=8.2 \mathrm{~Hz}, \mathrm{H}-8), 7.72-7.77$ (m, 1H, H-7), 7.81-7.85 (m, 2H, H-2 $\left.{ }^{\mathrm{B}}, \mathrm{H}^{\mathrm{B}} \mathrm{6}^{\mathrm{B}}\right), 7.88-7.93\left(\mathrm{~m}, 2 \mathrm{H}, \mathrm{H}-2^{\mathrm{E}}, \mathrm{H}-6^{\mathrm{E}}\right), 7.95(\mathrm{dd}, 1 \mathrm{H}, J=7.7,1.5 \mathrm{~Hz}$, $\mathrm{H}-5), 8.54\left(\mathrm{~s}, 1 \mathrm{H}, \mathrm{H}-5^{\mathrm{A}}\right), 8.83\left(\mathrm{~s}, 1 \mathrm{H}, \mathrm{H}-5^{\mathrm{D}}\right) ;{ }^{13} \mathrm{C}$ NMR $\left(126 \mathrm{MHz}, \mathrm{DMSO}-d_{6}\right) \delta 39.0\left(\mathrm{~N}-1-\mathrm{CH}_{2}\right), 80.3$ (C-3), $116.7(\mathrm{C}-8), 120.2\left(\mathrm{C}-2^{\mathrm{E}}, \mathrm{C}-6^{\mathrm{E}}\right), 120.9(\mathrm{C}-4 \mathrm{a}), 122.3\left(\mathrm{C}-5^{\mathrm{D}}\right), 123.5\left(\mathrm{C}-5^{\mathrm{A}}\right), 124.1(\mathrm{C}-6), 125.2\left(\mathrm{C}-2^{\mathrm{B}}\right.$, C-6 $\left.{ }^{\mathrm{B}}\right), 127.9(\mathrm{C}-5), 128.0\left(\mathrm{C}-4^{\mathrm{B}}\right), 128.9\left(\mathrm{C}-4^{\mathrm{E}}\right), 128.9\left(\mathrm{C}-2^{\mathrm{C}}, \mathrm{C}-6^{\mathrm{C}}\right), 129.0\left(\mathrm{C}-3^{\mathrm{B}}, \mathrm{C}-3^{\mathrm{B}}\right), 129.4\left(\mathrm{C}-3^{\mathrm{C}}, \mathrm{C}-5^{\mathrm{C}}\right)$, $129.9\left(\mathrm{C}-1^{\mathrm{C}}\right), 130.0\left(\mathrm{C}-3^{\mathrm{E}}, \mathrm{C}-5^{\mathrm{E}}\right), 130.5\left(\mathrm{C}-4^{\mathrm{C}}\right), 130.6\left(\mathrm{C}-1^{\mathrm{B}}\right), 136.5\left(\mathrm{C}-1^{\mathrm{E}}\right), 136.8(\mathrm{C}-7), 140.7(\mathrm{C}-8 \mathrm{a}), 142.9$ $\left(\mathrm{C}-4^{\mathrm{D}}\right), 145.4\left(\mathrm{C}-4^{\mathrm{A}}\right), 166.4(\mathrm{C}-2), 188.2(\mathrm{C}-4)$; IR $\left(\mathrm{cm}^{-1}\right): v 3447,3142,3060,1716,1679,1600,1468,1449$, 1375, 1305, 1040, 871, 758, 693; MS (EI) m/z (\%): $538\left(1,[\mathrm{M}+1]^{+}\right), 537\left(3,[\mathrm{M}]^{+}\right), 366(14), 262(10), 236$ (17), 145 (29), 131 (10), 130 (100), 129 (19), 128 (11), 118 (10), 116 (38), 104 (14), 103 (17), 102 (13), 90 (12), 89 (15), 77 (71), 51 (12); HRMS (ESI+): $m / z$ calcd for $\mathrm{C}_{32} \mathrm{H}_{24} \mathrm{~N}_{7} \mathrm{O}_{2}{ }^{+}\left([\mathrm{M}+\mathrm{H}]^{+}\right)$538.1986, found 538.1976. Anal. Calcd for Anal. calcd for $\mathrm{C}_{32} \mathrm{H}_{23} \mathrm{~N}_{7} \mathrm{O}_{2}$ (537.57) C, 71.50; $\mathrm{H}, 4.31 ; \mathrm{N}, 18.24 \%$. Found: $\mathrm{C}, 71.22 ; \mathrm{H}$, $4.32 ; \mathrm{N}, 17.94 \%$.

(1-(1-((1-Benzyl-1H-1,2,3-triazol-4-yl)methyl)-2,4-dioxo-3-phenyl-1,2,3,4-tetrahydroquinolin-3-yl)1H-1,2,3-triazol-4-yl)methyl acetate (2j). Colorless powder, m.p. $188-194{ }^{\circ} \mathrm{C}$ (ethanol); $R_{\mathrm{f}}=0.41(30 \%$ ethyl acetate in chloroform); ${ }^{1} \mathrm{H}$ NMR $\left(500 \mathrm{MHz}, \mathrm{CDCl}_{3}\right) \delta 2.04\left(\mathrm{~s}, 3 \mathrm{H}, \mathrm{CH}_{3}\right), 5.17\left(\mathrm{~s}, 2 \mathrm{H}, \mathrm{OCH}_{2}\right)$, $5.21(\mathrm{~d}, 1 \mathrm{H}, J=15.6 \mathrm{~Hz}, \mathrm{~N}-1-\mathrm{CH} \alpha), 5.43\left(\mathrm{~d}, 1 \mathrm{H}, J=14.8 \mathrm{~Hz}, \mathrm{~N}-1^{\mathrm{D}}-\mathrm{CH} \alpha\right), 5.51(\mathrm{~d}, 1 \mathrm{H}, J=15.6 \mathrm{~Hz}$, $\mathrm{N}-1-\mathrm{CH} \beta), 5.55\left(\mathrm{~d}, 1 \mathrm{H}, J=14.8 \mathrm{~Hz}, \mathrm{~N}-1^{\mathrm{D}}-\mathrm{CH} \beta\right), 7.08\left(\mathrm{~s}, 1 \mathrm{H}, \mathrm{H}-5^{\mathrm{A}}\right), 7.18(\mathrm{ddd}, 1 \mathrm{H}, J=7.5,7.5,0.8 \mathrm{~Hz}$, H-6), 7.23-7.29 (m, 4H, H-3 $\left.{ }^{\mathrm{C}}, \mathrm{H}-5^{\mathrm{C}}, \mathrm{H}-2^{\mathrm{E}}, \mathrm{H}^{\mathrm{E}} \mathrm{6}^{\mathrm{E}}\right), 7.29-7.33$ (m, 2H, H-2 $\left.{ }^{\mathrm{C}}, \mathrm{H}-6^{\mathrm{C}}\right), 7.34-7.39\left(\mathrm{~m}, 3 \mathrm{H}, \mathrm{H}-3^{\mathrm{E}}\right.$, $\left.\mathrm{H}-4^{\mathrm{E}}, \mathrm{H}-5^{\mathrm{E}}\right), 7.38-7.44\left(\mathrm{~m}, 1 \mathrm{H}, \mathrm{H}-4^{\mathrm{C}}\right), 7.58\left(\mathrm{~s}, 1 \mathrm{H}, \mathrm{H}-5^{\mathrm{D}}\right), 7.63(\mathrm{ddd}, 1 \mathrm{H}, J=8.4,7.4,1.7 \mathrm{~Hz}, \mathrm{H}-7), 7.75(\mathrm{~d}$, $1 \mathrm{H}, J=8.3 \mathrm{~Hz}, \mathrm{H}-8), 7.99(\mathrm{dd}, 1 \mathrm{H}, J=7.7,1.7 \mathrm{~Hz}, \mathrm{H}-5) ;{ }^{13} \mathrm{C} \mathrm{NMR}\left(126 \mathrm{MHz}, \mathrm{CDCl}_{3}\right) \delta 21.0\left(\mathrm{CH}_{3}\right), 39.9$ $\left(\mathrm{N}-1-\mathrm{CH}_{2}\right), 54.5\left(\mathrm{~N}-1^{\mathrm{D}}-\mathrm{CH}_{2}\right), 57.6\left(\mathrm{OCH}_{2}\right), 79.6(\mathrm{C}-3), 116.8(\mathrm{C}-8), 120.9(\mathrm{C}-4 \mathrm{a}), 123.5\left(\mathrm{C}-5^{\mathrm{D}}\right), 124.6$ $(\mathrm{C}-6), 126.4\left(\mathrm{C}-5^{\mathrm{A}}\right), 128.3\left(\mathrm{C}-2^{\mathrm{E}}, \mathrm{C}^{\mathrm{E}} \mathrm{E}\right), 128.7\left(\mathrm{C}-2^{\mathrm{C}}, \mathrm{C}-6^{\mathrm{C}}\right), 129.0(\mathrm{C}-5), 129.1\left(\mathrm{C}-4^{\mathrm{E}}\right), 129.4\left(\mathrm{C}-3^{\mathrm{E}}, \mathrm{C}-5^{\mathrm{E}}\right)$, 
$129.7\left(\mathrm{C}-1^{\mathrm{C}}\right), 130.0\left(\mathrm{C}-3^{\mathrm{C}}, \mathrm{C}-5^{\mathrm{C}}\right), 131.3\left(\mathrm{C}-4^{\mathrm{C}}\right), 134.5\left(\mathrm{C}-1^{\mathrm{E}}\right), 137.2(\mathrm{C}-7), 140.9\left(\mathrm{C}-4^{\mathrm{A}}\right), 141.1(\mathrm{C}-8 \mathrm{a}), 142.9$ $\left(\mathrm{C}-4^{\mathrm{D}}\right), 166.6(\mathrm{C}-2), 171.0\left(\mathrm{COCH}_{3}\right), 187.9(\mathrm{C}-4) ;{ }^{15} \mathrm{~N}$ NMR $\left(51 \mathrm{MHz}, \mathrm{CDCl}_{3}\right) \delta 140.4(\mathrm{~N}-1), 249.8\left(\mathrm{~N}-1^{\mathrm{A}}\right)$, $250.4\left(\mathrm{~N}-1^{\mathrm{D}}\right), 350.5\left(\mathrm{~N}-3^{\mathrm{D}}\right), 356.9\left(\mathrm{~N}-3^{\mathrm{A}}\right), 362.9\left(\mathrm{~N}-2^{\mathrm{D}}\right), 365.1\left(\mathrm{~N}-2^{\mathrm{A}}\right) ; \mathrm{IR}\left(\mathrm{cm}^{-1}\right): v$ 3142, 2927, 1740, 1717, 1679, 1602, 1469, 1377, 1244, 768, 749, 714, 697; MS (EI) $m / z(\%): 548\left(0.1,[\mathrm{M}+1]^{+}\right), 547\left(0.3,[\mathrm{M}]^{+}\right), 347$ (13), 289 (13), 144 (14), 105 (10), 104 (13), 91 (100), 43 (29); HRMS (ESI+): $m / z$ calcd for $\mathrm{C}_{30} \mathrm{H}_{26} \mathrm{~N}_{7} \mathrm{O}_{4}{ }^{+}$ $[\mathrm{M}+\mathrm{H}]^{+}$548.2041, found 548.2032.

(1-(2,4-Dioxo-3-phenyl-1-((1-phenyl-1H-1,2,3-triazol-4-yl)methyl)-1,2,3,4-tetrahydroquinolin-3-yl)$1 H-1,2,3-t r i a z o l-4-y l) m e t h y l$ acetate (2k). Colorless powder, m.p. $93-105{ }^{\circ} \mathrm{C} ; R_{\mathrm{f}}=0.42(30 \%$ ethyl acetate in chloroform); ${ }^{1} \mathrm{H}$ NMR $\left(500 \mathrm{MHz}, \mathrm{CDCl}_{3}\right) \delta 2.05\left(\mathrm{~s}, 3 \mathrm{H}, \mathrm{CH}_{3}\right), 5.19\left(\mathrm{~s}, 2 \mathrm{H}, \mathrm{OCH}_{2}\right), 5.42(\mathrm{~d}, 1 \mathrm{H}$, $J=15.7 \mathrm{~Hz}, \mathrm{~N}-1-\mathrm{CH} \alpha), 5.55(\mathrm{~d}, 1 \mathrm{H}, J=15.7 \mathrm{~Hz}, \mathrm{~N}-1-\mathrm{CH} \beta), 7.14\left(\mathrm{~s}, 1 \mathrm{H}, \mathrm{H}-5^{\mathrm{A}}\right), 7.20(\mathrm{ddd}, 1 \mathrm{H}, J=7.6,7.6$, $0.8 \mathrm{~Hz}, \mathrm{H}-6), 7.38-7.49\left(\mathrm{~m}, 6 \mathrm{H}, \mathrm{H}-2^{\mathrm{C}}, \mathrm{H}-3^{\mathrm{C}}, \mathrm{H}-4^{\mathrm{C}}, \mathrm{H}-5^{\mathrm{C}}, \mathrm{H}-6^{\mathrm{C}}, \mathrm{H}-4^{\mathrm{E}}\right), 7.49-7.55\left(\mathrm{~m}, 2 \mathrm{H}, \mathrm{H}-3^{\mathrm{E}}, \mathrm{H}-5^{\mathrm{E}}\right)$, $7.66(\mathrm{ddd}, 1 \mathrm{H}, J=8.5,7.3,1.7 \mathrm{~Hz}, \mathrm{H}-7), 7.68-7.72\left(\mathrm{~m}, 2 \mathrm{H}, \mathrm{H}-2^{\mathrm{E}}, \mathrm{H}-6^{\mathrm{E}}\right), 7.76(\mathrm{~d}, 1 \mathrm{H}, J=8.4 \mathrm{~Hz}, \mathrm{H}-8)$, $8.03(\mathrm{dd}, 1 \mathrm{H}, J=7.8,1.5 \mathrm{~Hz}, \mathrm{H}-5), 8.05\left(\mathrm{~s}, 1 \mathrm{H}, \mathrm{H}-5^{\mathrm{D}}\right) ;{ }^{13} \mathrm{C} \mathrm{NMR}\left(126 \mathrm{MHz}, \mathrm{CDCl}_{3}\right) \delta 21.0\left(\mathrm{CH}_{3}\right), 39.8$ $\left(\mathrm{N}-1-\mathrm{CH}_{2}\right), 57.6\left(\mathrm{OCH}_{2}\right), 79.6(\mathrm{C}-3), 116.7(\mathrm{C}-8), 120.7\left(\mathrm{C}-2^{\mathrm{E}}, \mathrm{C}-6^{\mathrm{E}}\right), 120.9(\mathrm{C}-4 \mathrm{a}), 121.8\left(\mathrm{C}-5^{\mathrm{D}}\right), 124.7$ $(\mathrm{C}-6), 126.4\left(\mathrm{C}-5^{\mathrm{A}}\right), 128.9\left(\mathrm{C}-2^{\mathrm{C}}, \mathrm{C}^{-} \mathrm{C}^{\mathrm{C}}\right), 129.1(\mathrm{C}-5), 129.2\left(\mathrm{C}-4^{\mathrm{E}}\right), 129.9\left(\mathrm{C}-1^{\mathrm{C}}\right), 130.0\left(\mathrm{C}-3^{\mathrm{E}}, \mathrm{C}-5^{\mathrm{E}}\right), 130.2$ $\left(\mathrm{C}-3^{\mathrm{C}}, \mathrm{C}-5^{\mathrm{C}}\right), 131.4\left(\mathrm{C}-4^{\mathrm{C}}\right), 136.9\left(\mathrm{C}-1^{\mathrm{E}}\right), 137.4(\mathrm{C}-7), 140.9\left(\mathrm{C}-4^{\mathrm{A}}\right), 140.9(\mathrm{C}-8 \mathrm{a}), 143.2\left(\mathrm{C}-4^{\mathrm{D}}\right), 166.9(\mathrm{C}-2)$, $171.0\left(\mathrm{COCH}_{3}\right), 187.9(\mathrm{C}-4) ;{ }^{15} \mathrm{~N}$ NMR $\left(51 \mathrm{MHz}, \mathrm{CDCl}_{3}\right) \delta 140.4(\mathrm{~N}-1), 249.9\left(\mathrm{~N}-1^{\mathrm{A}}\right), 256.3\left(\mathrm{~N}-1^{\mathrm{D}}\right), 352.9$ $\left(\mathrm{N}-3^{\mathrm{D}}\right), 357.2\left(\mathrm{~N}-3^{\mathrm{A}}\right) ; \mathrm{IR}\left(\mathrm{cm}^{-1}\right): v$ 3146, 2962, 1741, 1718, 1681, 1600, 1468, 1376, 1243, 1043, 762, 693, 665, 608; MS (EI) $m / z(\%): 534\left(0.2,[\mathrm{M}+1]^{+}\right), 533\left(0.6,[\mathrm{M}]^{+}\right), 366$ (12), 365 (11), 262 (12), 131 (11), 130 (100), 129 (19), 128 (12), 104 (14), 103 (16), 99 (18), 77 (62), 44 (17), 43 (52); HRMS (ESI+): $m / z$ calcd for $\mathrm{C}_{29} \mathrm{H}_{24} \mathrm{~N}_{7} \mathrm{O}_{4}{ }^{+}[\mathrm{M}+\mathrm{H}]^{+}$534.1884, found 534.1882.

3.9. General Procedure for the Synthesis of Bis-Triazoles $2 c, f, i, l$ by Employing $\mathrm{CuSO}_{4} / \mathrm{Cu} u^{0} / \mathrm{DMF}$ Conditions (Table 4, Entries 3, 9, 12 and 15)

A mixture of the appropriate $N$-propargylquinoline-2,4(1H,3H)-dione 7 (1.5 mmol), tetrazolo[1,5-a]pyridine (189 mg, $1.58 \mathrm{mmol}), \mathrm{CuSO}_{4} \cdot 5 \mathrm{H}_{2} \mathrm{O}(38 \mathrm{mg}, 0.15 \mathrm{mmol})$, granular copper (191 mg, $3.05 \mathrm{mmol}$ ) and DMF $(9 \mathrm{~mL})$ was heated in darkness to $95-105^{\circ} \mathrm{C}$ (oil bath) for the time given in Table 4, whereas the color of the mixture changed from brown-black to dark green. The mixture was then allowed to cool to room temperature. Subsequently, $\left(\mathrm{NH}_{4}\right)_{2} \mathrm{CO}_{3}(432 \mathrm{mg}, 4.5 \mathrm{mmol})$ and water $(2 \mathrm{~mL})$ were added and after stirring for $15 \mathrm{~min}$, the mixture was poured into a narrow $(1 \mathrm{~cm}$ diameter) column of silica gel ( $15 \mathrm{~g}$ ). The organic portion was eluted from the column with $10 \%$ ethanol in chloroform. The yellow eluate was washed with saturated aqueous $\mathrm{NH}_{4} \mathrm{Cl}(50 \mathrm{~mL})$, dried over anhydrous sodium sulfate, filtered, and the solvent was removed by rotary evaporation in vacuo. In the cases of $\mathbf{2} \mathbf{c}, \mathbf{i}$, the residue, which was TLC pure compound, was crystallized from suitable solvent. In the cases of $\mathbf{2 f}, \mathbf{l}$, the residue was purified by chromatography on silica gel column using chloroform as eluent. The yields of prepared compounds 2 are given in Table 4 .

3-Methyl-3-(4-phenyl-1H-1,2,3-triazol-1-yl)-1-((1-(pyridin-2-yl)-1H-1,2,3-triazol-4-yl)methyl)quinoline2,4(1H,3H)-dione (2c). Colorless crystals, m.p. $188-191{ }^{\circ} \mathrm{C}$ (benzene); $R_{\mathrm{f}}=0.29$ (30\% ethyl acetate in chloroform); ${ }^{1} \mathrm{H}$ NMR $\left(500 \mathrm{MHz}, \mathrm{DMSO}-d_{6}\right) \delta 2.23\left(\mathrm{~s}, 3 \mathrm{H}, \mathrm{CH}_{3}\right), 5.42(\mathrm{~d}, 1 \mathrm{H}, J=16.5 \mathrm{~Hz}, \mathrm{~N}-1-\mathrm{CH} \alpha)$, $5.58(\mathrm{~d}, 1 \mathrm{H}, J=16.5 \mathrm{~Hz}, \mathrm{~N}-1-\mathrm{CH} \beta), 7.28-7.40\left(\mathrm{~m}, 2 \mathrm{H}, \mathrm{H}-6, \mathrm{H}-4^{\mathrm{B}}\right), 7.43-7.51\left(\mathrm{~m}, 2 \mathrm{H}, \mathrm{H}-3^{\mathrm{B}}, \mathrm{H}-5^{\mathrm{B}}\right)$, 7.51-7.57 (m, 1H, H-5 $\left.{ }^{\mathrm{E}}\right), 7.64(\mathrm{~d}, 1 \mathrm{H}, J=8.4 \mathrm{~Hz}, \mathrm{H}-8), 7.78-7.90\left(\mathrm{~m}, 3 \mathrm{H}, \mathrm{H}-7, \mathrm{H}-2^{\mathrm{B}}, \mathrm{H}-6^{\mathrm{B}}\right), 7.99(\mathrm{~d}, 1 \mathrm{H}$, $J=7.5 \mathrm{~Hz}, \mathrm{H}-5), 8.07-8.17\left(\mathrm{~m}, 2 \mathrm{H}, \mathrm{H}-3^{\mathrm{E}}, \mathrm{H}-4^{\mathrm{E}}\right), 8.54-8.61\left(\mathrm{~m}, 1 \mathrm{H}, \mathrm{H}-6^{\mathrm{E}}\right), 8.82\left(\mathrm{~s}, 1 \mathrm{H}, \mathrm{H}-5^{\mathrm{D}}\right), 8.87(\mathrm{~s}, 1 \mathrm{H}$, $\left.\mathrm{H}-5^{\mathrm{A}}\right) ;{ }^{13} \mathrm{C}$ NMR $\left(126 \mathrm{MHz}, \mathrm{DMSO}-d_{6}\right) \delta 23.4\left(\mathrm{CH}_{3}\right), 38.7\left(\mathrm{~N}-1-\mathrm{CH}_{2}\right), 73.0(\mathrm{C}-3), 113.7\left(\mathrm{C}-3^{\mathrm{E}}\right), 116.6$ (C-8), $119.3(\mathrm{C}-4 \mathrm{a}), 120.6\left(\mathrm{C}-5^{\mathrm{D}}\right), 122.5\left(\mathrm{C}-5^{\mathrm{A}}\right), 123.9(\mathrm{C}-6), 124.5\left(\mathrm{C}-5^{\mathrm{E}}\right), 125.2\left(\mathrm{C}-2^{\mathrm{B}}, \mathrm{C}-6^{\mathrm{B}}\right), 128.1\left(\mathrm{C}-4^{\mathrm{B}}\right)$, $128.1(\mathrm{C}-5), 129.1\left(\mathrm{C}-3^{\mathrm{B}}, \mathrm{C}-5^{\mathrm{B}}\right), 130.5\left(\mathrm{C}-1^{\mathrm{B}}\right), 137.2(\mathrm{C}-7), 140.3\left(\mathrm{C}-4^{\mathrm{E}}\right), 141.4(\mathrm{C}-8 \mathrm{a}), 143.2\left(\mathrm{C}-4^{\mathrm{D}}\right), 146.0$ $\left(\mathrm{C}-4^{\mathrm{A}}\right), 148.4\left(\mathrm{C}-2^{\mathrm{E}}\right), 149.0\left(\mathrm{C}-6^{\mathrm{E}}\right), 168.5(\mathrm{C}-2), 189.9(\mathrm{C}-4) ;{ }^{15} \mathrm{~N}$ NMR $\left(51 \mathrm{MHz}, \mathrm{DMSO}-d_{6}\right) \delta 135.8(\mathrm{~N}-1)$, $248.9\left(\mathrm{~N}-1^{\mathrm{A}}\right), 260.5\left(\mathrm{~N}-1^{\mathrm{D}}\right), 284.9\left(\mathrm{~N}-1^{\mathrm{E}}\right), 347.1\left(\mathrm{~N}-3^{\mathrm{A}}\right), 356.9\left(\mathrm{~N}-3^{\mathrm{D}}\right), 358.6\left(\mathrm{~N}-2^{\mathrm{D}}\right), 363.4\left(\mathrm{~N}-2^{\mathrm{A}}\right) ; \mathrm{IR}$ $\left(\mathrm{cm}^{-1}\right): v$ 3426, 3126, 2972, 1706, 1674, 1601, 1471, 1378, 1310, 1232, 1041, 777, 764; MS (EI) $\mathrm{m} / \mathrm{z}$ (\%): 477 $\left(2,[\mathrm{M}+1]^{+}\right), 476\left(7,[\mathrm{M}]^{+}\right), 289(11), 145(14), 132(14), 131$ (96), 116 (50), 102 (10), 90 (10), 89 (13), 79 (20), 
78 (100), 77 (10), 51 (10); HRMS (ESI+): $m / z$ calcd for $\mathrm{C}_{26} \mathrm{H}_{21} \mathrm{~N}_{8} \mathrm{O}_{2}{ }^{+}[\mathrm{M}+\mathrm{H}]^{+}$477.1782, found 477.1773. Anal. Calcd for $\mathrm{C}_{26} \mathrm{H}_{20} \mathrm{~N}_{8} \mathrm{O}_{2}$ (476.48) $\mathrm{C}, 65.54 ; \mathrm{H}, 4.23 ; \mathrm{N}, 23.52 \%$. Found: $\mathrm{C}, 65.68 ; \mathrm{H}, 4.21 ; \mathrm{N}, 23.63 \%$.

(1-(3-Methyl-2,4-dioxo-1-((1-(pyridin-2-yl)-1H-1,2,3-triazol-4-yl)methyl)-1,2,3,4-tetrahydroquinolin3-yl)-1H-1,2,3-triazol-4-yl)methyl acetate (2f). Colorless powder, m.p. 69-82 ${ }^{\circ} \mathrm{C} ; R_{\mathrm{f}}=0.29(30 \%$ ethyl acetate in chloroform); ${ }^{1} \mathrm{H}$ NMR $\left(500 \mathrm{MHz}, \mathrm{DMSO}-d_{6}\right) \delta 2.06\left(\mathrm{~s}, 3 \mathrm{H}, \mathrm{COCH}_{3}\right), 2.18\left(\mathrm{~s}, 3 \mathrm{H}, \mathrm{C} 3-\mathrm{CH}_{3}\right)$, $5.17(\mathrm{~d}, 1 \mathrm{H}, J=12.7 \mathrm{~Hz}, \mathrm{O}-\mathrm{CH} \alpha), 5.20(\mathrm{~d}, 1 \mathrm{H}, J=12.7 \mathrm{~Hz}, \mathrm{O}-\mathrm{CH} \beta), 5.41(\mathrm{~d}, 1 \mathrm{H}, J=16.5 \mathrm{~Hz}, \mathrm{~N}-1-\mathrm{CH} \alpha)$, $5.53(\mathrm{~d}, 1 \mathrm{H}, J=16.5 \mathrm{~Hz}, \mathrm{~N}-1-\mathrm{CH} \beta), 7.31(\mathrm{dd}, 1 \mathrm{H}, J=7.4,7.4 \mathrm{~Hz}, \mathrm{H}-6), 7.54(\mathrm{dd}, 1 \mathrm{H}, J=8.8,4.5 \mathrm{~Hz}$, $\left.\mathrm{H}-5^{\mathrm{E}}\right), 7.59(\mathrm{~d}, 1 \mathrm{H}, J=8.5 \mathrm{~Hz}, \mathrm{H}-8), 7.77-7.83(\mathrm{~m}, 1 \mathrm{H}, \mathrm{H}-7), 7.96(\mathrm{dd}, 1 \mathrm{H}, J=7.7 \mathrm{~Hz}, J=1.6 \mathrm{~Hz}, \mathrm{H}-5)$, 8.08-8.14 (m, 2H, H-3 $\left.{ }^{\mathrm{E}}, \mathrm{H}-4^{\mathrm{E}}\right), 8.47\left(\mathrm{~s}, 1 \mathrm{H}, \mathrm{H}-5^{\mathrm{A}}\right), 8.55-8.59\left(\mathrm{~m}, 1 \mathrm{H}, \mathrm{H}-6^{\mathrm{E}}\right), 8.82\left(\mathrm{~s}, 1 \mathrm{H}, \mathrm{H}-5^{\mathrm{D}}\right) ;{ }^{13} \mathrm{C}$ NMR $\left(126 \mathrm{MHz}, \mathrm{DMSO}-d_{6}\right) \delta 20.6\left(\mathrm{COCH}_{3}\right), 23.5\left(\mathrm{C} 3-\mathrm{CH}_{3}\right), 38.7\left(\mathrm{~N}-1-\mathrm{CH}_{2}\right), 57.2\left(\mathrm{OCH}_{2}\right), 73.3(\mathrm{C}-3), 113.7$ $\left(\mathrm{C}-3^{\mathrm{E}}\right), 116.5(\mathrm{C}-8), 119.4(\mathrm{C}-4 \mathrm{a}), 120.6\left(\mathrm{C}-5^{\mathrm{D}}\right), 123.8(\mathrm{C}-6), 124.4\left(\mathrm{C}-5^{\mathrm{E}}\right), 126.1\left(\mathrm{C}-5^{\mathrm{A}}\right), 127.9(\mathrm{C}-5), 137.0$ $(\mathrm{C}-7), 140.2\left(\mathrm{C}-4^{\mathrm{E}}\right), 141.3(\mathrm{C}-8 \mathrm{a}), 141.6\left(\mathrm{C}-4^{\mathrm{A}}\right), 143.2\left(\mathrm{C}-4^{\mathrm{D}}\right), 148.3\left(\mathrm{C}-2^{\mathrm{E}}\right), 148.9\left(\mathrm{C}-6^{\mathrm{E}}\right), 168.6(\mathrm{C}-2), 170.1$ $\left(\mathrm{COCH}_{3}\right), 189.9(\mathrm{C}-4) ;{ }^{15} \mathrm{~N}$ NMR $\left(51 \mathrm{MHz}, \mathrm{DMSO}-d_{6}\right) \delta 135.3(\mathrm{~N} 1), 247.6\left(\mathrm{~N}-1^{\mathrm{A}}\right), 260.0\left(\mathrm{~N}-1^{\mathrm{D}}\right), 284.7$ $\left(\mathrm{N}-1^{\mathrm{E}}\right), 353.4\left(\mathrm{~N}-3^{\mathrm{A}}\right), 356.5\left(\mathrm{~N}-3^{\mathrm{D}}\right), 361.9\left(\mathrm{~N}-2^{\mathrm{D}}\right), 363.7\left(\mathrm{~N}-2^{\mathrm{A}}\right)$; IR $\left(\mathrm{cm}^{-1}\right): v 3152,1741,1718$ 1681, 1600, $1471,1384,1314,1242,1183,1038,782,756,663$; MS (EI) $m / z(\%): 473\left(0.7,[\mathrm{M}+1]^{+}\right), 472\left(2,[\mathrm{M}]^{+}\right)$, 304 (27), 303 (26), 302 (17), 132 (13), 131 (100), 79 (22), 78 (100), 43 (21); HRMS (ESI+): $m / z$ calcd for $\mathrm{C}_{23} \mathrm{H}_{21} \mathrm{~N}_{8} \mathrm{O}_{4}{ }^{+}[\mathrm{M}+\mathrm{H}]^{+}$473.1680, found 473.1684. Anal. Calcd for $\mathrm{C}_{23} \mathrm{H}_{20} \mathrm{~N}_{8} \mathrm{O}_{4} \cdot \frac{1}{2} \mathrm{H}_{2} \mathrm{O}$ (472.46): $\mathrm{C}$, $57.38 ; \mathrm{H}, 4.40 ; \mathrm{N}, 23.27 \%$. Found: $\mathrm{C}, 57.39 ; \mathrm{H}, 4.36 ; \mathrm{N}, 23.47 \%$.

3-Phenyl-3-(4-phenyl-1H-1,2,3-triazol-1-yl)-1-((1-(pyridin-2-yl)-1H-1,2,3-triazol-4-yl)methyl)quinoline2,4(1H,3H)-dione (2i). Colorless crystals, m.p. $188-192{ }^{\circ} \mathrm{C}$ (benzene); $R_{\mathrm{f}}=0.50$ (30\% ethyl acetate in chloroform); ${ }^{1} \mathrm{H}$ NMR $\left(500 \mathrm{MHz}, \mathrm{DMSO}-d_{6}\right) \delta 5.44(\mathrm{~d}, 1 \mathrm{H}, J=16.3 \mathrm{~Hz}, \mathrm{~N}-1-\mathrm{CH} \alpha), 5.67(\mathrm{~d}, 1 \mathrm{H}$, $J=16.3 \mathrm{~Hz}, \mathrm{~N}-1-\mathrm{CH} \beta), 7.26(\mathrm{dd}, 1 \mathrm{H}, J=7.5,7.5 \mathrm{~Hz}, \mathrm{H}-6), 7.32-7.40\left(\mathrm{~m}, 3 \mathrm{H}, \mathrm{H}-4^{\mathrm{B}}, \mathrm{H}-2^{\mathrm{C}}, \mathrm{H}-6^{\mathrm{C}}\right)$, 7.41-7.52 (m, 5H, H-3 $\left.3^{\mathrm{B}}, \mathrm{H}-5^{\mathrm{B}}, \mathrm{H}-3^{\mathrm{C}},{\mathrm{H}-4^{\mathrm{C}}}^{\mathrm{C}}, \mathrm{H}-5^{\mathrm{C}}\right), 7.52-7.61\left(\mathrm{~m}, 2 \mathrm{H}, \mathrm{H}-8, \mathrm{H}-5^{\mathrm{E}}\right), 7.68-7.75$ (m, $\left.1 \mathrm{H}, \mathrm{H}-7\right)$, 7.78-7.86 (m, 2H, H-2 $\left.{ }^{\mathrm{B}}, \mathrm{H}-6^{\mathrm{B}}\right), 7.96(\mathrm{dd}, 1 \mathrm{H}, J=7.7,1.5 \mathrm{~Hz}, \mathrm{H}-5), 8.09-8.16\left(\mathrm{~m}, 2 \mathrm{H}, \mathrm{H}-3^{\mathrm{E}}, \mathrm{H}-4^{\mathrm{E}}\right), 8.58(\mathrm{~s}$, $\left.1 \mathrm{H}, \mathrm{H}-5^{\mathrm{A}}\right), 8.60\left(\mathrm{ddd}, 1 \mathrm{H}, J=4.8,1.3,1.3 \mathrm{~Hz}, \mathrm{H}-6^{\mathrm{E}}\right), 8.81\left(\mathrm{~s}, 1 \mathrm{H}, \mathrm{H}-5^{\mathrm{D}}\right) ;{ }^{13} \mathrm{C}$ NMR $\left(126 \mathrm{MHz}, \mathrm{DMSO}-d_{6}\right)$ $\delta 39.1\left(\mathrm{~N}-1-\mathrm{CH}_{2}\right), 80.4(\mathrm{C}-3), 113.7\left(\mathrm{C}-3^{\mathrm{E}}\right), 116.5(\mathrm{C}-8), 120.8\left(\mathrm{C}-5^{\mathrm{D}}\right), 120.9(\mathrm{C}-4 \mathrm{a}), 123.4\left(\mathrm{C}-5^{\mathrm{A}}\right), 124.0$ $(\mathrm{C}-6), 124.5\left(\mathrm{C}-5^{\mathrm{E}}\right), 125.2\left(\mathrm{C}-2^{\mathrm{B}}, \mathrm{C}^{-} 6^{\mathrm{B}}\right), 127.9(\mathrm{C}-5), 128.0\left(\mathrm{C}-4^{\mathrm{B}}\right), 128.9\left(\mathrm{C}-2^{\mathrm{C}}, \mathrm{C}-6^{\mathrm{C}}\right), 129.0\left(\mathrm{C}-3^{\mathrm{B}}, \mathrm{C}-5^{\mathrm{B}}\right)$, $129.4\left(\mathrm{C}-3^{\mathrm{C}}, \mathrm{C}-5^{\mathrm{C}}\right), 130.0\left(\mathrm{C}-1^{\mathrm{C}}\right), 130.5\left(\mathrm{C}-4^{\mathrm{C}}\right), 130.6\left(\mathrm{C}-1^{\mathrm{B}}\right), 136.8(\mathrm{C}-7), 140.2\left(\mathrm{C}-4^{\mathrm{E}}\right), 140.5(\mathrm{C}-8 \mathrm{a}), 143.0$ $\left(\mathrm{C}-4^{\mathrm{D}}\right), 145.4\left(\mathrm{C}-4^{\mathrm{A}}\right), 148.3\left(\mathrm{C}-2^{\mathrm{E}}\right), 149.0\left(\mathrm{C}-6^{\mathrm{E}}\right), 166.6(\mathrm{C}-2), 188.1(\mathrm{C}-4) ;{ }^{15}$ N NMR $\left(51 \mathrm{MHz}, \mathrm{DMSO}-d_{6}\right) \delta$ $137.5(\mathrm{~N} 1), 248.7\left(\mathrm{~N}-1^{\mathrm{A}}\right), 260.4\left(\mathrm{~N}-1^{\mathrm{D}}\right), 284.8\left(\mathrm{~N}-1^{\mathrm{E}}\right), 347.2\left(\mathrm{~N}-3^{\mathrm{A}}\right), 357.7\left(\mathrm{~N}-3^{\mathrm{D}}\right), 367.4\left(\mathrm{~N}-2^{\mathrm{A}}\right) ; \mathrm{IR}\left(\mathrm{cm}^{-1}\right)$ : $v$ 3418, 2973, 1718, 1679, 1596, 1477, 1467, 1450, 1049, 1031, 773, 766, 757, 701; MS (EI) $m / z$ (\%): 539 (1, $\left.[\mathrm{M}+1]^{+}\right), 538\left(3,[\mathrm{M}]^{+}\right), 236(11), 145(16), 132(14), 131(100), 116$ (32), 91 (11), 89 (11), 79 (15), 78 (85), 77 (13); HRMS (ESI+): $m / z$ calcd for $\mathrm{C}_{31} \mathrm{H}_{23} \mathrm{~N}_{8} \mathrm{O}_{2}{ }^{+}[\mathrm{M}+\mathrm{H}]^{+} 539.1938$, found 539.1932. Anal. calcd for $\mathrm{C}_{31} \mathrm{H}_{22} \mathrm{~N}_{8} \mathrm{O}_{2}$ (538.19): $\mathrm{C}, 69.13 ; \mathrm{H}, 4.12 ; \mathrm{N}, 20.81 \%$. Found: $\mathrm{C}, 68.91 ; \mathrm{H}, 4.17 ; \mathrm{N}, 20.66 \%$.

(1-(2,4-Dioxo-3-phenyl-1-((1-(pyridin-2-yl)-1H-1,2,3-triazol-4-yl)methyl)-1,2,3,4-tetrahydroquinolin3-yl)-1H-1,2,3-triazol-4-yl)methyl acetate (21). Colorless powder, m.p. 93-102 ${ }^{\circ} \mathrm{C} ; R_{\mathrm{f}}=0.18(30 \%$ ethyl acetate in chloroform); ${ }^{1} \mathrm{H}$ NMR $\left(500 \mathrm{MHz}, \mathrm{CDCl}_{3}\right) \delta 2.05\left(\mathrm{~s}, 3 \mathrm{H}, \mathrm{COCH}_{3}\right), 5.19\left(\mathrm{~s}, 2 \mathrm{H}, \mathrm{OCH}_{2}\right), 5.30(\mathrm{~d}$, $1 \mathrm{H}, J=15.8 \mathrm{~Hz}, \mathrm{~N}-1-\mathrm{CH} \alpha), 5.71(\mathrm{~d}, 1 \mathrm{H}, J=15.8 \mathrm{~Hz}, \mathrm{~N}-1-\mathrm{CH} \beta), 7.13\left(\mathrm{~s}, 1 \mathrm{H}, \mathrm{H}-5^{\mathrm{A}}\right), 7.19(\mathrm{dd}, 1 \mathrm{H}, J=7.5$, $7.5 \mathrm{~Hz}, \mathrm{H}-6), 7.36\left(\mathrm{dd}, 1 \mathrm{H}, J=7.3,4.9 \mathrm{~Hz}, \mathrm{H}-5^{\mathrm{E}}\right), 7.38-7.42\left(\mathrm{~m}, 2 \mathrm{H}, \mathrm{H}-3^{\mathrm{C}}, \mathrm{H}-5^{\mathrm{C}}\right), 7.42-7.48\left(\mathrm{~m}, 3 \mathrm{H}, \mathrm{H}-2^{\mathrm{C}}\right.$, $\left.\mathrm{H}-4^{\mathrm{C}}, \mathrm{H}-6^{\mathrm{C}}\right), 7.63(\mathrm{ddd}, 1 \mathrm{H}, J=8.3,7.4,1.6 \mathrm{~Hz}, \mathrm{H}-7), 7.70(\mathrm{~d}, 1 \mathrm{H}, J=8.4 \mathrm{~Hz}, \mathrm{H}-8), 7.88-7.95(\mathrm{~m}, 1 \mathrm{H}$, $\left.\mathrm{H}-4^{\mathrm{E}}\right), 8.02(\mathrm{dd}, 1 \mathrm{H}, J=7.8,1.5 \mathrm{~Hz}, \mathrm{H}-5), 8.15\left(\mathrm{~d}, 1 \mathrm{H}, J=8.2 \mathrm{~Hz}, \mathrm{H}-3^{\mathrm{E}}\right), 8.47-8.53\left(\mathrm{~m}, 1 \mathrm{H}, \mathrm{H}-6^{\mathrm{E}}\right), 8.63$ $\left(\mathrm{s}, 1 \mathrm{H}, \mathrm{H}-5^{\mathrm{D}}\right) ;{ }^{13} \mathrm{C}$ NMR $\left(126 \mathrm{MHz}, \mathrm{CDCl}_{3}\right) \delta 21.0\left(\mathrm{COCH}_{3}\right), 39.9\left(\mathrm{~N}-1-\mathrm{CH}_{2}\right), 57.6\left(\mathrm{OCH}_{2}\right), 79.7(\mathrm{C}-3)$, $113.9\left(\mathrm{C}-3^{\mathrm{E}}\right), 116.6(\mathrm{C}-8), 121.0(\mathrm{C}-4 \mathrm{a}), 121.0\left(\mathrm{C}-5^{\mathrm{D}}\right), 124.0\left(\mathrm{C}-5^{\mathrm{E}}\right), 124.6(\mathrm{C}-6), 126.4\left(\mathrm{C}-5^{\mathrm{A}}\right), 128.9\left(\mathrm{C}-2^{\mathrm{C}}\right.$, C-6 $\left.{ }^{\mathrm{C}}\right), 129.1(\mathrm{C}-5), 129.7\left(\mathrm{C}-1^{\mathrm{C}}\right), 130.2\left(\mathrm{C}-3^{\mathrm{C}}, \mathrm{C}^{-5^{\mathrm{C}}}\right), 131.3\left(\mathrm{C}-4^{\mathrm{C}}\right), 137.2(\mathrm{C}-7), 139.3\left(\mathrm{C}-4^{\mathrm{E}}\right), 140.9\left(\mathrm{C}-4^{\mathrm{A}}\right)$, 141.2 (C-8a), $143.0\left(\mathrm{C}-4^{\mathrm{D}}\right), 148.9\left(\mathrm{C}-6^{\mathrm{E}}\right), 149.0\left(\mathrm{C}-2^{\mathrm{E}}\right), 166.6(\mathrm{C}-2), 171.0\left(\mathrm{COCH}_{3}\right), 187.9(\mathrm{C}-4) ;{ }^{15} \mathrm{~N}$ NMR $\left(51 \mathrm{MHz}, \mathrm{CDCl}_{3}\right) \delta 138.9(\mathrm{~N}-1), 249.7\left(\mathrm{~N}-1^{\mathrm{A}}\right), 261.2\left(\mathrm{~N}-1^{\mathrm{D}}\right), 285.1\left(\mathrm{~N}-1^{\mathrm{E}}\right), 355.8\left(\mathrm{~N}-3^{\mathrm{D}}\right), 357.1\left(\mathrm{~N}-3^{\mathrm{A}}\right) ; \mathrm{IR}$ $\left(\mathrm{cm}^{-1}\right): v$ 3155, 2926, 1741, 1718, 1682, 1599, 1470, 1375, 1313, 1243, 1034, 779, 697, 665; MS (EI) $\mathrm{m} / \mathrm{z}(\%)$ : $535\left(0.4,[\mathrm{M}+1]^{+}\right), 534\left(0.7,[\mathrm{M}]^{+}\right), 132$ (14), 131 (100), 79 (19), 78 (93), 44 (11), 43 (31); HRMS (ESI+): $m / z$ calcd for $\mathrm{C}_{28} \mathrm{H}_{23} \mathrm{~N}_{8} \mathrm{O}_{4}{ }^{+}[\mathrm{M}+\mathrm{H}]^{+} 535.1837$, found 535.1846. 
3.10. Synthesis of Bis-Triazole $2 d$ by Employing $\mathrm{CH}_{2} \mathrm{Cl}_{2} /$ Water $/ \mathrm{CuSO}_{4} \cdot 5 \mathrm{H}_{2} \mathrm{O} / \mathrm{Na}$-Ascorbate Conditions (Table 4, Entry 5)

To a solution of acetylene $7 \mathrm{c}(132 \mathrm{mg}, 0.375 \mathrm{mmol})$ and azide 8a (52.4 $\mathrm{mg}, 0.394 \mathrm{mmol})$ in dichloromethane $(6.5 \mathrm{~mL})$ a solution of sodium ascorbate $(59.5 \mathrm{mg}, 0.3 \mathrm{mmol})$ in water $(5.5 \mathrm{~mL})$, and a solution of $\mathrm{CuSO}_{4} \cdot 5 \mathrm{H}_{2} \mathrm{O}(7.5 \mathrm{mg}, 0.03 \mathrm{mmol})$ in water $(1 \mathrm{~mL})$ were added. The two-phase liquid reaction mixture was stirred in darkness at room temperature until the compound $7 \mathrm{c}$ reacted completely according to TLC analysis $(4 \mathrm{~h})$. The reaction mixture was diluted with water $(50 \mathrm{~mL})$ and extracted with chloroform $(4 \times 30 \mathrm{~mL})$. The combined organic layers were dried $\left(\mathrm{Na}_{2} \mathrm{SO}_{4}\right)$, filtered, and evaporated to dryness. The residue was dissolved in chloroform $(5 \mathrm{~mL})$ and subjected to silica gel ( $25 \mathrm{~g}$ ) column chromatography using 67\% ethyl acetate in petroleum ether as eluent, affording product 2d (155 mg, $0.32 \mathrm{mmol}, 85 \%)$.

\subsection{Synthesis of Bis-Triazole $2 d$ by Employing $t$-BuOH/Water $/ \mathrm{CH}_{3} \mathrm{CN} / \mathrm{CuSO} \mathrm{S}_{4} \cdot 5 \mathrm{H}_{2} \mathrm{O} / \mathrm{Na}$-Ascorbate Conditions (Table 4, Entry 6)}

To a mixture of acetylene $7 \mathrm{c}(264 \mathrm{mg}, 0.75 \mathrm{mmol})$, azide $8 \mathrm{a}(105 \mathrm{mg}, 0.79 \mathrm{mmol})$ and $t$ - $\mathrm{BuOH}$ $(3.5 \mathrm{~mL})$ a solution of Na-ascorbate $(30 \mathrm{mg}, 0.15 \mathrm{mmol})$ in water $(2.5 \mathrm{~mL})$, and a solution of $\mathrm{CuSO}_{4} \cdot 5 \mathrm{H}_{2} \mathrm{O}$ $(4 \mathrm{mg}, 0.02 \mathrm{mmol})$ in water $(1 \mathrm{~mL})$ were added. The reaction mixture was stirred in darkness at room temperature for $9 \mathrm{~h}$. Then a solution of Na-ascorbate $(89 \mathrm{mg}, 0.45 \mathrm{mmol})$ in water $(1 \mathrm{~mL})$, and a solution of $\mathrm{CuSO}_{4} \cdot 5 \mathrm{H}_{2} \mathrm{O}(11 \mathrm{mg}, 0.044 \mathrm{mmol})$ in water $(1 \mathrm{~mL})$ and $t$-BuOH $(2 \mathrm{~mL})$ were added. The reaction mixture was stirred for additional $20 \mathrm{~h}$. The resulting sticky sediment that formed in the course of the reaction was dissolved by addition of acetonitrile $(3 \mathrm{~mL})$ to the reaction mixture. The reaction mixture was stirred for additional $19 \mathrm{~h}$. Although the azide and acetylene coupling partners were still present in the reaction mixture, as judged by TLC analysis, the reaction was stopped by the addition of water $(50 \mathrm{~mL})$ and extracted with chloroform $(4 \times 30 \mathrm{~mL})$. The combined organic layers were dried $\left(\mathrm{Na}_{2} \mathrm{SO}_{4}\right)$, filtered, and evaporated to dryness. The residue was dissolved in chloroform and subjected to silica gel (35 g) column chromatography using 67\% ethyl acetate in petroleum ether as eluent, affording regenerated starting acetylene $7 \mathrm{c}(48 \mathrm{mg}, 0.14 \mathrm{mmol}, 18 \%)$ and product $2 \mathrm{~d}(295 \mathrm{mg}, 0.61 \mathrm{mmol}, 81 \%)$.

\subsection{Synthesis of Bis-Triazole $2 d$ by Employing $t$-BuOH/Water $/ \mathrm{CuSO} \mathrm{S}_{4} \cdot 5 \mathrm{H}_{2} \mathrm{O} / \mathrm{L}$-Ascorbic Acid Conditions (Table 4, Entry 7)}

To a mixture of acetylene 7c (264 $\mathrm{mg}, 0.75 \mathrm{mmol})$ and azide $8 \mathrm{a}(105 \mathrm{mg}, 0.79 \mathrm{mmol})$ a solution of L-ascorbic acid (13 mg, $0.074 \mathrm{mmol})$ and $\mathrm{CuSO}_{4} .5 \mathrm{H}_{2} \mathrm{O}(2 \mathrm{mg}, 0.008 \mathrm{mmol})$ in water $(3.5 \mathrm{~mL})$, and $t-\mathrm{BuOH}(3.5 \mathrm{~mL})$ were added. The reaction mixture was stirred in darkness at room temperature. After $8.5 \mathrm{~h}$ and $22 \mathrm{~h}$ of stirring additional portions of L-ascorbic acid $/ \mathrm{CuSO}_{4} \cdot 5 \mathrm{H}_{2} \mathrm{O} / \mathrm{water} / \mathrm{t}$ - $\mathrm{BuOH}$ ( $40 \mathrm{mg}, 0.23 \mathrm{mmol} / 6 \mathrm{mg}, 0.02 \mathrm{mmol} / 1 \mathrm{~mL} / 1 \mathrm{~mL}$ and $53 \mathrm{mg}, 0.3 \mathrm{mmol} / 7.5 \mathrm{mg}, 0.03 \mathrm{mmol} / 1 \mathrm{~mL} / 1 \mathrm{~mL}$, respectively) were added. Although after stirring for additional $23 \mathrm{~h}$ (total reaction time $45 \mathrm{~h}$ ), TLC analysis indicated the presence of azide and acetylene starting compounds, the heterogeneous reaction mixture (a sticky sediment was formed) was diluted with water and extracted with chloroform $(5 \times 50 \mathrm{~mL})$. The combined organic layers were dried $\left(\mathrm{Na}_{2} \mathrm{SO}_{4}\right)$, filtered, and evaporated to dryness. The residue was dissolved in chloroform (5 mL) and subjected to silica gel (35 g) column chromatography using 33\% ethyl acetate in petroleum ether as eluent, affording regenerated starting acetylene 7c (114 mg, $0.32 \mathrm{mmol}, 43 \%)$ and product $2 \mathrm{~d}(165 \mathrm{mg}, 0.34 \mathrm{mmol} 45 \%)$.

\subsection{3-Azido-3-methyl-1-(prop-2-yn-1-yl)Quinoline-2,4(1H,3H)-Dione (9a) (Scheme 3)}

A mixture of the azide $5 \mathrm{a}(649 \mathrm{mg}, 3.0 \mathrm{mmol})$ and potassium carbonate $(1.24 \mathrm{~g}, 9 \mathrm{mmol})$ in DMF $(15 \mathrm{~mL})$ was stirred at room temperature in darkness for $40 \mathrm{~min}$. Propargyl bromide $(6 \mathbf{c}, 80 \%$ solution in toluene, $669 \mathrm{mg}$, $4.5 \mathrm{mmol})$ diluted with DMF $(7 \mathrm{~mL})$ was added dropwise under stirring during $1 \mathrm{~min}$. The reaction mixture was stirred for $6 \mathrm{~h}$, during which time it turned yellow, diluted with cold water $(200 \mathrm{~mL})$ and extracted with chloroform $(5 \times 50 \mathrm{~mL})$. The combined organic layers were dried $\left(\mathrm{Na}_{2} \mathrm{SO}_{4}\right)$, filtered, and evaporated to dryness. Trace amounts of DMF were removed by five 
subsequent co-destilations in vacuo at $50{ }^{\circ} \mathrm{C}$ with toluene $(30 \mathrm{~mL})$. The residual yellow oil was dissolved in chloroform ( $5 \mathrm{~mL}$ ) and chromatographed on column silica gel (35 g) using chloroform as eluent, affording product 9a $(717 \mathrm{mg}, 2.82 \mathrm{mmol}, 94 \%$, dried in vacuo to constant weight) as off-white oily material, that was pure by TLC $\left(R_{\mathrm{f}}=0.57\right.$; chloroform $) ;{ }^{1} \mathrm{H} \mathrm{NMR}\left(500 \mathrm{MHz}, \mathrm{CDCl}_{3}\right) \delta 1.79(\mathrm{~s}, 3 \mathrm{H}), 2.29$ $(\mathrm{dd}, 1 \mathrm{H}, J=2.5,2.5 \mathrm{~Hz}), 4.67(\mathrm{dd}, 1 \mathrm{H}, J=17.8,2.5 \mathrm{~Hz}), 4.98(\mathrm{dd}, 1 \mathrm{H}, J=17.8,2.5 \mathrm{~Hz}), 7.26(\mathrm{ddd}, 1 \mathrm{H}$, $J=7.7,7.4,0.8 \mathrm{~Hz}), 7.35(\mathrm{~d}, 1 \mathrm{H}, J=8.3 \mathrm{~Hz}), 7.71(\mathrm{ddd}, 1 \mathrm{H}, J=8.3,7.4,1.7 \mathrm{~Hz}), 8.02(\mathrm{dd}, 1 \mathrm{H}, J=7.7$, $1.7 \mathrm{~Hz}) ;{ }^{13} \mathrm{C}$ NMR $\left(126 \mathrm{MHz}, \mathrm{CDCl}_{3}\right) \delta 23.6,32.7,70.7,73.4,77.1,115.8,119.6,124.4,129.0,136.9,140.8$, 169.1, 191.1; IR (cm $\left.{ }^{-1}\right): v$ 3241, 2980, 2138, 2107, 1711, 1678, 1603, 1471, 1383, 1366, 1305, 1285, 1260, 1218, 762; HRMS (ESI+): $m / z$ calcd for $\mathrm{C}_{13} \mathrm{H}_{11} \mathrm{~N}_{4} \mathrm{O}_{2}{ }^{+}[\mathrm{M}+\mathrm{H}]^{+}$255.0877, found 255.0877; calcd for $\mathrm{C}_{13} \mathrm{H}_{11} \mathrm{~N}_{2} \mathrm{O}_{2}{ }^{+}\left[\mathrm{M}-\mathrm{N}_{2}+\mathrm{H}\right]^{+}$227.0815, found 227.0814.

\subsection{Synthesis of Triazole 7a from Phenylacetylene (6a) and Compound 9a (Scheme 3)}

A mixture of compound 9a (286 mg, $1.13 \mathrm{mmol})$, phenylacetylene (6a) (230 mg, $2.25 \mathrm{mmol})$, $\mathrm{CuSO}_{4} 5 \mathrm{H}_{2} \mathrm{O}(28 \mathrm{mg}, 0.11 \mathrm{mmol})$ and granular copper (143 mg, $\left.2.25 \mathrm{mmol}\right)$ in DMF (5 mL) was stirred in darkness at room temperature for $60 \mathrm{~min}$. To the resulting brown-green suspension $\left(\mathrm{NH}_{4}\right)_{2} \mathrm{CO}_{3}$ $(324 \mathrm{mg}, 3.38 \mathrm{mmol})$ and water $(3 \mathrm{~mL})$ were added and stirring was continued for $10 \mathrm{~min}$. The resulting mixture was diluted with $10 \%$ ethanol in chloroform $(10 \mathrm{~mL})$. The organic layer was separated and the aqueous layer was extracted with chloroform $(3 \times 10 \mathrm{~mL})$. The combined organic layers were passed through a narrow $(1 \mathrm{~cm}$ in diameter) column of silica gel $(13 \mathrm{~g})$ and the column was subsequently washed with $10 \%$ ethanol in chloroform $(210 \mathrm{~mL})$ using overpressure to the top of the column. The yellow eluate was washed with saturated aqueous $\mathrm{NH}_{4} \mathrm{Cl}(1 \times 50 \mathrm{~mL})$ and distilled water $(1 \times 50 \mathrm{~mL})$, dried $\left(\mathrm{Na}_{2} \mathrm{SO}_{4}\right)$, filtered, and evaporated to dryness. Trace amounts of DMF were removed by five subsequent co-destilations in vacuo at $50{ }^{\circ} \mathrm{C}$ with toluene $(40 \mathrm{~mL})$. The residue was chromatographed on a column of silica gel (30 g) using 38\% ethyl acetate in hexane. The resulting white solid (88 mg) was crystallized from benzene affording triazole $7 \mathrm{a}$ (66 mg, $0.19 \mathrm{mmol}, 16 \%)$, which was identified with the compound $7 \mathbf{a}$ described above.

\subsection{3-Azido-1-((1-Benzyl-1H-1,2,3-Triazol-4-yl)Methyl)-3-Methylquinoline-2,4(1H,3H)-Dione (10a)} (Scheme 3)

A mixture of acetylene 9a (254 mg, $1.0 \mathrm{mmol})$, (azidomethyl)benzene (8a) (266 mg, $2.0 \mathrm{mmol})$, $\mathrm{CuSO}_{4} \cdot 5 \mathrm{H}_{2} \mathrm{O}(25 \mathrm{mg}, 0.1 \mathrm{mmol})$ and granular copper $(127 \mathrm{mg}, 2.0 \mathrm{mmol})$ in DMF $(10 \mathrm{~mL})$ was stirred at room temperature for $21 \mathrm{~h}$. Then $\left(\mathrm{NH}_{4}\right)_{2} \mathrm{CO}_{3}(288 \mathrm{mg}, 3.0 \mathrm{mmol})$ and water $(3 \mathrm{~mL})$ were added and the stirring was continued for $10 \mathrm{~min}$. The resulting mixture was poured into a narrow $(1 \mathrm{~cm}$ in diameter) column of silica gel (13 g). The organic portion was eluted with $10 \%$ ethanol in chloroform (190 mL). The yellow eluate was washed with saturated aqueous $\mathrm{NH}_{4} \mathrm{Cl}(50 \mathrm{~mL})$ and water $(50 \mathrm{~mL})$, dried $\left(\mathrm{Na}_{2} \mathrm{SO}_{4}\right)$, filtered, and evaporated to dryness. Trace amounts of DMF were removed by six subsequent co-destilations in vacuo at $50{ }^{\circ} \mathrm{C}$ with toluene $(30 \mathrm{~mL})$. The residue was dissolved in chloroform $(5 \mathrm{~mL})$ and chromatographed on silica gel (35 g) column using gradually $38 \%$ and $50 \%$ ethyl acetate in petroleum ether as mobile phase, affording product 10a (164 mg, $0.42 \mathrm{mmol}, 42 \%)$ as a white solid, m.p. $42-47{ }^{\circ} \mathrm{C} ; R_{\mathrm{f}}=0.21$ (38\% ethyl acetate in petroleum ether); ${ }^{1} \mathrm{H} \mathrm{NMR}(500 \mathrm{MHz}$, $\left.\mathrm{CDCl}_{3}\right) \delta 1.73(\mathrm{~s}, 3 \mathrm{H}), 5.20(\mathrm{~d}, 1 \mathrm{H}, J=15.6 \mathrm{~Hz}), 5.31(\mathrm{~d}, 1 \mathrm{H}, J=15.6 \mathrm{~Hz}), 5.45(\mathrm{~d}, 1 \mathrm{H}, J=14.8 \mathrm{~Hz}), 5.49(\mathrm{~d}$, $1 \mathrm{H}, J=14.8 \mathrm{~Hz}), 7.16-7.28(\mathrm{~m}, 3 \mathrm{H}), 7.32-7.39(\mathrm{~m}, 3 \mathrm{H}), 7.54(\mathrm{~s}, 1 \mathrm{H}), 7.67(\mathrm{ddd}, 1 \mathrm{H}, J=8.3,7.4,1.6 \mathrm{~Hz})$, $7.77(\mathrm{~d}, 1 \mathrm{H}, J=8.4 \mathrm{~Hz}), 7.96(\mathrm{dd}, 1 \mathrm{H}, J=7.7,1.5 \mathrm{~Hz}) ;{ }^{13} \mathrm{C} \mathrm{NMR}\left(126 \mathrm{MHz}, \mathrm{CDCl}_{3}\right) \delta$ 23.6, 39.1, 54.5, 70.6, 116.6, 119.5, 123.5, 124.3, 128.3, 128.8, 129.0, 129.3, 134.3, 137.1, 141.4, 143.0, 169.8, 191.3; IR (cm $\left.{ }^{-1}\right)$ : $v$ 3137, 3033, 2980, 2106, 1713, 1676, 1602, 1489, 1469, 1379, 1336, 1279, 1223, 765, 724; HRMS (ESI+): $m / z$ calcd for $\mathrm{C}_{20} \mathrm{H}_{18} \mathrm{~N}_{7} \mathrm{O}_{2}{ }^{+}[\mathrm{M}+\mathrm{H}]^{+} 388,1516$, found 388.1514. Anal. Calcd for $\mathrm{C}_{20} \mathrm{H}_{17} \mathrm{~N}_{7} \mathrm{O}_{2}$ (387.39): C, 62.01; H, 4.42; N, 25.31\%. Found: C, 61.74; H, 4.77; N, 25.15\%. 


\section{Conclusions}

We have developed a methodology for accessing bis(1,2,3-triazole) functionalized quinoline-2,4-diones in which the triazole heterocycles are present in substituents at positions 1 and 3 of the quinoline scaffold. Preliminary investigation has revealed that these compounds are potential multidentate ligands for arene-ruthenium.

Supplementary Materials: The following are available online: ${ }^{1} \mathrm{H}$ NMR and ${ }^{13} \mathrm{C}$ NMR spectra of all new compounds.

Author Contributions: D.M. performed the experiments. The manuscript was prepared through the contributions of R.K., M.G., D.U., S.K., and J.K.

Funding: The authors acknowledge the financial support from (internal grants No. IGA/FT/2018/007, funded from the resources of specific university research) and the Slovenian Research Agency (Research Core Funding Grant P1-0230, Project J1-8147, and Project J1-9166).

Acknowledgments: Tereza Dostálová and Lovro Kotnik participated in this study in their undergraduate projects. Their contributions are gratefully acknowledged.

Conflicts of Interest: The authors declare no conflict of interest.

\section{References}

1. Košmrlj, J. (Ed.) Click Triazoles, Topics in Heterocyclic Chemistry, 1st ed.; Springer: Berlin, Germany, 2012; Volume 28, ISBN 978-3-642-29429-7.

2. Crowley, J.D.; McMorran, D.A. 'Click-Triazole' coordination chemistry: Exploiting 1,4-disubstituted 1,2,3-Triazoles as ligands. In Topics in Heterocyclic Chemistry, 1st ed.; Košmrlj, J., Ed.; Springer: Berlin, Germany, 2012; Volume 28, pp. 31-83.

3. Schweinfurth, D.; Deibel, N.; Weisser, F.; Sarkar, B. Getting new ligands with a click. Nachr. Chem. 2011, 59, 937-941. [CrossRef]

4. Lee, S.; Flood, A.H. Binding Anions in Rigid and Reconfigurable Triazole Receptors. In Topics in Heterocyclic Chemistry, 1st ed.; Košmrlj, J., Ed.; Springer: Berlin, Germany, 2012; Volume 28, pp. 85-108.

5. Watkinson, M. Click Triazoles as Chemosensors. In Topics in Heterocyclic Chemistry, 1st ed.; Košmrlj, J., Ed.; Springer: Berlin, Germany, 2012; Volume 28, pp. 109-136.

6. Schweinfurth, D.; Hettmanczyk, L.; Suntrup, L.; Sarkar, B. Metal complexes of click-derived triazoles and mesoionic carbenes: Electron transfer, photochemistry, magnetic bistability, and catalysis. Z. Anorg. Allg. Chem. 2017, 643, 554-584. [CrossRef]

7. Tornøe, C.W.; Christensen, C.; Meldal, M. Peptidotriazoles on Solid Phase: [1-3]-Triazoles by Regiospecific Copper(I)-Catalyzed 1,3-Dipolar Cycloadditions of Terminal Alkynes to Azides. J. Org. Chem. 2002, 67, 3057-3064. [CrossRef] [PubMed]

8. Rostovtsev, V.V.; Green, L.G.; Fokin, V.V.; Sharpless, K.B. A Stepwise Huisgen Cycloaddition Process: Copper(I)-Catalyzed Regioselective "Ligation" of Azides and Terminal Alkynes. Angew. Chem. 2002, 114, 2708-2711. [CrossRef]

9. Kafka, S.; Hauke, S.; Salcinovic, A.; Soidinsalo, O.; Urankar, D.; Kosmrlj, J. Copper(I)-Catalyzed [3 + 2] Cycloaddition of 3-Azidoquinoline-2,4(1H,3H)-diones with Terminal Alkynes. Molecules 2011, 16, 4070-4081. [CrossRef]

10. Proisl, K.; Kafka, S.; Košmrlj, J. Chemistry and Applications of 4-Hydroxyquinolin-2-one and Quinoline-2,4-dionebased Compounds. Curr. Org. Chem. 2017, 21, 1949-1975. [CrossRef]

11. Bolje, A.; Urankar, D.; Košmrlj, J. Synthesis and NMR Analysis of 1,4-Disubstituted 1,2,3-Triazoles Tethered to Pyridine, Pyrimidine, and Pyrazine Rings. Eur. J. Org. Chem. 2014, 8167-8181. [CrossRef]

12. Bolje, A.; Košmrlj, J. A Selective Approach to Pyridine Appended 1,2,3-Triazolium Salts. Org. Lett. 2013, 15, 5084-5087. [CrossRef] [PubMed]

13. Perczel, A.; Atanasov, A.G.; Sklenář, V.; Nováček, J.; Papoušková, V.; Kadeřávek, P.; Žídek, L.; Kozłowski, H.; Watty, J; Hecel, A.; et al. The Eighth Central European Conference "Chemistry towards Biology": Snapshot. Molecules 2016, 21, 1381. [CrossRef] [PubMed] 
14. Kafka, S.; Proisl, K.; Kašpárková, V.; Urankar, D.; Kimmel, R.; Košmrlj, J. Oxidative ring opening of 3-hydroxyquinoline-2,4(1H,3H)-diones into $N$-( $\alpha$-ketoacyl)anthranilic acids. Tetrahedron 2013, 69, 10826-10835. [CrossRef]

15. Stadlbauer, W.; Laschober, R.; Lutschounig, H.; Schindler, G.; Kappe, T. Halogenation reactions in position 3 of quinoline-2,4-dione systems by electrophilic substitution and halogen exchange. Monatsh. Chem. 1992, 123, 617-636. [CrossRef]

16. Kafka, S.; Klásek, A.; Polis, J.; Košmrlj, J. Syntheses of 3-Aminoquinoline-2,4(1H,3H)-diones. Heterocycles 2002, 57, 1659-1682. [CrossRef]

17. Meldal, M.; Tornøe, C.W. Cu-Catalyzed Azide-Alkyne Cycloaddition. Chem. Rev. 2008, 108, $2952-3015$. [CrossRef] [PubMed]

18. Buckley, B.R.; Heaney, H. Mechanistic Investigations of Copper(I)-Catalysed Alkyne-Azide Cycloaddition Reactions. In Topics in Heterocyclic Chemistry, 1st ed.; Košmrlj, J., Ed.; Springer: Berlin, Germany, 2012; Volume 28, pp. 1-30.

19. Kimmel, R.; Kafka, S.; Košmrlj, J. Selective formation of glycosidic linkages of $N$-unsubstituted 4-hydroxyquinolin-2-(1H)-ones. Carbohydr. Res. 2010, 345, 768-779. [CrossRef] [PubMed]

20. Urankar, D.; Košmrlj, J. Concise and Diversity-Oriented Synthesis of Ligand Arm-Functionalized Azoamides. J. Comb. Chem. 2008, 10, 981-985. [CrossRef] [PubMed]

21. Iha, R.K.; Wooley, K.L.; Nyström, A.M.; Burke, D.J.; Kade, M.J.; Hawker, C.J. Applications of Orthogonal "Click" Chemistries in the Synthesis of Functional Soft Materials. Chem. Rev. 2009, 109, 5620-5686. [CrossRef] [PubMed]

22. Urankar, D.; Pinter, B.; Pevec, A.; De Proft, F.; Turel, I.; Košmrlj, J. Click-Triazole N2 Coordination to Transition-Metal Ions Is Assisted by a Pendant Pyridine Substituent. Inorg. Chem. 2010, 49, 4820-4829. [CrossRef] [PubMed]

23. Sá, M.M.; Ramos, M.D.; Fernandes, L. Fast and efficient preparation of Baylis-Hillman-derived (E)-allylic azides and related compounds in aqueous medium. Tetrahedron 2006, 62, 11652-11656. [CrossRef]

24. Boyer, J.H.; McCane, D.I.; McCarville, W.J.; Tweedie, A.T. Pyrido-2,3-furoxane. J. Am. Chem. Soc. 1953, 75, 5298-5300. [CrossRef]

Sample Availability: Samples of all compounds are available from the authors.

(C) 2018 by the authors. Licensee MDPI, Basel, Switzerland. This article is an open access article distributed under the terms and conditions of the Creative Commons Attribution (CC BY) license (http:/ / creativecommons.org/licenses/by/4.0/). 